\title{
Simple and Accurate Calibration of the Flory-Huggins Interaction Parameter
}

\author{
by \\ James Willis \\ A thesis \\ presented to the University of Waterloo \\ in fulfillment of the \\ thesis requirement for the degree of \\ Master of Science \\ in \\ Physics
}

Waterloo, Ontario, Canada, 2021

(C) James Willis 2021 


\section{Author's Declaration}

I hereby declare that I am the sole author of this thesis. This is a true copy of the thesis, including any required final revisions, as accepted by my examiners.

I understand that my thesis may be made electronically available to the public. 


\begin{abstract}
This work improves on a standard method used to calibrate $\chi$, the Flory-Huggins interaction parameter, in experimental systems. The common method is to fit the orderdisorder transition (ODT) of symmetric diblock copolymer melts to the mean-field prediction $(\chi N)_{O D T}=10.495$. This work improves the calibration by using the more accurate prediction of $(\chi N)_{O D T}$ from Morse and coworkers, correcting for the small degrees of polydispersity and compositional asymmetry that exist in real diblock copolymers. To find the correction, polydisperse lattice simulations are conducted over a wide range of invariant polymerization indices. The correction for compositional asymmetry is extracted from simulations for asymmetric diblocks conducted by Ghasimakbari and Morse. This improved calibration is demonstrated for 19 different chemistries, using previously published data from experiments. This calibration provides a considerable increase in accuracy, while still being simple to apply.
\end{abstract}




\section{Acknowledgements}

I want to thank my supervisor, Prof. Mark Matsen, for his support and guidance. Also, I am grateful to my graduate committee Prof. Robert Wickham and Prof. Jamie Forrest for their support. Special thanks to Dr. Thomas Beardsley for his input and expertise in this area. Computer resources were provided by Compute Canada. 


\section{Table of Contents}

List of Figures vii

List of Tables $\quad$ x

List of Abbreviations $\quad$ xii

List of symbols $\quad$ xiii

1 Introduction $\quad 1$

1.1 Polymer Melts . . . . . . . . . . . . . . . . . . 1

1.2 Block Copolymers . . . . . . . . . . . . . . . . . . 6

1.3 Gaussian chain . . . . . . . . . . . . . . . . . 9

1.4 Mean-Field Theory . . . . . . . . . . . . . . . . . . . 11

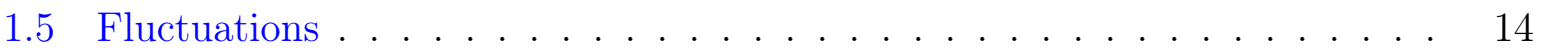

1.6 Universality . . . . . . . . . . . . . . . . . . 16

2 Monte Carlo Simulations $\quad 19$

2.1 Lattice Model . . . . . . . . . . . . . . . . . . . . . . 19

2.2 Statistical Mechanics . . . . . . . . . . . . . . . . . . 21

2.3 Monte Carlo Steps . . . . . . . . . . . . . . . . . . . . . . 22

2.4 Parallel Tempering . . . . . . . . . . . . . . . . . . 27 
3 Calibration of the Lattice Model 30

3.1 Calibration for Statistical Segment Length . . . . . . . . . . . . . . 30

3.2 Calibration of $\chi \ldots \ldots \ldots$. . . . . . . . . . . . . . . . . . . . . . . . .

4 Order-Disorder Transition 44

4.1 Monodisperse Order-Disorder Transition . . . . . . . . . . . . . . . . . 41

4.2 Polydisperse Correction to the ODT . . . . . . . . . . . . . . 43

4.3 Compositional Asymmetry Correction to the ODT . . . . . . . . . . 49

5 Calibration of Experimental Systems 53

6 Conclusion $\quad 63$

6.1 Conclusion . . . . . . . . . . . . . . . . . . 63

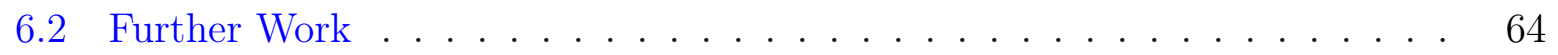

$\begin{array}{ll}\text { Bibliography } & 65\end{array}$

$\begin{array}{ll}\text { Appendices } & 73\end{array}$

$\begin{array}{ll}\text { A Calibration Data } & 74\end{array}$

B Random Phase Approximation $\quad 95$ 


\section{List of Figures}

1.1 Diagram of various polymer architectures. . . . . . . . . . . . . 2

1.2 Diagram of a polymer showing the points $\vec{r}\left(s_{1}\right)$ and $\vec{r}\left(s_{2}\right) \ldots \ldots$

1.3 Diagram of a polymer showing the end-to-end distance $\vec{R}_{0}$. . . . . . . . 4

1.4 Minority domains from the periodically ordered phases observed in diblock copolymer melts. They are, lamellar (L), gyroid $(\mathrm{G})$, cylindrical $(\mathrm{C})$, and spherical $(\mathrm{S}) . \ldots \ldots \ldots . \ldots \ldots$

1.5 Diblock copolymer phase diagram reproduced from Matsen and Bates [13]. 14

1.6 Diblock copolymer phase diagram reproduced from Fredrickson-Helfand 1989 [15]. . . . . . . . . . . . . . . . . . . . 15

1.7 Comparison of the disorder state structure functions for the three different

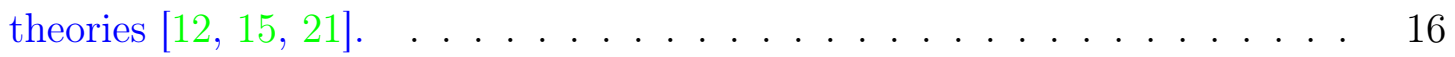

1.8 Plot of the ODT of symmetric diblocks over a range of $\bar{N}$. [18]. Different symbols are from different models that where calibrated independently. .

2.1 Example of the slithering snake move. For simplicity, the move is demonstrated on a two-dimensional simple square lattice . . . . . . . . . . .

2.2 Example of the crankshaft move. For simplicity, the move is demonstrated on a two-dimensional simple square lattice . . . . . . . . . . . . . . . .

2.3 Example of the head-to-tail flip move. For simplicity, the move is demonstrated on a two-dimensional simple square lattice . . . . . . . . . . . . .

2.4 Example of the polymer rebridging move. For simplicity, the move is demonstrated on a two-dimensional simple square lattice. The sites with two monomers on them are marked with the number $2 . . . . . . . . .$. 
3.1 Calibration of the statistical segment length for $\mathrm{c}=1,2,3$, and 5 . The symbols are from the lattice simulations and the lines are the fits. . . . . .

3.2 Fits of the number of intermolecular contacts used to determine $z_{\infty}$ for $c=$ $1,2,3$, and 5 . The symbols are from the lattice simulations and the lines are the fits. . . . . . . . . . . . . . . . .

3.3 Leibler function fits for $N=30$ with $c=2$ and $5 \ldots \ldots \ldots$

3.4 Peaks in structure functions for $c=1$. Symbols are from the lattice simulations and curves are ROL predictions. The fit to ROL predictions for $\bar{N}=$ $135,180,271$, and 406 give values of $C_{2}=33.6$ and $C_{3}=-74 \ldots \ldots$

3.5 Peaks in structure functions for $c=2$. Symbols are from the lattice simulations and curves are ROL predictions. The fit to ROL predictions for $\bar{N}=$ 372,496744 , and 1240 give values of $C_{2}=208$ and $C_{3}=-63 \ldots \ldots$.

3.6 Peaks in structure functions for $c=3$. Symbols are from the lattice simulations and curves are ROL predictions. The fit to ROL predictions for $\bar{N}=$ $678,968,1452$, and 2420 give values of $C_{2}=0.68$ and $C_{3}=-0.17 \ldots \ldots$.

3.7 Peaks in structure functions for $c=5$. Symbols are from the lattice simulations and curves are ROL predictions. The fit to ROL predictions for $\bar{N}=5990,3594,2396$, and 1797 give values of $C_{2}=4.15$ and $C_{3}=-8.5$.

4.1 Average number of $\mathrm{AB}$ contacts, $\left\langle n_{A B}\right\rangle$, from parallel-tempering simulations of monodisperse diblock copolymers with $N=50$ and with $c=1,2,3$, and 5 monomers per site, respectively. Open symbols are started from a lamellar configuration and closed symbols are started from disorder. the box size $L$ is picked so that there are approximately 3 periods in the box. . . . . . . .

4.2 Location of the ODT, $(\chi N)_{O D T}$, as a function of the invariant polymerization index, $\bar{N}$. The curve is the universal curve, Equation 4.1, and the points are the results from our lattice simulation with $N=50$ and $f=0.5$. The uncertainty from the lattice model predictions are the error bars. . . . . . .

4.3 Metastability loops in the average number of intermolecular contacts per monomer $\left\langle n_{A B}\right\rangle / M$ over a range of the universal interaction strengths, $\chi N$. The melts are polydisperse with index $Đ$, average chain length $\langle N\rangle=50$ and $c=1,2,3$, and 5 monomers per site respectively. Open symbols are started from a lamellar configuration and closed symbols are started from disorder. . . . . . . . . . . . . . . . . . . . . 
4.4 Fits of the polydispersity correction used to find $\kappa$. Symbols are data from the lattice simulations. The error bars are the widths of the metastability loops and the lines are the fits to Equation 4.2. . . . . . . . . . . 46

4.5 Fitting Equation 4.2 to the prediction of $(\chi N)_{O D T}$ from RPA gives the value of $\kappa$ for $\bar{N}=\infty$ as $\kappa=-0.77 \ldots \ldots \ldots \ldots$

4.6 Dependence of the linear correction for polydispersity, $\kappa$, on the invariant polymerization index, $\bar{N}$. Closed circles are from our simulation data, the open circle is from mean-field theory, and the line is Equation 4.7 with $B=-0.49$ and $C=17.5$. . . . . . . . . . . . . . . . .

4.7 ODTs for different values of compositional asymmetry. Points are from the data in Table 4.3, and lines are fits to Equation 4.8. The parameter $\gamma$ is given by the slope of the lines. . . . . . . . . . . . . . . . . .

4.8 Dependence of the linear correction for polydispersity on the invariant polymerization index $\bar{N}$. Closed symbols are from the Morse group data [31], while the open symbol is a mean-field value from Matsen and Bates [11] . .

5.1 Plot of the calibration of $\chi$ for PCHE-PMMA using data from Kennemur, Bates, and Hillmyer [59]. The calibration based on mean field ODT Equation 5.8 gives $A=138$ and $B=0.151$. The calibration based on Fredrickson Helfand ODT Equation 5.6 gives $A=275$ and $B=0.325$. The calibration based on Universal curve ODT Equation 5.7 gives $A=429$ and $B=0.536$. The calibration based on this works correction ODT Equation 5.8 gives $A=543$ and $B=0.707 \ldots \ldots . \ldots \ldots$

5.4 Analogous plots to Figure 5.1 for 19 different experimental systems. Calibration of $\chi$ for 19 experimental systems. . . . . . . . . . . . . . 


\section{List of Tables}

$3.1 c$ is maximum the number of monomers per lattice site, $a / b$ is the statistical segment length relative to the bond length. $\rho_{0}$ is the monomer density, and $\bar{N} / N$ is the ratio of invariant polymerization index to contour length $N$. . 32

$3.2 z_{\infty}$ values for the four models with different numbers of monomers per site, c. 34

3.3 A list of all the parameters for the simulations used to calibrate $\chi(\alpha)$. . 36

3.4 Parameters for Equation 3.5 relating $\chi$ and $\alpha$, calculated by a fit of the Monte Carlo simulation data to predictions from ROL theory. . . . . . . .

$4.1(\chi N)_{O D T}$ for melts with three different polydispersities of $\mathrm{Ð}=1,1.05$, and 1.10 for our models with $c=1,2,3$, and 5 monomers per site. . . . . . . .

4.2 Linear polydispersity correction, $\kappa$, for the four models with different numbers of monomers per site, c. . . . . . . . . . . . . . . . 47

4.3 ODTs for $\bar{N}=480$ and 1920 and various compositions $f$, with $Đ=1$ from Ghasimakbari, T.; Morse, [31]. . . . . . . . . . . . . .

5.1 Molecular data used in calculating the number of segments and the segment mass. $R_{0}^{2} / M_{n}$ is the literature value of how radius of gyration scales with chain length. $\rho$ is the mass density from the literature and $a$ is the statistical segment length calculated from Equation 5.3. . . . . . . . . . .

5.2 Tabulation of data for PCHE-PMMA for degree of polymerization $N$ calculated with Equation 5.1, polydispersity Đ, composition $f$, and the experimental temperance of the order-disorder transition $T_{O D T}$ from [59]. . . . .

5.3 Tabulation of calibrated $\chi$ values for data from various experimental papers. $\mathrm{A}$ and $\mathrm{B}$ are the fitting parameters from Equation $5.4 . \ldots . . .$. 
A.1 Molecular data at $T=140^{\circ} \mathrm{C}$ used in calculating the number of segments and the segment mass. $R_{0}^{2} / M_{N}$ is how the radius of gyration scales with chain length from the literature. $\rho$ is the density from the literature. $a$ is the calculated statistical segment length. . . . . . . . . . . . 


\section{List of Abbreviations}

- ROL is renormalized one-loop.

- RPA is the random phase approximation.

- SCFT is self consistent field theory.

- ODT is the order-disorder transition.

- GCM is the Gaussian chain model. 


\section{List of symbols}

- $N$ : Degree of polymerization

- $S$ : Entropy

- $T$ : Temperature

- $k_{B}$ : Boltzmann's constant

- $\rho_{0}$ : Polymer segment density

- $b$ : Fixed bond length

- $s$ : Chain contour parameter

- $\chi$ : Flory-Huggins polymer-polymer interaction parameter

- $F$ : Free energy

- $Z$ : Partition function

- $Q$ : Single-chain partition function

- $q$ : Chain propagator

- $q^{\dagger}$ : Reverse chain propagator

- $\phi$ : Polymer concentration

- $\phi_{A}$ : Polymer concentration of A segments

- $\phi_{B}$ : Polymer concentration of B segments

- $R_{0}$ : Mean square end-to-end distance

- $R_{g}$ : Radius of gyration

- $w_{A}$ : Mean-field interactions from A segments

- $w_{B}$ : Mean-field interactions from B segments

- $U_{B}$ : The single bond energy 
- $a$ : Statistical segment length

- $\Phi$ : Single-chain correlation function

- $N_{n}$ : Number-averaged molecular weight

- $N_{n w}$ : Weight-averaged molecular weight

- Đ: Polydispersity index

- $f$ : The composition of A segments in the melt

- $\gamma_{A B}$ : The ratio of statistical segment lengths

- $\epsilon_{A A}$ : Interaction between $\mathrm{A}$ and $\mathrm{A}$ segments

- $\epsilon_{A B}$ : Interaction between A and B segments

- $\epsilon_{B B}$ : Interaction between $\mathrm{B}$ and $\mathrm{B}$ segments

- $s_{i, j}$ : The RPA tensor

- $\bar{N}$ : Invariant polymerization index

- $V$ : Volume of the simulation box

- $L$ : Number of lattice points along the side of the lattice

- $M$ : Number of polymer segments on the lattice

- $c$ : Maximum number of monomers per lattice point

- $\epsilon$ : Interaction energy per contact on the lattice

- $\alpha$ : Dimensionless interaction parameter on the lattice

- $N_{A B}$ : Number of A-B contacts on the lattice

- $R_{[0,1]}$ : Random number on the range 0 to 1

- $z$ : The number of intermolecular contacts

- $z_{\infty}$ : The average number of intermolecular contacts of an athermal melt with infinity long chains.

- $\kappa$ : The linear correction factor for polydispersity

- $\gamma$ : Correction for compositional asymmetry 


\section{Chapter 1}

\section{Introduction}

\section{$1.1 \quad$ Polymer Melts}

A polymer is a high molecular weight molecule that is created by repeatedly joining together a large number of small molecules, called monomers. Most polymers are flexible, despite the fact that the carbon-carbon bond angle between monomers is fixed. Rotations around the carbon-carbon bonds give the chain a large number of configurations [1]. Therefore, the chain is flexible because the number of allowed configurations of the monomers between two points on the chain is large and this allows two monomers separated by a sufficient number of bonds to have any relative angle between them.

There are a large number of different polymer architectures. The simplest architecture is linear polymers, which are polymers where the monomers from chains without any branches. Another type of polymer is star polymers. These polymers have a central junction where a number of different arms leave from. There is a large number of different architectures including ring, comb, brush, and dendritic polymers, some of which are illustrated in Figure 1.1. However, this work will only involve linear polymers. 


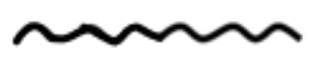

Linear polymer

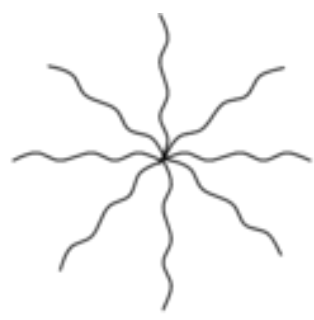

Star polymer

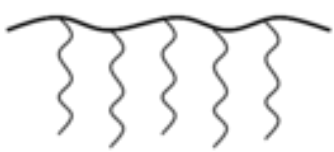

Comb polymer

Figure 1.1: Diagram of various polymer architectures.

Polymers can be part of different types of systems. Polymer solutions are a mix of polymers with a solvent. The interaction between the polymers and the solvent control the configurations of the chains in the solution. If there is an attractive interaction between the polymers and the solvent, then the polymer will take a extended configuration, so that it contact more solvent molecules. If the interaction is repulsive, then the polymers will take a more compact configuration. Another type of polymer system is a polymer melt. Melts are a dense system that have only polymers and no solvent. Thus the only important interaction are the interactions is between polymers. This work only involves polymer melts.

With this we can coarse-grain the polymer into a chain of $N$ independent segments, each of volume $\rho_{0}^{-1}$. These segments are defined such that they contain a large enough number of monomers for the bond angles between the segments to have any relative angle. As such, the polymer can be modelled, as a series of beads connected by springs that represent the configurational entropy of the monomers between the beads. 


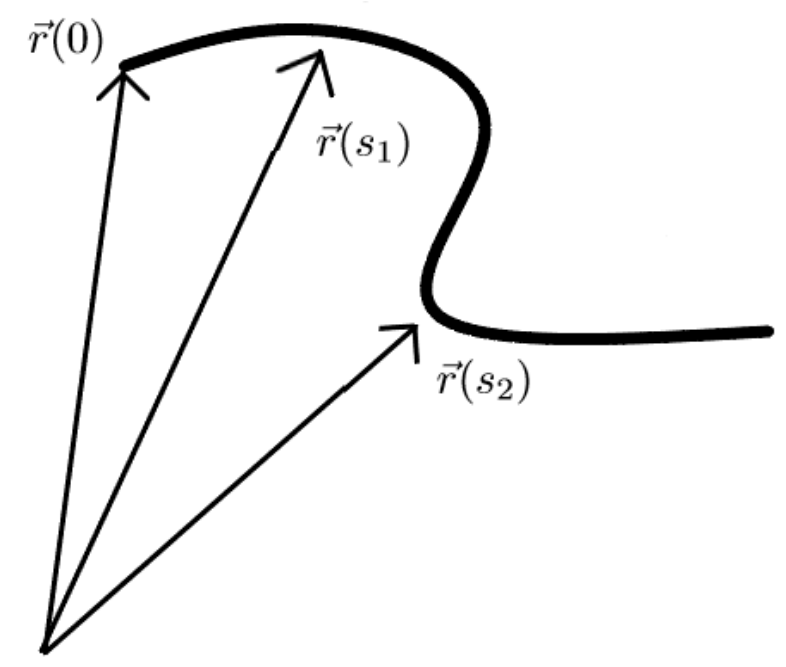

Figure 1.2: Diagram of a polymer showing the points $\vec{r}\left(s_{1}\right)$ and $\vec{r}\left(s_{2}\right)$.

The strength of the entropic spring can be found by fixing two points on the chain, $s_{1}$ and $s_{2}$, a distance of $R=\left|\vec{r}\left(s_{1}\right)-\vec{r}\left(s_{2}\right)\right|$ apart. The number of combinations of bonds is called $\Omega$, the logarithm of which gives the entropy $S=k_{B} \ln (\Omega)$. This gives a bond potential $U_{B}(R)=-T S$ which has a minimum at $R=0$, because the chain is minimally stretched and so has the most possible configurations. With this knowledge, the potential can be Taylor expanded around $R=0$. Because the zeroth-order term is a constant, it contributes only an energy proportional to the total number of bonds and thus it can be ignored. The first-order term is odd and so must be zero for the stretching energy to be symmetric around $R=0$. Therefore, the first non-trival term is the quadratic $R^{2}$ term. Therefore, the stretching energy of a segment is

$$
U_{B}(R)=\frac{3}{2 \beta a^{2}} R^{2}
$$

where $\beta=\frac{1}{k_{B} T}$ is the thermodynamic parameter and $a$ is the average length of the segment called the statistical segment length. This can be shown by finding the thermodynamic average of the bond length squared

$$
\left\langle|\vec{R}|^{2}\right\rangle=\frac{\int|\vec{R}|^{2} e^{-\beta U_{B}(|\vec{R}|)} d^{3} \vec{R}}{\int e^{-\beta U_{B}(|\vec{R}|)} d^{3} \vec{R}}=\frac{4 \pi \int r^{2} e^{\frac{-3}{2 a^{2}} r^{2}} d r}{4 \pi \int e^{\frac{-3}{2 a^{2}} r^{2}} d r}=a^{2} .
$$

Taking the square root of Equation 1.2 gives a root-mean-squared average segment length of $a$, which is why $a$ is referred to as the statistical segment length. Repeating this for the 
$\mathrm{N}$ bonds in the polymer, we get the root-mean-squared end-to-end distance of

$$
R_{0}^{2}=\left\langle\left|\sum_{n=1}^{N} \vec{R}_{n} \cdot \sum_{m=1}^{N} \vec{R}_{m}\right|\right\rangle=\sum_{n=1}^{N} \sum_{m=1}^{N}\left\langle\left|\vec{R}_{n} \cdot \vec{R}_{m}\right|\right\rangle .
$$

Since the bonds with $n \neq m$ are uncorrelated by construction, it follows that $\left\langle\vec{R}_{n} \cdot \vec{R}_{m}\right\rangle=$ $\delta_{n, m}\left\langle\left|\vec{R}_{n}\right|^{2}\right\rangle=\delta_{n, m} a^{2}$. Putting this into the sum gives

$$
R_{0}^{2}=\sum_{n=1}^{N} \sum_{m=1}^{N} \delta_{n, m} a^{2}=\sum_{n=1}^{N} a^{2}=N a^{2} .
$$

This shows that the characteristic size of a polymer is $R_{0}=a N^{\frac{1}{2}}$.

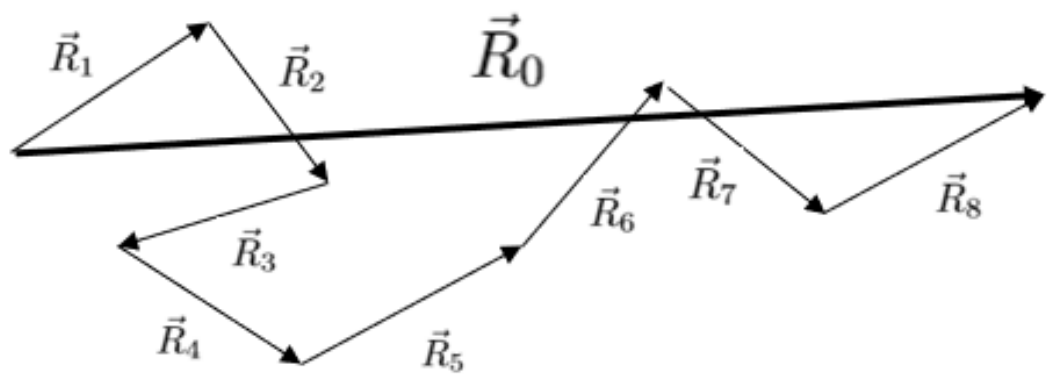

Figure 1.3: Diagram of a polymer showing the end-to-end distance $\vec{R}_{0}$.

Another, more general measure of polymer size is the radius of gyration $R_{g}$, defined by

$$
R_{g}^{2}=\frac{1}{N} \sum_{n=1}^{N}\left\langle\left(\vec{R}_{n}-\vec{R}_{c m}\right)^{2}\right\rangle=\frac{1}{N} \sum_{n=1}^{N}\left\langle\left(\left|\vec{R}_{n}\right|^{2}-2 \vec{R}_{n} \cdot \vec{R}_{c m}+\left|\vec{R}_{c m}\right|^{2}\right)\right\rangle
$$

where $\vec{R}_{c m}=\frac{1}{N} \sum_{n=1}^{N} \vec{R}_{n}$ is the center of mass of the polymer. Expanding and simplifying gives

$$
R_{g}^{2}=\frac{1}{N^{2}} \sum_{n=1}^{N} \sum_{m=1}^{N}\left\langle\left(\left|\vec{R}_{n}\right|^{2}-\vec{R}_{n} \cdot \vec{R}_{m}\right)\right\rangle=\frac{1}{N^{2}} \sum_{n=1}^{N} \sum_{m=1}^{N}\left\langle\left|\vec{R}_{n}-\vec{R}_{m}\right|^{2}\right\rangle .
$$

Taking the average of the radius of gyration and substituting $\left\langle\left|\vec{R}_{n}-\vec{R}_{m}\right|^{2}\right\rangle=|n-m| a^{2}$ for the end-to-end length of a polymer with $n-m$ monomers, simplifies Equation 1.6 to the 
following:

$$
R_{g}^{2}=\frac{1}{N^{2}} \sum_{n=1}^{N} \sum_{m=1}^{N}\left\langle\left|\left(\vec{R}_{n}-\left.\vec{R}_{m}\right|^{2}\right\rangle=\frac{a^{2}}{N^{2}} \sum_{n=1}^{N} \sum_{m=1}^{N}\right| n-m\right|=\frac{a^{2} N}{6}
$$

However, this analysis only works if the polymer segments are all independent of each other. In real polymers there is an excluded-volume interaction between the segments that acts to swell the size of the polymer. These interactions come from the inability of the molecules that make up the monomers to occupy the same physical space. These interactions prevent chains from crossing themselves, and thus the path of the chain follows a self-avoiding random walk.

In dense polymer systems like polymer melts, there are a large number of polymers, $n$. The same excluded-volume interactions that exists within a single polymer chain also exist between different chains in the melts. These intermolecular interactions between different polymers in the system affect the trajectories of the polymers in the melt such that they no longer follow self-avoiding random walks. The averaged excluded-volume interactions are dictated by the density of the monomers. In our single-chain example, the density of monomers is higher near the center of mass of the polymer and so this force acts to push the monomers in the chain away from the center. This is the force that expands the polymer and makes it follow a self-avoiding random walk.

A dense polymer melt is incomprehensible, meaning that the monomer density is constant. Because the individual monomers in a chain do not experience a difference between forces from monomers on the same chain and forces from other polymers, the incompressibility of the melt means there is no difference in the density to cause the force that makes the polymer follow a self-avoiding random walk. This implies that the interactions between polymers in the melt act to screen the polymer self interactions [1]. With this screening of the excluded-volume interactions, the polymers no longer follow a self-avoiding random walk but rather a simple random walk of size $R_{0}=a N^{\frac{1}{2}}$. Hence, the chains are, once again, ideal.

So far, we have assumed that all the polymers in the melt have the same length, $N$. This is referred to as a monodisperse melt. In real polymer melts, the synthesis processes leads to a distribution of polymer lengths [3], $N_{i}$, known as polydisperse melts. The number of polymers that have that length $N_{i}$, is called $n_{i}$. This enables us to define a probability distribution of molecular weights,

$$
p\left(N_{i}\right)=\frac{n_{i}}{\sum_{j=0}^{\infty} n_{j}}
$$


The average number of segments in a chain can be represented with two quantities: the number-average chain length,

$$
N_{n}=\sum_{i=1}^{n} N_{i} p\left(N_{i}\right)=\frac{\sum_{i=1}^{n} N_{i} n_{i}}{\sum_{i=1}^{n} n_{i}},
$$

and the weight-average chain length

$$
N_{w}=\frac{\sum_{i=1}^{n} n_{i} N_{i}^{2}}{\sum_{i=1}^{n} n_{i} N_{i}} .
$$

The width of the probability distribution can be represented by the polydispersity index. This index is defined as the ratio of the weight-average chain length and the number-average chain length

$$
\mathrm{Ð}=\frac{N_{w}}{N_{n}}
$$

The polydispersity index is related to the breadth of the molecular weight distribution,

It is common in the polymer literature to use the Schultz-Zimm distribution [5, 6, 7] defined as

$$
p(N)=\frac{K^{K} N^{K-1}}{N_{n}^{K} \Gamma(K)} e^{-\frac{K N}{N_{n}}},
$$

where the polydispersity index is related to $K$ by $Đ=1+\frac{1}{K}$. However, in practice the molecular weight distribution can have many different shapes depending on the particular details of the chemical procedure used to synthesize the polymer melt. The overall shape of the molecular weight distribution will affect the behavior of the polymer melt [8, 9], but due to the low polydispersity of melts considered in this work, only the lowest moments of the distribution have an effect on the behavior of the system [10]. Since any system that has a low polydispersity index can be well approximated by any distribution with the same polydispersity index, this work will use the Schultz-Zimm distribution.

\subsection{Block Copolymers}

Polymers that are made of multiple types of monomers with different chemistries are called copolymers. There are many different types of copolymers like random copolymers, where the different monomers are placed randomly along the chain, and block copolymers, where 
the polymer is made of distinct blocks with different chemical structure. The simplest type of block copolymer is the diblock copolymer, where there are two different blocks of monomers joined together at a junction, called $A$ and $B$ type blocks.

Doing the coarse-graining from the previous section on each side of the chain, one side of the chain has $N_{A}$ segments of length $a_{A}$ and the other has $N_{B}$ segments of length $a_{B}$. We can define the total number of segments $N=N_{A}+N_{B}$ and the composition $f=N_{A} / N$. Another parameter that can affect the chain is $\gamma_{A B}=a_{A} / a_{B}$, the ratio of statistical segment lengths. Hence, the chain can be described by the parameters $\left(N, f, \gamma_{A B}\right)$. Because the $A$ and $B$ sides of the chain are chemically distinct, there are important interactions between them. We can write the energy of the interactions in terms of three independent interaction parameters, $\epsilon_{A A}, \epsilon_{A B}$, and $\epsilon_{B B}$ that control the strength of the interactions. This gives the interaction energy as

$$
\begin{aligned}
u_{\alpha \beta}= & \epsilon_{A A} \int_{0}^{f} \int_{0}^{f} d s d t \delta\left(\vec{r}_{\alpha}(s)-\vec{r}_{\beta}(t)\right)+ \\
& 2 \epsilon_{A B} \int_{0}^{f} \int_{f}^{1} d s d t \delta\left(\vec{r}_{\alpha}(s)-\vec{r}_{\beta}(t)\right)+ \\
& \epsilon_{B B} \int_{f}^{1} \int_{f}^{1} d s d t \delta\left(\vec{r}_{\alpha}(s)-\vec{r}_{\beta}(t)\right) .
\end{aligned}
$$

Now we need to simplify the above by substituting the definitions of monomer density:

$$
\phi_{A}(\vec{r})=\frac{N}{\rho_{0}} \sum_{\alpha=1}^{n} \int_{0}^{f} d s \delta\left(\vec{r}-\vec{r}_{\alpha}(s)\right)
$$

and

$$
\phi_{B}(\vec{r})=\frac{N}{\rho_{0}} \sum_{\alpha=1}^{n} \int_{f}^{1} d s \delta\left(\vec{r}-\vec{r}_{\alpha}(s)\right)
$$

into the expression for $u_{\alpha \beta}$. Taking the sum over all $\alpha$ and $\beta$ to get the total energy and applying the delta function identity for $\delta\left(\vec{r}_{\alpha}(s)-\vec{r}_{\beta}(t)\right)$, we can simplify to get an energy functional in terms of the density,

$$
U=\frac{\rho_{0}^{2}}{2 N^{2}} \sum_{\alpha=1}^{n} \sum_{\beta=1}^{n} u_{\alpha \beta}=\frac{1}{2} \int_{\mathbb{R}^{3}} d^{3} \vec{r}\left[\epsilon_{A A} \phi_{A}(\vec{r}) \phi_{A}(\vec{r})+2 \epsilon_{A B} \phi_{A}(\vec{r}) \phi_{B}(\vec{r})+\epsilon_{B B} \phi_{B}(\vec{r}) \phi_{B}(\vec{r})\right]
$$


Inserting the incompressibility condition, $\phi_{A}(\vec{r})+\phi_{B}(\vec{r})=1$, into the above Equation 1.26 to remove the dependency on $\phi_{B}(\vec{r})$ from Equation 1.26 , we obtain

$$
U=\frac{\left(\epsilon_{A A}-2 \epsilon_{A B}+\epsilon_{B B}\right)}{2} \int_{\mathbb{R}^{3}} d^{3} \vec{r} \phi_{A}(\vec{r})\left(1-\phi_{A}(\vec{r})\right) .
$$

Making the definition $\chi=\frac{\left(\epsilon_{A A}-2 \epsilon_{A B}+\epsilon_{B B}\right)}{2 k_{B} T \rho_{0}}$ simplifies Equation 1.27 to

$$
U=k_{B} T \chi \rho_{0} \int_{\mathbb{R}^{3}} d^{3} \vec{r}\left[\phi_{A}(\vec{r})\left(1-\phi_{A}(\vec{r})\right)\right]
$$

From this, we can see all the independent interaction parameters have been put into a single parameter $\chi$ that affects the polymer melt.

With this, we now have a total of four parameters $\left(\chi, N, f, \gamma_{A B}\right)$ that affect the diblock copolymer melt. This model is called the standard Gaussian chain model. It is widely used in theoretical work due to its strong track record of making predictions [2, 11, 3, 4] and its relative simplicity.

Diblock copolymers form a variety of different phases, because the $\chi$ parameter is usually positive, giving a positive energy contribution to contacts of unlike blocks. That means, when $\chi$ is high, the $\mathrm{A}$ and $\mathrm{B}$ type segments form distinct regions in order to minimize the number of unfavorable contacts and thus the energy of the system. If the blocks of the copolymer were not joined together at the junction, they would fully phase separate and form two distinct phases: an A-rich phase and a B-rich phase. However, in diblocks this is impossible, so the segregation happens on scales comparable to the polymer size, $R_{0}$. This forms microstructures with the junctions of the polymers on the interfaces between the $\mathrm{A}$ and $\mathrm{B}$ regions. The simplest of these structures is the lamellar phase $(L)$ formed when $f \approx 0.5$. The lamellar phase consists of alternating sheets rich in $\mathrm{A}$ and B monomers (see Figure 1.4). Other simple phases predicted by mean-field theory are hexagonal-packed cylinders $(C)$ and body-centered-cubic spheres $(S)$, both composed of the minority component of the melt. There is also a complex phase window with network phases including gyroid $(G)$ and the Fddd phase $O^{70}$. 


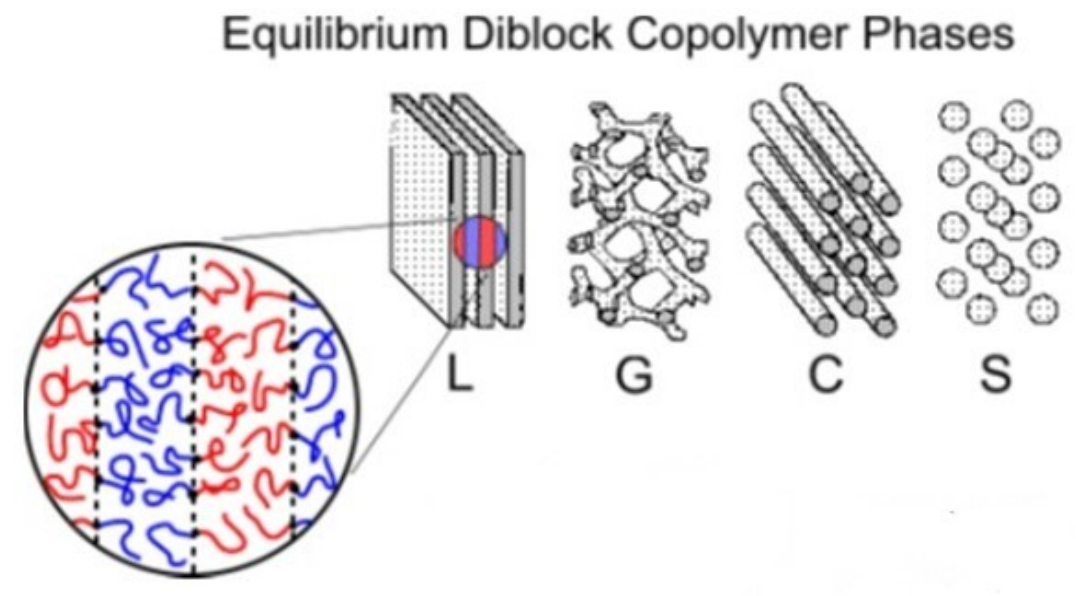

Figure 1.4: Minority domains from the periodically ordered phases observed in diblock copolymer melts. They are, lamellar (L), gyroid (G), cylindrical (C), and spherical (S).

\subsection{Gaussian chain}

To analyze this random walk, consider adding one bead to the end of the chain of length $s$ at point $\vec{r}_{s}$. The probability that the bead is at a position $\vec{r}_{s+1}$ is given by the Boltzmann weight

$$
\Phi\left(\vec{r}_{s}, \vec{r}_{s+1}\right)=\frac{1}{Z_{B}} e^{\frac{-3}{2 a^{2}}\left|\vec{r}_{s}-\vec{r}_{s+1}\right|^{2}}
$$

The normalization constant $Z_{B}$ can be found by forcing $\int_{\mathbb{R}^{3}} \Phi\left(\vec{r}_{s}, \vec{r}_{s+1}\right) d^{3} \overrightarrow{r_{s}}=1$. The total probability of the chain can be found by adding a monomer to the end of the chain $\mathrm{N}$ times. This grows the chain from length 1 to length $\mathrm{N}$. The probability distribution of a monomer being added to a chain of length $s$ is given by

$$
P_{s}(\vec{r})=\int_{\mathbb{R}^{3}} \Phi\left(\overrightarrow{r^{\prime}}, \vec{r}\right) P_{s-1}\left(\overrightarrow{r^{\prime}}\right) d^{3} \overrightarrow{r^{\prime}}
$$

This gives a recurrence relationship that can be solved by a Fourier transform to give

$$
\hat{P}_{s}(\vec{k})=\left[e^{-\frac{a^{2} k^{2}}{6}}\right]^{s} \hat{P}_{0}(\vec{k})
$$


For simplicity, we fix the starting end of the polymer at position $\vec{r}_{0}=0$. This corresponds to the starting condition $\hat{P}_{0}(\vec{k})=1$, which comes from the fact that the end is localized to a delta function $P_{0}(\vec{r})=\delta(\vec{r})$. Inverting the Fourier transform gives the value of $P_{N}(\vec{r})$ as

$$
P_{s}(\vec{r})=\frac{3}{2 \pi s a^{2}} e^{-3 \frac{|\vec{r}|^{2}}{2 s a^{2}}}
$$

This shows that the probability distribution of the monomers in the chain is Gaussian. We can see that as we move down the chain from $s=1$ to $s=N$, the variance of the probability distribution of the monomer increases. However, as the number of monomers is increased, the distribution is still Gaussian. This leads to the idea of taking a limit as the number of monomers in the chain goes to infinity. In this limit, the model is called the continuous Gaussian chain model.

The continuous Gaussian chain model is commonly used for analytical calculations $[2,4,3]$. In the limit as the number of segments $N$ in the chain gets large, while keeping the chain size, $R_{0}$, constant, the discrete segments of the chain can be replaced by a single continuous parameter $s$ in a range $[0, N]$. This model is called the continuous Gaussian chain model because the two-point correlation function along the chain is Gaussian.

To calculate the partition function for the Gaussian chain model, the chain propagator $q(\vec{r}, s)$ is used. It tells you the probability of finding the $s^{t h}$ segment of the chain at point $\vec{r}$. We simply replace $N$ in the recurrence relationship with the continuous parameter $s$, $P_{N}(\vec{r})$ by $q(\vec{r}, s)$ and substitute in $\overrightarrow{r^{\prime}}=\vec{r}+\Delta \vec{r}$ to get

$$
q(\vec{r}, s+\Delta s)=\int_{\mathbb{R}^{3}} \Phi(\vec{r}+\Delta \vec{r}, \vec{r}) q(\vec{r}+\Delta \vec{r}, s) d^{3} \Delta \vec{r} .
$$

We can simplify the above Equation 1.12 by Taylor expanding around $s$ and $\vec{r}$. This works because as the number of segments gets large, the space between the segments $\Delta \vec{r}$ and $\Delta s$ gets small, making the Taylor expansion

$q(\vec{r}, s)+\frac{d q(\vec{r}, s)}{d s} \Delta s=\int_{\mathbb{R}^{3}} \Phi(\vec{r}+\Delta \vec{r}, \vec{r})\left(q(\vec{r}, s)+\nabla q(\vec{r}, s) \cdot \Delta \vec{r}+\frac{1}{2} \sum_{i=1}^{3} \sum_{j=1}^{3} \frac{\partial^{2} q(\vec{r}, s)}{\partial r_{i} \partial r_{i}} \Delta r_{i} \Delta r_{j}\right) d^{3} \Delta \vec{r}$

accurate. Expanding and substituting $\langle f(\vec{r})\rangle=\int_{\mathbb{R}^{3}} \Phi(\vec{r}+\Delta \vec{r}, \vec{r}) f(\vec{r}) d^{3} \Delta \vec{r}$ gives

$$
q(\vec{r}, s)+\frac{d q(\vec{r}, s)}{d s} \Delta s=q(\vec{r}, s)+\nabla q(\vec{r}, s) \cdot\langle\Delta \vec{r}\rangle+\frac{1}{2} \sum_{i=1}^{3} \sum_{j=1}^{3} \frac{\partial^{2} q(\vec{r}, s)}{\partial r_{i} \partial r_{i}}\left\langle\Delta r_{i} \Delta r_{j}\right\rangle
$$


Simplifying the above by using the fact that, by symmetry of the bonds, we know that $\langle\Delta \vec{r}\rangle=0$. Also, as was found earlier in this work $\left\langle\Delta r_{i} \Delta r_{j}\right\rangle=a^{2} \delta_{i, j} \Delta s$. Substituting these two results into Equation 1.14 simplifies the expression to

$$
\frac{d q(\vec{r}, s)}{d s}=\frac{a^{2}}{6} \nabla^{2} q(\vec{r}, s)
$$

With this, the single-chain partition function $Q=\int_{\mathbb{R}^{3}} q(\vec{r}, N) d^{3} \vec{r}$ can be found from the forward chain propagator $q(\vec{r}, s)$, which gives the probability of segments of the chain being at a position $\vec{r}$. There is also a backward propagator $q^{\dagger}\left(\vec{r}, s^{\prime}\right)$ that starts at the $s=N$ side of the polymer and propagates down the chain towards the other end. The backwards propagator is given by

$$
\frac{d q^{\dagger}(\vec{r}, s)}{d s}=-\frac{a^{2}}{6} \nabla^{2} q^{\dagger}(\vec{r}, s)
$$

Putting together the forward and backward propagators gives the partition function as,

$$
Q=\int_{\mathbb{R}^{3}} q^{\dagger}(\vec{r}, s) q(\vec{r}, s) d^{3} \vec{r}
$$

\subsection{Mean-Field Theory}

The standard Gaussian chain model can be solved by replacing the interactions between the polymers in the melt with interactions of independent polymers with fields. This replaces the instantaneous polymer-polymer interactions with their average interaction. For diblocks, this average interaction is represented by the value of two fields $w_{A}$ representing the average interaction with $A$ type monomers and $w_{B}$ representing the average interaction with $B$ type monomers. This simplifies the model from $n$ interacting polymers in a melt to a system of $n$ non-interacting polymers that are coupled to a field by the energy

$$
U_{\text {field }}=1 / V \int_{\mathbb{R}^{3}} d^{3} \vec{r}\left[\chi N \phi_{A}\left(1-\phi_{A}\right)-w_{A} \phi_{A}-w_{B}\left(1-\phi_{A}\right)\right] .
$$

Adding this field energy to the single-chain partition function, $Q\left[w_{A}, w_{B}\right]$, for a diblock in the fields $w_{A}$ and $w_{B}$ gives the free energy,

$$
\frac{F\left[w_{A}, w_{B}, \phi\right]}{n k_{B} T}=-\ln \left(\frac{Q\left[w_{A}, w_{B}\right]}{V}+\frac{1}{V} \int_{\mathbb{R}^{3}} d^{3} \vec{r}\left[\chi N \phi_{A}\left(1-\phi_{A}\right)-w_{A} \phi_{A}-w_{B}\left(1-\phi_{A}\right)\right]\right),
$$


which can be calculated by evaluating the propagators. The propagators can be found by adding the fields to the propagator Equations 1.15 and 1.16. substituting in the

$$
\begin{gathered}
W(\vec{r}, s)= \begin{cases}w_{A}(\vec{r}, s) & 0 \leq s \leq N f \\
w_{B}(\vec{r}, s) & N f<s \leq N\end{cases} \\
\frac{d q(\vec{r}, s)}{d s}=\frac{a^{2}}{6} \nabla^{2} q(\vec{r}, s)-W(\vec{r}, s) q(\vec{r}, s) \\
\frac{d q^{\dagger}(\vec{r}, s)}{d s}=-\frac{a^{2}}{6} \nabla^{2} q^{\dagger}(\vec{r}, s)+W(\vec{r}, s) q^{\dagger}(\vec{r}, s)
\end{gathered}
$$

However, to solve Equations 1.31 and 1.32, we require the fields $w_{A}$ and $w_{B}$ as well as $\phi_{A}$ and $\phi_{B}$. Finding the field requires solving the following mean-field conditions,

$$
\begin{gathered}
\phi_{A}+\phi_{B}=1, \\
w_{A}-w_{B}=\chi N\left(\phi_{B}-\phi_{A}\right), \\
\phi_{A}=-V \frac{D \ln Q}{D w_{A}}
\end{gathered}
$$

and

$$
\phi_{B}=-V \frac{D \ln Q}{D w_{B}}
$$

The first mean-field condition is local incompressibility. Remembering back to when we first defined a polymer melt, we said that they where incompressible. However, local incompressibility is a stronger condition as it constrains not just the average concentrations to be constant, but forces the instantaneous concentrations everywhere in the melt to be one. The second condition gives the relationship between the mean-fields, $w_{A}$ and $w_{B}$, and the concentrations $\phi_{A}$ and $\phi_{B}$. The final two conditions are the definition for concentration and how to obtain them from the single-chain partition function $Q$.

The mean-field theory is very powerful. Possibly one of its biggest triumphs is random phase approximations calculations of the disordered state structure functions. The random phase approximation was applied to diblocks by Ludwik Leibler in 1979 [12], He used a fourth-order weak inhomogeneity expansion of the free energy of diblocks to calculate the stable phases, as well as the structure functions. The structure function, also called the 
scattering function, is observed in small angle scattering experiments for the disordered phase. The structure function is the spherically averaged reciprocal space representation of the configuration of the melt. The random phase approximation structure function is

$$
S(k)=\frac{W(k)}{s(k)-2 \chi W(k)} .
$$

Where $W(k)=s_{A, A}(k) s_{B, B}(k)-s_{A, B}(k) s_{B, A}(k)$ and $s(k)=s_{A, A}(k)+s_{B, B}(k)+s_{A, B}(k)+$ $s_{B, A}(k)$. These can both be derived from the tensor $s_{i, j}(k)$ defined as

$$
\begin{gathered}
s_{A, A}(k)=N g_{1}(x, f), \\
s_{B, B}(k)=N g_{1}(x, 1-f),
\end{gathered}
$$

and

$$
s_{A, B}(k)=s_{B, A}(k, 1-f)=\frac{N}{2}\left[g_{1}(x, 1)-g_{1}(x, f)-g_{1}(x, 1-f)\right] .
$$

With $x=k^{2} a^{2} N / 6$ and $g_{1}(f, x)$, the Debye function is defined as $g_{1}(x)=2\left[f x+e^{-f x}-\right.$ 1] $/ x^{2}$. Later, the process was generalized for polydisperse melts by Hong and Noolandi [7]. The result of this change is to replace $g_{1}(f, x)$ by the number-average Debye function

$$
\bar{g}_{1}(\bar{f}, \bar{x})=2\left[\bar{f} \bar{x}+(1+\bar{f} \bar{x} K)^{-K^{-1}}-1\right] / \bar{x}^{2}
$$

Equation 1.37 can then be simplified by substituting $F(k)=N s(k) / W(k)$ to give

$$
S(k)=\frac{N}{F(k)-2 \chi N} .
$$

The over bars represent number-averages of quantities, so $\bar{x}=k^{2} a^{2}<N>_{n} / 6$ and $\bar{f}=$ $<f>_{n}$. The polydispersity index $Đ$ and $K$ are related by $K=(Đ-1)^{-1}$. With this we have a polydisperse scattering function for diblock copolymers.

Another important result from mean-field theory is the calculation of a phase diagram for diblocks in Figure 1.5. The phases diagram has a number of interesting features. There is a critical point at $f=0.5$ and $\chi N=10.495$ where all the phase boundaries coexist and there is a direct transition between the disordered phase and ordered lamellar phase. However, over the rest of the range in $f$, there are only order-disorder transitions (ODTs) to the sphere phase. This is important because it contradicts the experimental evidence where direct transitions are observed to the cylinder and complex phases. The reason for 
this contradiction is that the mean-field theory approximates the $w_{A}$ and $w_{B}$ fields with their averages, neglecting fluctuations in the fields.

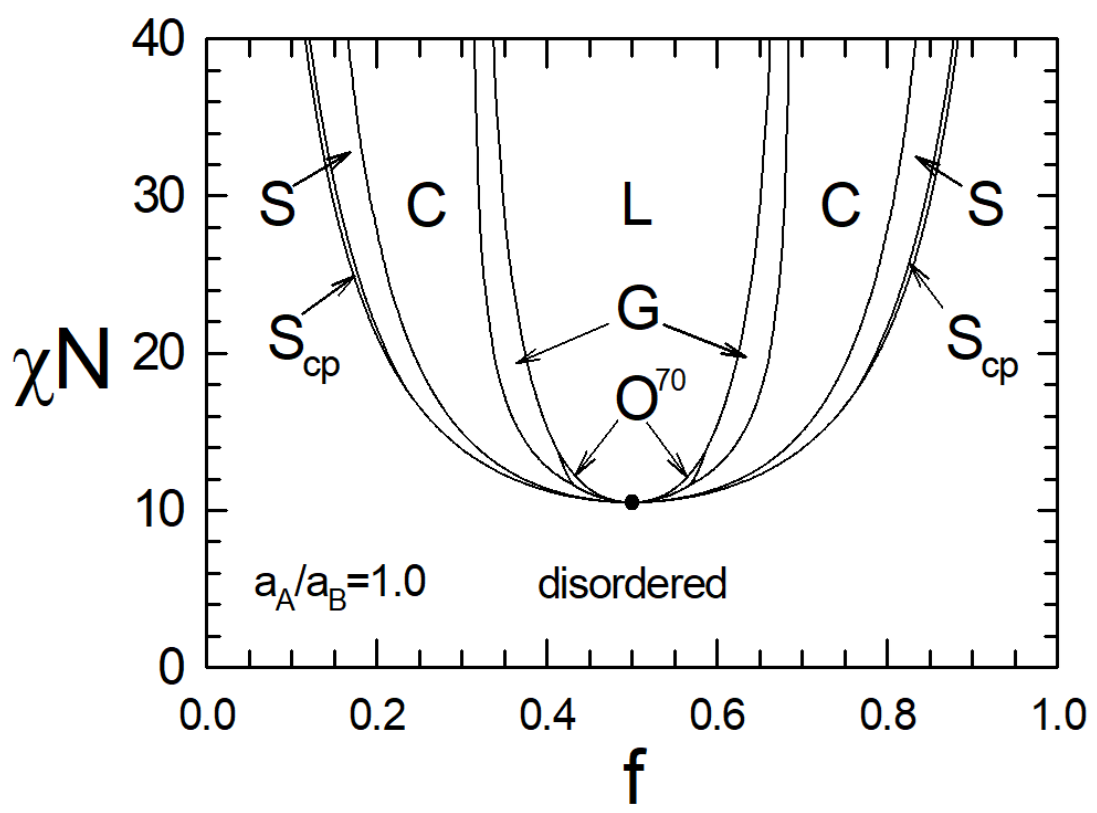

Figure 1.5: Diblock copolymer phase diagram reproduced from Matsen and Bates [13].

\subsection{Fluctuations}

The mean-field theory for diblock copolymers is a powerful tool. However, it makes some assumptions that become inaccurate as the chains become shorter. Melts with shorter chains have larger fluctuations in their fields [15], and so it becomes inaccurate to approximate the polymer melt with its most likely configuration of there fields, as is done in mean-field theory. The size of this deviation from mean-field theory are controlled by the invariant polymerization index, $\bar{N}=\left(\rho_{0} a^{3}\right)^{2} N$. To correct for this, a sum over all possible field configurations according to their Boltzmann weights, would have to be done. This is the approach taken by some computer simulation methods [3, 17]. However, there do exist analytic theories capable of approximating the fluctuating fields using perturbation theory.

The first fluctuation correction to the phase diagram for diblocks was by Fredrickson and 
Helfand [15]. Their theory used the same fourth-order expansion as the Leibler randomphase approximation for the single-chain partition function, but also include Gaussianorder fluctuations by use of the Hartree approximation [16], allowing the model to make better predictions for the disordered phase. It predicted the order-disorder transition for symmetric diblocks as

$$
(\chi N)_{O D T}=10.495+41.0 \bar{N}^{-1 / 3} .
$$

This theory also allowed for the prediction of a phase diagram for fluctuating diblock copolymers. This phase diagram has a number of important features. The first is that there is no longer a critical point at $f=0.5$. There are also direct transitions between the disordered phase and the non-spherical ordered phases. This is more in line with experimental evidence, which finds the direct transitions between the disordered and nonspherical ordered phases.

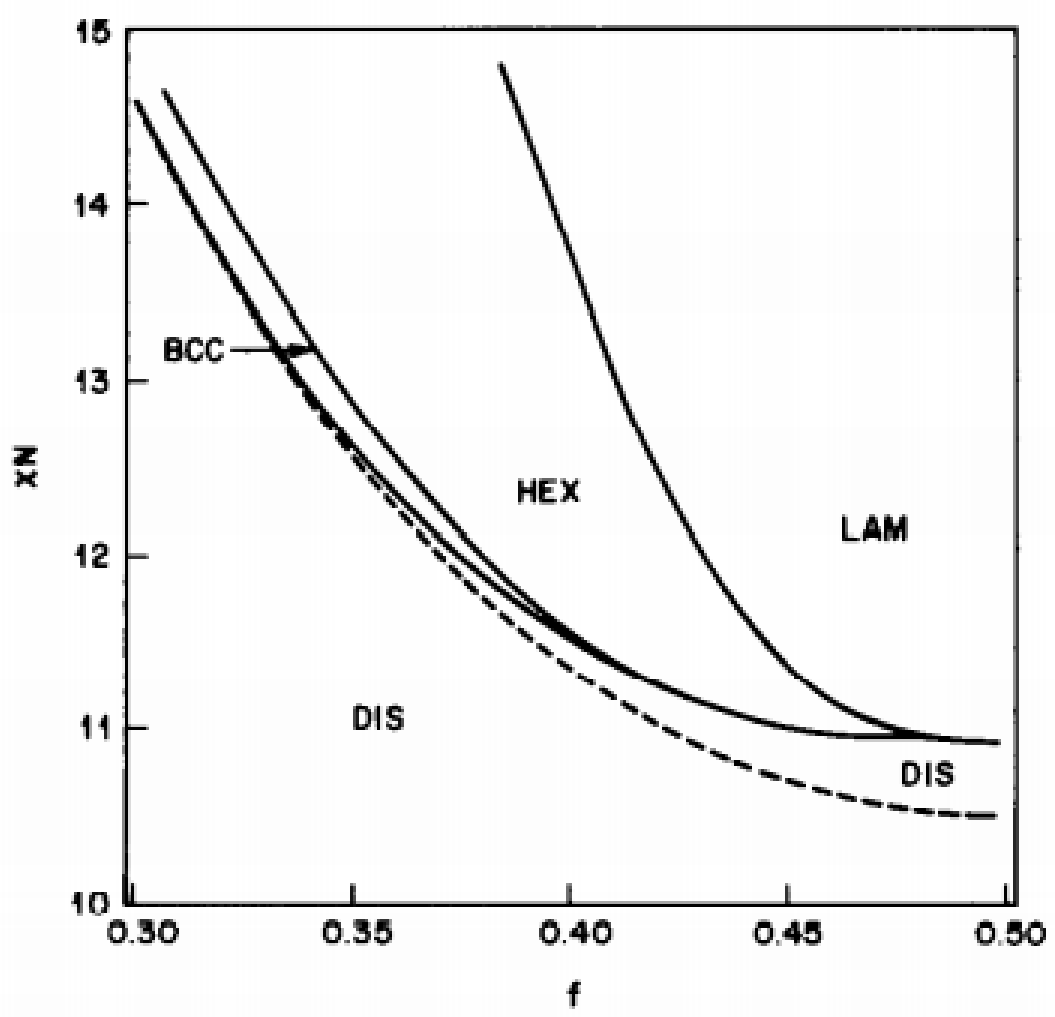

Figure 1.6: Diblock copolymer phase diagram reproduced from Fredrickson-Helfand 1989 [15]. 
The newer renormalized one-loop theory(ROL) has improved on the Fredrickson-Helfand theory $[18,19,20]$. This new theory has provided more accurate predictions for the disordered-state structure function of diblock copolymers. The renormalized one-loop theory is also based on a perturbation approach. As well as the renormalization of the parameters $\chi$ and $a$, where $\chi$ is the interaction parameter and $a$ is the statistical segment length. This improved method allows for more accurate structure function calculations in the disordered state.

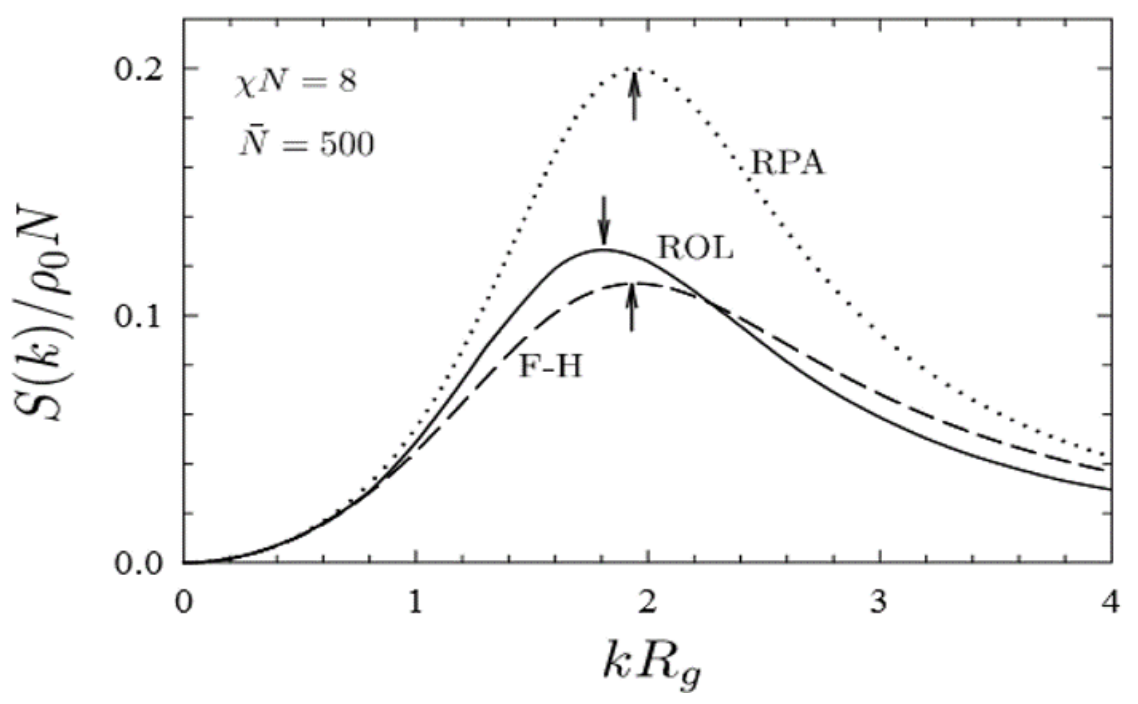

Figure 1.7: Comparison of the disorder state structure functions for the three different theories $[12,15,21]$.

The above comparison of the structure functions for different methods show how they differ at low $\bar{N}$. However, there has not been a calculation of the phase diagram from the renormalized one-loop theory. The phase diagram can not be calculated because of the difficulty of treating the ordered diblock phases with the one-loop method. However, the one-loop theory gives very accurate predictions for the structure function that can be used to calibrate other systems, essentially renomalizing their $\chi$ values [18].

\subsection{Universality}

It has been shown that all models of diblock copolymer melts behave identically to the standard Gaussian chain model in the limit of large $N$ [18, 22, 23, 24]. This fact is called 
universality. It is an extremely useful result because it means there is a mapping between the model specific parameters and a set of universal parameters $\left(\chi N, f, \bar{N}, \gamma_{A B}\right)$ of the standard Gaussian chain model. The fact that all systems, theoretical and experimental, can be mapped onto the same set of universal parameters allows for quantitative predictions for any experimental system using any theoretical model. The only caveat is that the degree of polymerization needs to be sufficiently large for universality to hold.

The universality of polymer melts was used to find a more accurate value for the orderdisorder transition for symmetric diblocks, by calibrating a number of computer simulations to the ROL theory [22]. This calibration involved fitting the statistical segment length to predictions of the renormalized one-loop theory, as well as fitting the structure functions to ROL to find the interaction parameter $\chi$. Subsequently, the calibrated simulations were used to find the order-disorder transition in terms of the calibrated parameters. The simulated order-disorder transitions were used to find a correction to the FredricksonHelfand prediction of the form $C_{1} \bar{N}^{-C_{2}}$, as shown in Figure 1.8 by the dotted line.

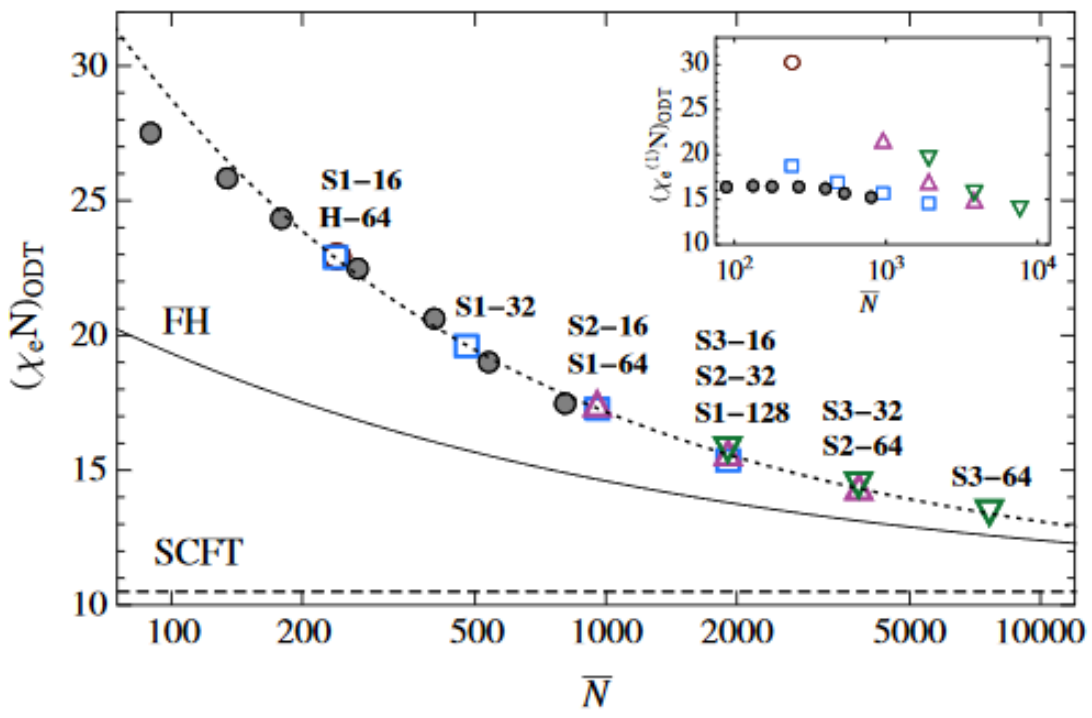

Figure 1.8: Plot of the ODT of symmetric diblocks over a range of $\bar{N}$. [18]. Different symbols are from different models that where calibrated independently.

The correction shown in Figure 1.8 is of the form,

$$
(\chi N)_{O D T}=10.495+41.0 \bar{N}^{-1 / 3}+123 \bar{N}^{-0.56} .
$$


This gives a more accurate prediction of the order-disorder transition of monodisperse diblock copolymer melts. Once these models are calibrated, they can be used to make predictions for any polymer system. This prediction can be applied not only to simulations, but also to polymer systems studied in experiments. However, the calibrations of experiments requires a determination of $\chi$, which has been a longstanding problem.

the universality lets experimental systems be mapped onto the standard Gaussian chain model. This mapping requires a calibration of the Flory-Huggins parameter $\chi$. There are numerous strategies used to calibrate the $\chi$ parameter, but this has lead to a discrepancy between the values found using different methods [25, 26, 27, 28, 29, 30]. This has caused many to propose that $\chi$ depends not only on temperature, $T$, but also on architecture, composition, molecular weight, and polydispersity. This is problematic, because if it were true, analytical theories and simulations would lose the ability to make quantitative predictions, since every comparison with theory would require a separate calibration. This issue comes from the way $\chi$ is determined in experiments.

The determination of $\chi$ involves measuring some quantity by experiment and then fitting to a prediction of the standard Gaussian chain model. Therefore, an accurate $\chi$ requires not only an accurate experimental measurement of the quantity, but also an accurate prediction from the standard Gaussian chain model. As a result of using a prediction from mean-field theory, the calibration hides the fluctuation corrections, which are ignored in mean-field theory, in the value of $\chi$. This would, in effect, give $\chi$ a dependence on molecular weight, architecture, composition, and polydispersity since the fluctuation correction depend on all these parameters.

This work will correct this common inaccuracy in the calibration, by calibrating against a prediction for the order-disorder transition that accounts for fluctuation, polydispersity, and compositional asymmetry. To find this prediction, we will correct the universal curve, Equation 1.44, for polydispersity and compositional asymmetry. The correction for polydispersity is found using a lattice model to run simulations over the relevant range of polydispersity indices. A similar correction for compositional asymmetry is found using data from the literature [31]. 


\section{Chapter 2}

\section{Monte Carlo Simulations}

In the Introduction, it was shown that a polymer melt of density $\rho_{0}$ consists of $\mathrm{n}$ polymers. A number of techniques exist to simulate polymer melts, however, probably the most direct are particle-based simulations. Particle-based methods can model the polymer chains in a variety of ways, one of which involves constraining the segments of the polymer to a lattice. This has the advantage of speeding up the simulation by reducing the possible states of the system, as well as the intrinsic speed advantage from integer computing.

\section{$2.1 \quad$ Lattice Model}

Throughout this work, we perform our simulations on a lattice, where the location of the monomers can be tracked with a single integer number, $l$, the lattice site number. The simulation lattice has a face-centered-cubic (FCC) symmetry, created by removing every other site in a simple cubic lattice. This means that each monomer has coordinates $\vec{r}_{l}=(i, j, k)$, where $i+j+k$ is even. This FCC lattice has a bond length, $b$, between lattice sites that is $\sqrt{2}$ times what it would be on the simple cubic lattice. An FCC lattice is used because it gives the polymers a large number of nearest-neighbour sites, allowing more possible bond angles and a more flexible polymer. As a result, the molecules can approximate the Gaussian chain model with fewer chain segments. This simulation is carried out in a cubic simulation box with a finite side length, $L$, meaning that $i, j$ and $k$ are integers in the range $[0, L]$. To approximate the bulk behavior of an infinite lattice, periodic boundary conditions are used between opposite sides of the cube. The volume of the cubic box is the product of the side lengths 


$$
V=(L)^{3}=\frac{b^{3} M}{\sqrt{2}},
$$

where $M$ is the number of allowed sites on the lattice. The polymers in the system consist of monomers connected between nearest-neighbour sites. There is a set monomer density of $\rho_{0}$, defined as the number of monomers divided by the volume of the lattice, $\rho_{0}=\frac{n_{\text {mono }}}{V}$. By allowing $c$ monomers per lattice site higher densities can be reached, since this gives the box a total number of sub-sites of $c M$. To allow room for the polymers to move, $20 \%$ of the sites are left with one vacancy. This gives a total number of monomers of $\left(c-\frac{1}{5}\right) M$, and so a density of

$$
\rho_{0}=\frac{n_{\text {mono }}}{V}=\frac{\left(c-\frac{1}{5}\right) \sqrt{2}}{b^{3}} .
$$

The polymers on the lattice are diblocks, meaning that there is a contribution to the energy from contacts between the $A$ and $B$ type monomers in the systems that can be expressed as

$$
\frac{E}{k_{B} T}=\frac{\epsilon}{k_{B} T} n_{A B}=\alpha n_{A B},
$$

where $\epsilon$ is the energy per contact and $\alpha$ is the dimensionless energy per site, defined as $\frac{\epsilon}{k_{B} T}=\alpha$. To find this energy, the number of $A-B$ contacts must be calculated by summing over all contacts,

$$
n_{A B}=\sum_{i=1}^{V} \sum_{j=1}^{V} \Delta_{i, j} n_{A, i} n_{B, j},
$$

where $n_{A, i}$ is the number of $A$ monomers in site $i$ and $n_{B, j}$ is the number of $B$ monomers in site $j$. The range of the interactions is controlled by the tensor $\Delta_{i, j}$. To best match the standard Gaussian chain model, the range of the interactions needs to be short relative to the length of the polymers. When $c$ is larger than two, we make the interactions pointlike so only monomers on the same lattice site interact. This point-like interaction makes the tensor equal to $\Delta_{i, j}=\delta_{i, j}$, the Kronecker delta function. However, this is not possible when the number of monomers on a lattice site is small. In this case, the nearest-neighbour interactions between monomers are also needed, giving the tensor $\Delta_{i, j}=1$ if $\left|\overrightarrow{r_{i}}-\overrightarrow{r_{j}}\right| \leq b$. This work uses four different values of the number of monomers per site $c$. If $c=3,5$ then we use only the same site interaction. However, if $c=1,2$ then we included the nearest-neighbour interactions. 
To model polydisperse melts, the simulated polymers in the lattice model will need to have different chain lengths. The distribution of polymer chain weights can be set by starting the simulation with the correct distribution in its initial configuration. One complication is that the Schultz-Zimm distribution used in this work is continuous, whereas the lattice model has polymers made of discrete sections. To approximate the desired distribution, the continuous distribution can be divided into bins with length 1 . This gives the discretization of the distribution for $i=A$ and $B$ as

$$
n_{N_{i}}=\frac{K^{K}}{\left(N_{n}\right)^{K} \Gamma(K)} \int_{N_{i}-0.5}^{N_{i}+0.5} N^{K-1} e^{-\frac{K N}{\left(N_{n}\right)}} d N .
$$

Note that for $N_{i}=0$ the bin is defined by half the width

$$
n_{0}=\frac{K^{K}}{\left(N_{n}\right)^{K} \Gamma(K)} \int_{0}^{0.5} N^{K-1} e^{-\frac{K N}{\left(N_{n}\right)}} d N
$$

Where $\Gamma(K)$ is the gamma function and $K=1 /(Đ-1)$ is the relationship between $K$ and polydispersity index $\mathrm{Ð}=N_{w} / N_{n}$. A correction is then added so the total number of chains, $n$, and average chain length, $N_{n}$, are correct. Then to optimize the distribution further, we minimize

$$
\Xi\left(n_{N_{i}}\right)=\sum_{N_{i}}\left[n_{N_{i}}-n p_{i}\left(N_{i}\right)\right]^{2}+\lambda\left[n N_{n}^{2} \bigoplus_{i}-\sum_{N_{i}} n_{N_{i}} N_{i}^{2}\right]^{2},
$$

where the first term matches the discretized Schultz-Zimm distribution, $n_{N_{i}}$, to its continuous analog $p_{i}(N)$ and the second term matches the polydispersity index to the desired value of $\mathrm{Ð}_{i}$. The coefficient $\lambda$ which dictates the relative importance of the two contributions, is set to $10^{-7}$ [32].

To generate the distributions for diblocks, this method for finding the distribution is

used separately on both the $A$ and $B$ type monomers to form two distributions $n_{N_{A}}$ and $n_{N_{B}}$. Polymers are then sampled from these distributions at random and joined together to form diblocks, where each block separately has the discretized Schultz-Zimm distribution and the blocks are completely uncorrelated.

\subsection{Statistical Mechanics}

Statistical mechanics show that systems in equilibrium are described by Boltzmann distributions. The lattice model has a large number of discrete states corresponding to all 
possible configurations of polymers on the lattice. Each state is labeled $i$ and has energy $E_{i}$ proportional to the total number of A-B contacts. The simulations are performed at a temperature $T$ and $k_{B}$ is Boltzmann's constant. The probability of a system in equilibrium being in the $i^{\text {th }}$ state is given by the Boltzmann weight

$$
p_{i}=\frac{1}{Z} e^{\frac{-E_{i}}{k_{B} T}}
$$

where $Z$ is the partition function, a central quantity in statistical mechanics defined as

$$
Z=\sum_{i} e^{\frac{-E_{i}}{k_{B} T}}
$$

The lattice model has an energy $E_{i}=\epsilon n_{A B}$ that is equal to the number of contacts between $A$ and $B$ monomers, $n_{A B}$, times an energy per contact $\epsilon$. This can be simplified by introducing the dimensionless energy per contact. By rewriting Equation 2.8, the probability of a state $i$ is

$$
p_{i}=\frac{1}{Z} e^{\frac{-\epsilon}{k_{B} T} n_{A B}}=\frac{1}{Z} e^{\alpha n_{A B}}
$$

To find a macroscopic observable, we need the expectation value of some quantity $O_{i}$,

$$
\langle O\rangle=\frac{1}{Z} \sum_{i} O_{i} e^{-\alpha n_{A B}},
$$

where the sum is over all possible configuration of polymers on the lattice. However, the configuration space of the lattice is high dimensional and so the sum can not be calculated by direct methods. To give a good approximation to this sum, this study uses Monte Carlo methods, which work by sampling a representative subset of the sums terms. In our case, this is equivalent to sampling a subset of all possible configurations of polymers on the lattice.

\subsection{Monte Carlo Steps}

The simulations in this work use the Metropolis algorithm to find the average of any desired observable, $\langle O\rangle$. This algorithm approximates Equation 2.11 by randomly sampling $O_{i}$ according to the Boltzmann distribution [33, 34]. It does this by constructing a Markov chain

that randomly moves through the possible configurations of the polymers on the lattice by applying randomly selected Monte Carlo moves. To achieve this on the polymer lattice 
model, we need to pick a set of Monte Carlo moves that satisfy two important criteria. The first criteria is ergodicity, the property that any possible configuration of polymers on the lattice could be reached from any other configuration by repeatedly applying the Monte Carlo moves. The second property is detailed balance, which is satisfied if the probability of the system to transition into a state $i$ from a state $j$ is equal to the probability of it transitioning back to $j$ from $i$.

The Metropolis algorithm achieves detailed balance by specifying the transition probability $\Pi_{j \leftarrow i}=A(j, i) h(j, i)$. Here $\Pi_{j \leftarrow i}$ is the total probability of the move being performed. $h(j, i)$ is the probability of trying a move at the start of the Monte Carlo step and $A(j, i)$, the acceptance criterion, is the probability that a given Monte Carlo move is performed after we try it. The acceptance criterion compares the ratio of the Boltzmann weights to a uniform random number in the range $[0,1]$

$$
R_{[0,1]}<\min \left(e^{\frac{-\epsilon\left(n_{A B, j}-n_{A B, i}\right)}{k_{B} T}}, 1\right),
$$

and is defined such that the probability of transitioning in and out of any state is the same. Therefore, the simulation will respect detailed balance as long as the forward and backwards moves are attempted with equal probability.

To start each Monte Carlo step, a random polymer is picked out of the melt. A move is randomly picked out of a list of four moves. We us a standard set of Monte Carlo moves [33, 32, 35], they are the slithering snake move attempted $40 \%$ of the time, the crankshaft move attempted $40 \%$ of the time, the flip move attempted $10 \%$ of the time, and the polymer rebridging move also attempted $10 \%$ of the time.

In the slithering snake move, either the head or tail of the polymer is picked at random. Then one of the twelve nearest-neighbour sites are picked and the move is attempted. If there is not a vacancy the move is rejected, unless the site contains the other end of the polymer being moved. In the latter case, the move is accepted because the other end monomer will move out of the site to make room. If the move is accepted, the end of the polymer is moved into it and the rest of the monomers are shifted one place along the chain contour towards the end that has moved. This move satisfies detailed balance because the probability of moving the head into a new location is the same as the probability of undoing this move by moving the tail back to the vacancy it left at its old location. This move alone makes the set of Monte Carlo moves ergodic because by its repeated application we can move any polymer from any location and configuration to any other location and configuration. when applied to all polymers in the melt, any configuration can then be reached. 

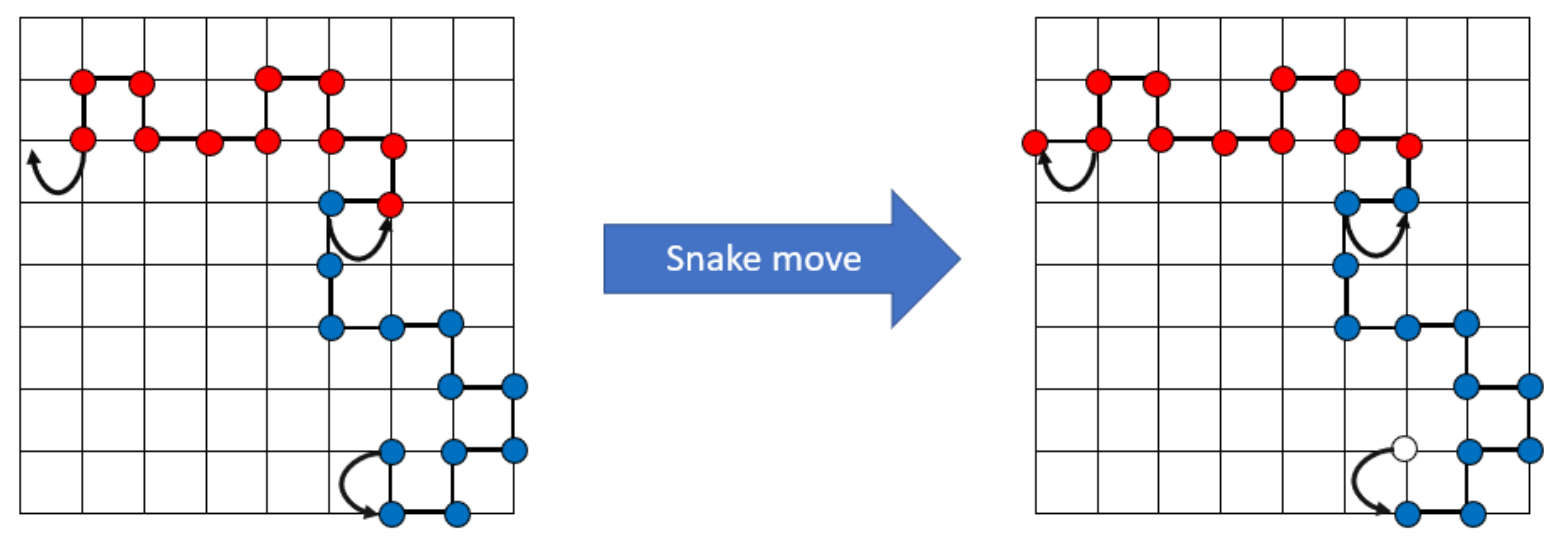

Figure 2.1: Example of the slithering snake move. For simplicity, the move is demonstrated on a two-dimensional simple square lattice

The second Monte Carlo move is the crankshaft. In this case, one of the non-end monomers along the chain is picked at random. Then all of the sites which are common nearest-neighbors to its two adjacent monomers on the chain are tested for vacancies. This checks all possible bonds this monomer could make in the chain. There are five different bond angles on an FCC lattice, $180^{\circ}, 120^{\circ}, 90^{\circ}, 60^{\circ}$ and $0^{\circ}$ degrees, having $1,2,4,4$ and 12 bonds with these five different angles respectively. So, if the randomly chosen monomer is at a $90^{\circ}$ or $60^{\circ}$ angle, then we have to check $4-1=3$ possible sites for vacancies. However, if the chosen monomer is at a $180^{\circ}$ angle, then there is no possible move and the crankshaft is rejected. If it is the case that there are vacant sites available, one of them is picked at random and the move attempts to put the selected monomer in that site. The crankshaft preserves detailed balance because the probability of attempting the reverse move is the same as the original move, as the number of possible bonds has not changed. 

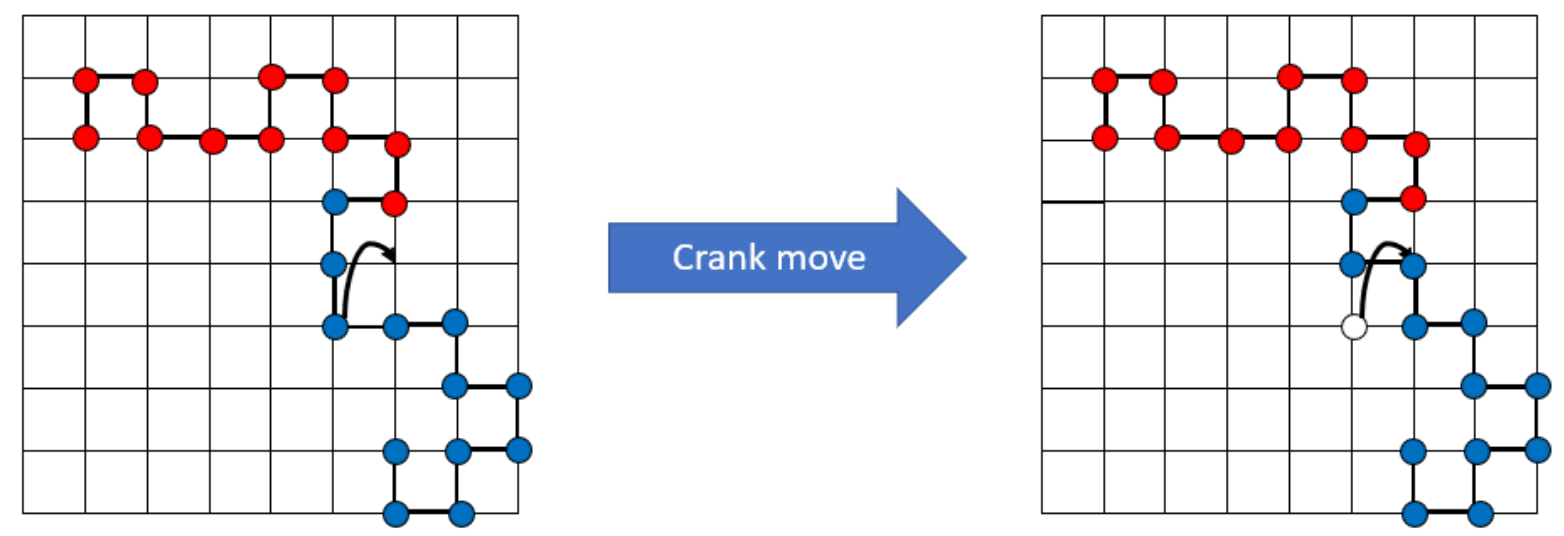

Figure 2.2: Example of the crankshaft move. For simplicity, the move is demonstrated on a two-dimensional simple square lattice

The third Monte Carlo move is the flip move. The polymer is flipped head over tail so that a monomer a distance $i$ from the head is swapped with the monomer a distance $N-i$ from the head. This move obeys detailed balance because the probability of performing a flip on the polymer both before and after the move is simply the constant probability of picking that polymer from the melt. The head-to-tail flip makes a larger change to the system than the other moves, but also requires more computational time. Also, due to the potential large changes in energy it can cause, it has a lower acceptance rate than the other moves in the ordered phase. 

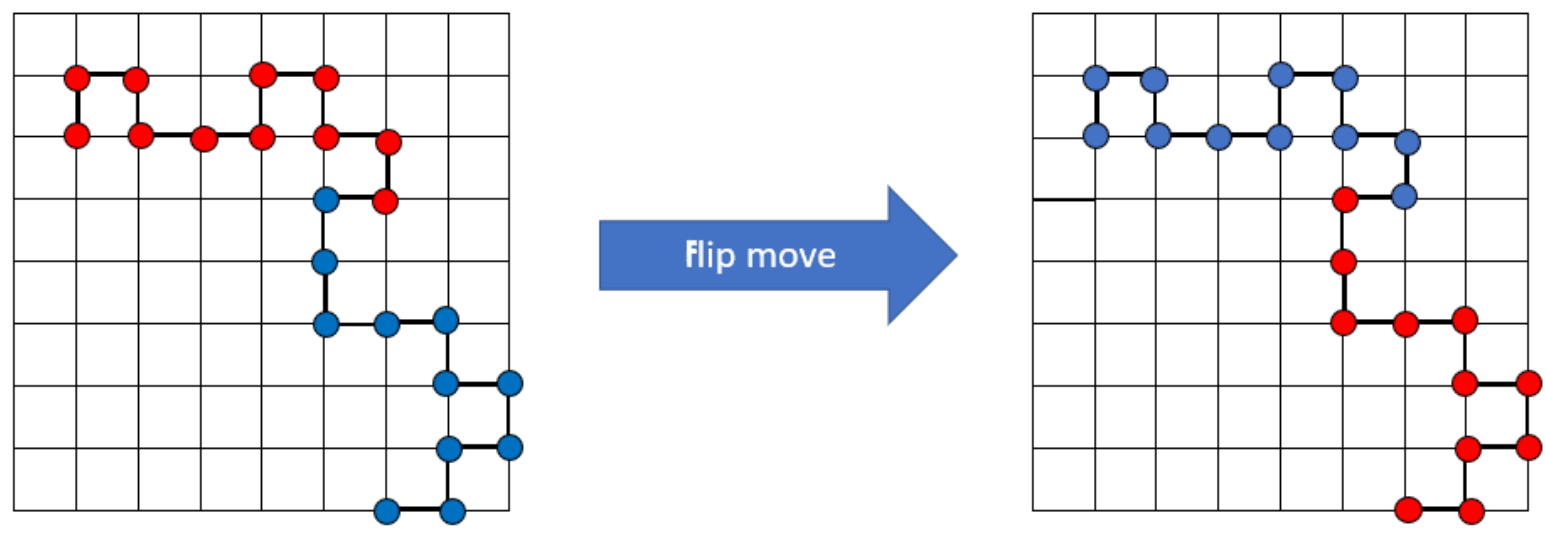

Figure 2.3: Example of the head-to-tail flip move. For simplicity, the move is demonstrated on a two-dimensional simple square lattice

The last Monte Carlo move is the polymer rebridging move. A site is randomly selected, and for each monomer on that site we calculate the distance from the head/tail of its parent molecule. If the positions of two monomers along their respective chains are equal, then the chain ends are exchanged between the two polymers so that the monomers closer to the end being swapped are traded between chains. This move can not be performed on simulations where the number of monomers per site is one. In this case, the probability of attempting this move is split equally between the crankshaft and slithering snake move. The rebridging move respects detailed balance because performing the exchange does not change the number of polymer-polymer interactions, so the probability of undoing the move after it is performed is the same as preforming the move. 


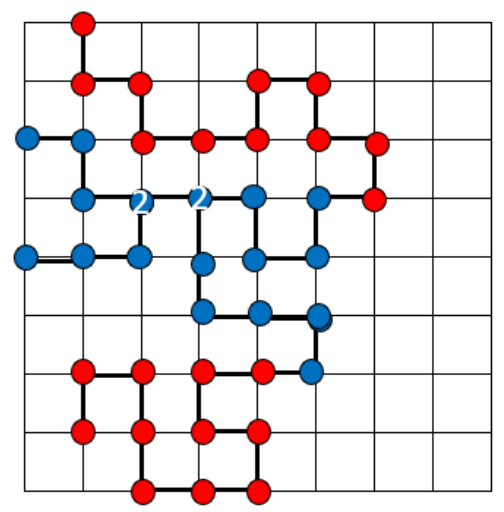

\section{Rebridging move}

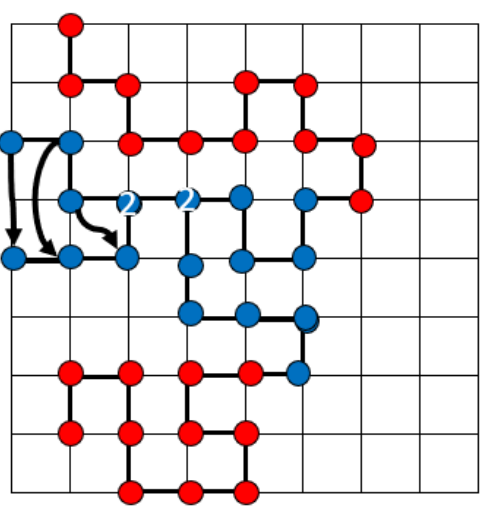

Figure 2.4: Example of the polymer rebridging move. For simplicity, the move is demonstrated on a two-dimensional simple square lattice. The sites with two monomers on them are marked with the number 2 .

The Monte Carlo Metropolis algorithm follows these general steps:

- The melt is in some configuration $j$ with energy, $E_{j}$.

- Pick one of the Monte Carlo moves by their associated probability and perform it on the melt to get a new energy $E_{i}$.

- Calculate the difference in energy $\Delta E=E_{i}-E_{j}$, and if the energy has decreased or stayed constant the move is accepted. If the energy has increased, generate a random number, and if it is less then $e^{-\Delta E / k_{B} T}$ the move is accepted.

- Record relevant data to find averages.

- Repeat previous steps.

\section{$2.4 \quad$ Parallel Tempering}

One difficulty the lattice model simulations of ordered phases have is that the system can form long-lived defects. Such defects have large energy barriers that the simulation needs to overcome to reach the equilibrium state. One way to address this issue is to perform 
an ensemble of simulations with different values of the interaction parameter, $\alpha$, known as a parallel tempering simulation. These simulations consists of a set of $W$ replicas of the system, each spaced in $\alpha$ by the value $\delta \alpha$. Because the replicas are independent, the total partition function of the joint system is the product of the independent partition functions

$$
Z_{\text {total }}=\prod_{j=1}^{W} Z_{j} .
$$

In order to reach equilibrium faster, we add a Monte Carlo move to swap configurations with neighbouring values of $\alpha$. The swap acceptance probability is the same as the other Monte Carlo moves, where the probability of swapping is proportional to the ratio of the Boltzmann weights.

$$
P_{i, j}=\frac{e^{\alpha n_{A B, j}}}{e^{(\alpha+\delta \alpha) n_{A B, i}}} .
$$

Detailed balance is maintained by the fact that the replicas to exchange are always selected randomly with a probability of $1 /$ (\# replicas -1$)$. Therefore swapping back is just as frequency to be attempted as the original swap is. These swaps are attempted every 1000 Monte Carlo step per monomer.

The implication of this move is that if one of the configurations at a higher interaction parameter forms a defect, that configuration can swap to a lower value of interaction parameter because it will have a higher number of contacts than an ordered phase at a lower value of the interaction parameter. After the swap, the lower value of interaction parameter will lower the energy barrier, so that the speed of removing the defect becomes faster. This means that, with parallel tempering, the lattice model simulations will have shorter lived defects and the simulation will come to equilibrium faster. This is the main advantage of running parallel tempering simulations.

Another notable benefit is the fact that parallel tempering allows us to leverage the ability of parallel computation. Each of the replicas can be run on a separate logical thread and communication only needs to happen when the replica swap is being attempted, resulting a minimal hit to performance and allowing near linear reduction in time with the amount of CPU resources used. This means that a wide range of the interaction parameter can be investigated simultaneously.

The next question to ask is how to take thermodynamic averages in a parallel tempering simulation. We know that each replica independently has five Monte Carlo moves acting on it: the snake move, crankshaft move, flip move, polymer rebridging move, and the one side of the replica swap. This means each replica is undergoing an independent Monte 
Carlo simulation, so we can take averages over each replica independently to get the value of the desired thermodynamic quantity.

We have created a simulation model well-equipped to simulate polymer melts in terms of the model-specific parameters; the chain length, $N$, and the interaction parameter $\alpha$. The addition of parallel tempering also facilitates the investigation of wide ranges of the interaction parameter to determine phase behaviors, and thus locate the order-disorder transition. However, the predictions of the lattice model will necessarily be in terms of the quantities $N$ and $\alpha$. To make predictions for the universal behavior, we now need the relationship between these parameters and the universal set of parameters. 


\section{Chapter 3}

\section{Calibration of the Lattice Model}

To make general predictions for polymer melts with the lattice model, it is best to express our results in terms of a set of universal parameters. The Morse calibration to the standard Gaussian chain model makes this possible [18]. It requires finding two parameters. The first is the statistical segment length $a$, which is needed to evaluate the value of $\bar{N}=a^{6} \rho_{0}^{2} N$ that controls the magnitude of fluctuations in the melt. The other parameter that must be calibrated is $\chi$, the Flory-Huggins parameter that characterizes the interaction between unlike polymer segments.

\subsection{Calibration for Statistical Segment Length}

To find the segment length of polymers in the lattice model, a prediction for the standard Gaussian chain model in terms of $a$ must be fit to results from the lattice simulation. The Morse calibration uses the relation between $N$ and $R_{g}$, the radius of gyration [22]. Earlier in this work it was shown that for a polymer in a melt, $R_{g}^{2}=\frac{a^{2}}{6} N$. However, a more accurate result from the ROL theory gives a correction to this for finite $\bar{N}$ [36]:

$$
\frac{6 R_{g}^{2}}{N}=a^{2}\left(1-1.42 \bar{N}^{-0.5}+O\left(\bar{N}^{-1}\right)\right) .
$$

With this we can calibrate the statistical segment length by running a set of lattice simulations of polymers with different chain lengths of $N=16,20,30,40,60,100$, and 200 and with the interaction parameter set to $\alpha=0$. This corresponds to the athermal case where 
there are no $A-B$ interactions, and thus the simulation is actually of a homopolymer melt. The procedure involves collecting data for the radius of gyration and fitting it to

$$
\frac{6 R_{g}^{2}}{N}=a^{2}\left(1-1.42\left(a^{6} \rho_{0}^{2} N\right)^{-0.5}+\gamma N^{-1}\right),
$$

where $\gamma$ and $a$ are fitting parameters. Doing this for four different values of $c=1,2,3$, and 5, we obtain the results in Figure 3.1 below.
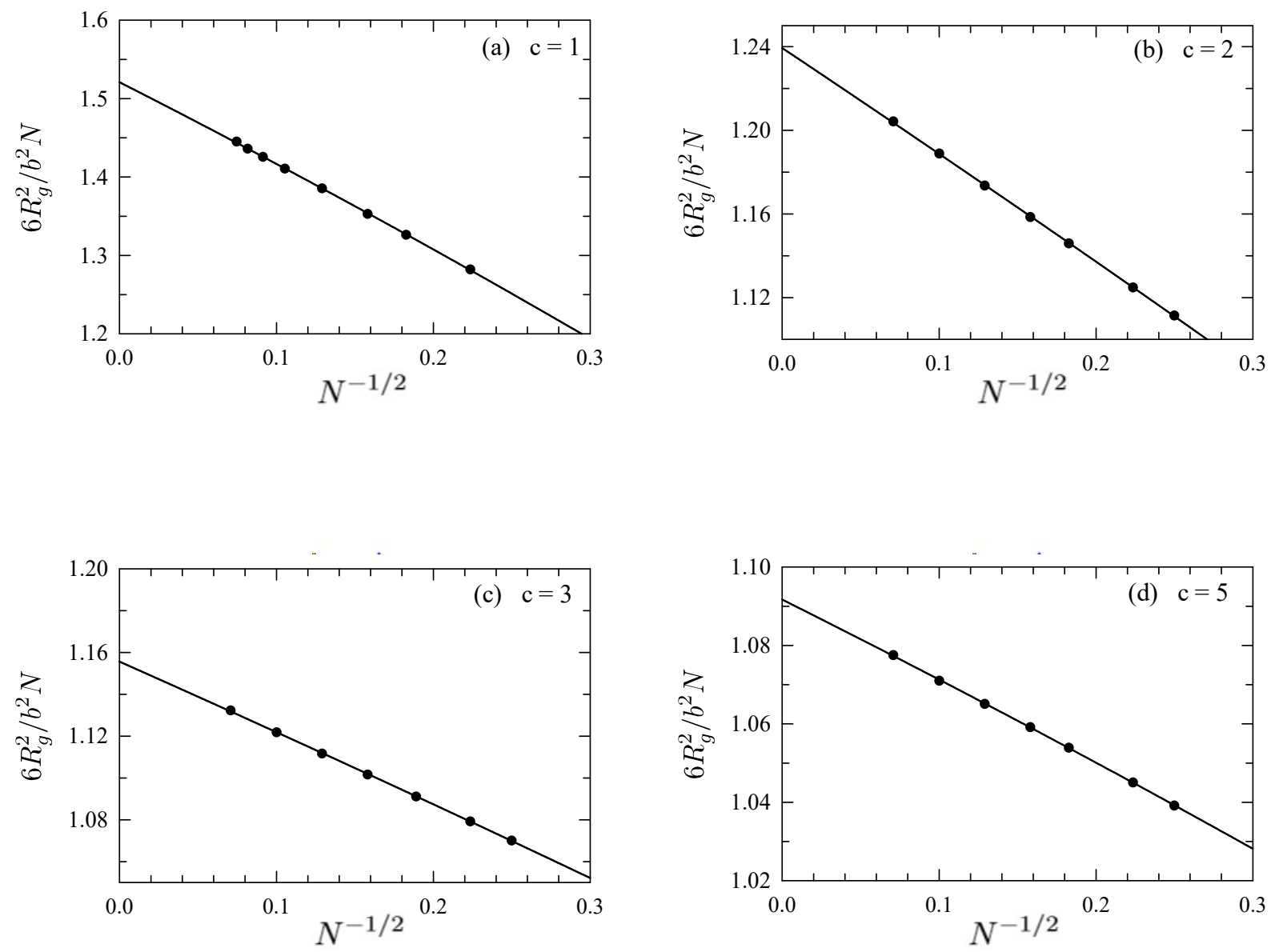

Figure 3.1: Calibration of the statistical segment length for $\mathrm{c}=1,2,3$, and 5 . The symbols are from the lattice simulations and the lines are the fits. 
By plotting $R_{g}^{2}$ as a function of $N^{-1 / 2}$, we obtain the square of the statistical segment length, $a^{2} / b^{2}$, from the $y$-intercept intersect of the Figure 3.1. At the $y$-intercept, the value of $N^{-1 / 2}$ is zero so it corresponds to the radius of gyration at infinite chain length. We can see in Table 3.1 that the statistical segment length decreases with increasing $c$. This is because allowing more monomers per site allows the polymer to cross itself more easily thus acts more flexible and so it adopts a more compact configuration with a smaller $R_{g}$.

Calculation of $\bar{N}=a^{6} \rho_{0}^{2} N$ is required to gauge the size of the fluctuation corrections. Because the simulations will be run at different chain lengths $N$, it is most convenient to evaluate the ratio $\bar{N} / N=a^{6} \rho_{0}^{2}$ for each value of $c$, as shown in Table 3.1. This then allows for simple calculations of $\bar{N}$ for the rest of this work.

\begin{tabular}{||cccc||}
\hline$c$ & $a / b$ & $\rho_{0} b^{3} / \sqrt{2}$ & $\bar{N} / N$ \\
\hline \hline 1 & 1.233 & 0.8 & 4.51 \\
\hline 2 & 1.113 & 1.8 & 12.4 \\
\hline 3 & 1.075 & 2.8 & 24.2 \\
\hline 5 & 1.045 & 4.8 & 59.9 \\
\hline
\end{tabular}

Table 3.1: $c$ is maximum the number of monomers per lattice site, $a / b$ is the statistical segment length relative to the bond length. $\rho_{0}$ is the monomer density, and $\bar{N} / N$ is the ratio of invariant polymerization index to contour length $N$.

\subsection{Calibration of $\chi$}

This section focuses on the calibration of the interaction parameter and is broken into two parts. The first part finds a linear approximation $\chi=z_{\infty} \alpha$ that gives an accurate prediction of the interaction parameter for small values of $\alpha$. This is improved upon by finding a nonlinear relation for $\chi$ that can approximate $\chi$ over a wide range of $\alpha$ using a Taylor series of the form $\chi=z_{\infty} \alpha+\sum_{n=2}^{\infty} C_{n} \alpha^{n}$. Truncating this at $n=3$ gives $\chi=z_{\infty} \alpha+C_{2} \alpha^{2}+C_{3} \alpha^{3}$.

For small values of the interaction parameter, the non-linear $\chi$ reduces to $\chi=z_{\infty} \alpha$, where $z_{\infty}$ is an effective coordination number that can be extracted from the number of intermolecular contacts of an infinitely long chain in an athermal melt (i.e., $\alpha=0$ ) [36]. The average number of intermolecular contacts in an athermal melt should vary as [14]

$$
z(N)=z_{\infty}\left(1+\frac{(6 / \pi)^{3 / 2}}{\bar{N}^{1 / 2}}+O\left(\bar{N}^{-1}\right)\right)
$$


where using $\bar{N}=a^{6} \rho_{0}^{2} N$ gives

$$
z(N)=z_{\infty}\left(1+\frac{(6 / \pi)^{3 / 2}}{a^{6} \rho_{0}^{2} N^{1 / 2}}+\frac{\delta}{N}\right) .
$$

To find the value of $z_{\infty}$, the Morse calibration fits Equation 3.4 to data for the number of intermolecular contacts, collected from simulations run at chain lengths of $N=20,30,40$, 60, 100 and 200. The simulations are run athermally, setting the interaction parameter to $\alpha=0$. Figure 3.2 shows the fit for $c=1,2,3$, and 5 .
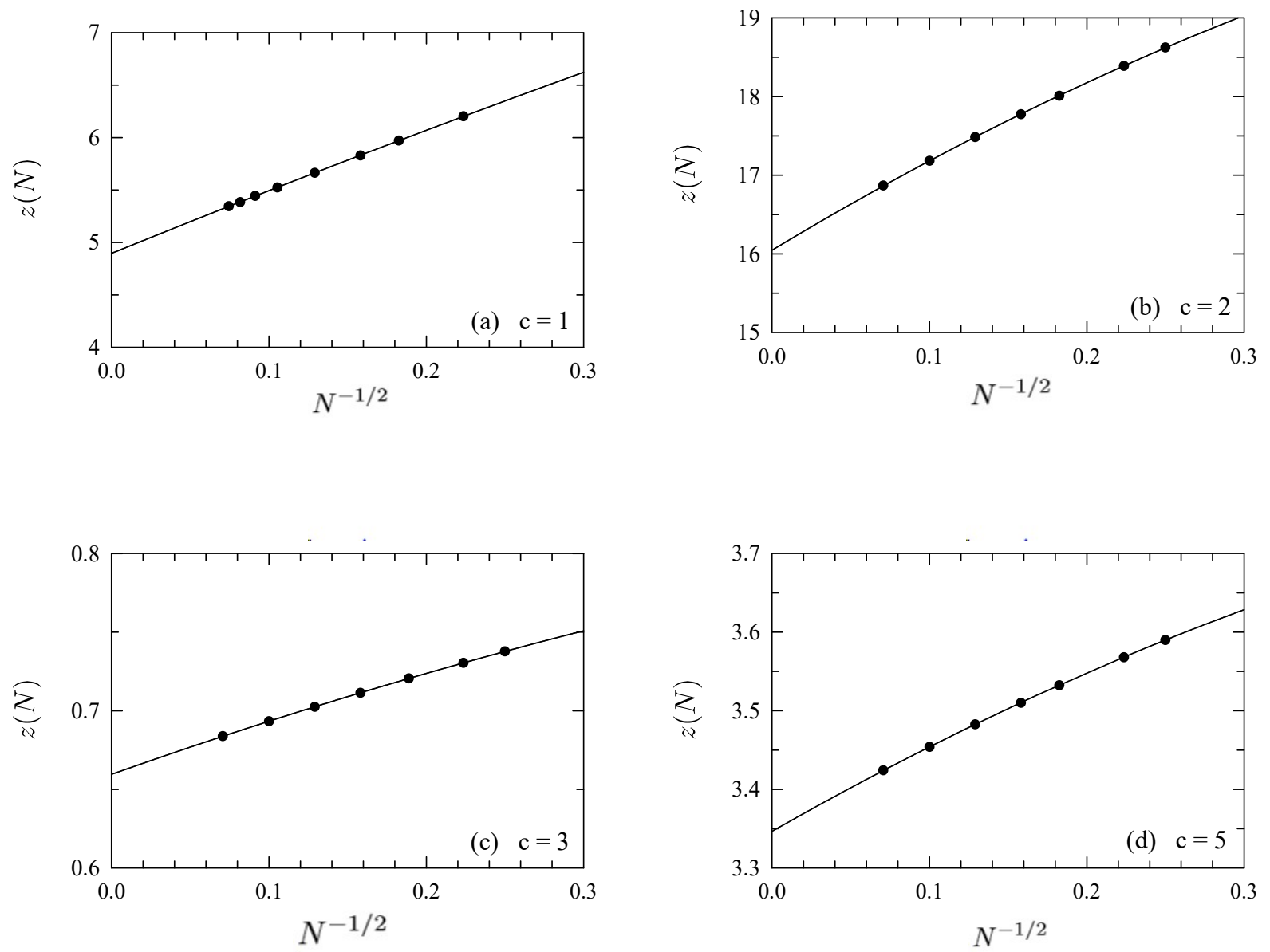

Figure 3.2: Fits of the number of intermolecular contacts used to determine $z_{\infty}$ for $c=1$, 2,3 , and 5 . The symbols are from the lattice simulations and the lines are the fits.

Figure 3.2 (a) shows that with infinitely long chains there will be $z_{\infty}=4.90$ inter- 
molecular contacts on average, for the model with $c=1$ monomer per site. Figure 3.2 (b) show the fit for the model with $c=2$ monomers per site, from which we obtain $z_{\infty}=16.1$ an increase of 11.2. This increase occurs because as we increase the number of nearestneighbour sites from 12 to 25, we would expect the number of intermolecular contacts to increase by $25-12=13$ minus some amount because of the collapsed chain crowding out monomers from other polymers. Figure 3.2 (c) shows the calibration for the model with $c=3$ monomers per site, which gives $z_{\infty}=1.48$. This model has a $z_{\infty}$ that is much smaller than the values from the previous $c=1$ and 2 models. This makes sense, because for the models with more than two monomers per site, the nearest-neighbour interactions have been turned off and only interactions between monomers on the same site remain. This means that there are only two monomers in range to interact with each monomer. For the model with $c=5$ monomers per site, we would expect that the number of intermolecular contacts would increase from the $c=3$ model by slightly less than $5-3=2$. This is exactly what we see with $z_{\infty}=3.34$, giving an increase of $3.34-1.48=1.86$.

\begin{tabular}{||cc||}
\hline$c$ & $z_{\infty}$ \\
\hline \hline 1 & 4.90 \\
\hline 2 & 16.1 \\
\hline 3 & 1.48 \\
\hline 5 & 3.34 \\
\hline
\end{tabular}

Table 3.2: $z_{\infty}$ values for the four models with different numbers of monomers per site, $c$.

A linear $\chi$ can be defined as above, but to have an accurate $\chi$ near the order-disorder transition, we need a $\chi$ that works for higher values of $\alpha$. The Morse calibration finds a non-linear $\chi$ by fitting to results for the diblock disordered-state structure function, $S(k)$, from ROL theory. The fit we use has parameters $C_{2}$ and $C_{3}$ and is of the form

$$
\chi(\alpha)=z_{\infty} \alpha+C_{2} \alpha^{2}+C_{3} \alpha^{3} .
$$

We use the Monte Carlo simulations to calculate the peaks in the structure function over a range in $\alpha$, and then fit the results to predictions of the peak heights from ROL over a range in $\chi N$ to estimate the coefficients $C_{2}$ and $C_{3}$. However, our simulations take place on a finite size lattice so the structure functions are discrete. To find the peaks in the structure function from the simulations, we need to smooth the discrete results. To find the corresponding continuous structure functions for an infinite system, the simulation results are fit to the Leibler structure functions derived from RPA calculations [12]: 


$$
S(k)=\frac{N}{F\left(k^{2} a^{2} N / 6\right)-2 \chi N},
$$

where

$$
F(x)=\frac{g(1, x)}{g(f, x) f(1-f, x)-0.25[g(1, x)-g(f, x)-f(1-f, x)]^{2}},
$$

and $g(f, x)$ is the Debye function from Appendix B. We fit Equation 3.7 to the structure function data generated from the Monte Carlo simulations and extract the peak heights. The simulations were performed with chains of length $N=30,40,60$, and 100 and with $c=1,2,3$, and 5 monomers per site. The only exceptions were for $c=1$, which had $N=90$ instead of $N=100$, and $c=3$, which had $N=28$ in place of $N=30$. This was done to allow the simulations to be run at the correct density, $\rho_{0}$, for the given $N$ and $L$. For demonstration, the fits for chain lengths of $N=30$ for the models with $c=2$ and 5 are shown in figure 3.3 (a) and (b), respectively.
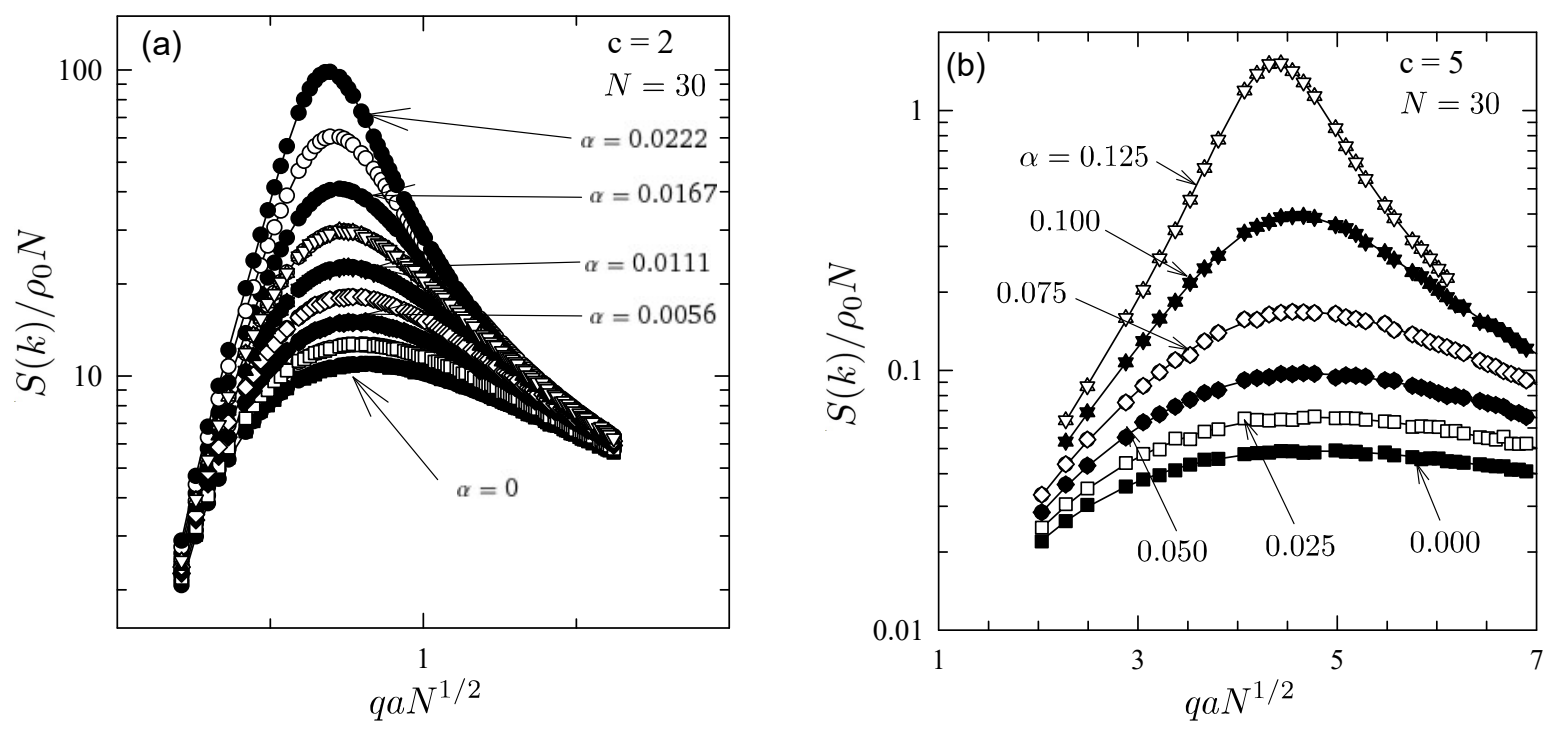

Figure 3.3: Leibler function fits for $N=30$ with $c=2$ and 5

This is repeated for the other chain lengths and the extracted peaks are fit to the predictions from ROL by adjusting $\chi$. For the simulations with $c=1$ monomer per site, the simulations are run at four different chain lengths, $N=30,40,60$, and 90 , and a number of different values of the interaction parameter, $\alpha$. The peak heights of the structure function are extracted by fitting the Leibler function and subsequently fitted to the corresponding 
ROL prediction by adjusting $\chi(\alpha)$. because we do this with a number of different chain lengths this fit works for a wide range of $\alpha$. For the model with $c=1$ monomer per site, the associated $\bar{N}$ values are $135,180,271$, and 451 . This is then repeated for the other three models with $c=2,3$, and 5 using the parameters in Table 3.3.

\begin{tabular}{||cccc||}
\hline$c$ & $N$ & $N$ & $\alpha N$ \\
\hline \hline 1 & $30,40,60,90$ & $135,180,271,406$ & $0.17,0.33,0.5,0.67,0.83,1,1.17,1.33,1.5, \ldots, 3.33$ \\
\hline 2 & $30,40,60,100$ & $372,496744,1240$ & $0.083,0.17,0.25,0.33,0.42,0.58,0.67,0.75,0.83$ \\
\hline 3 & $28,40,60,100$ & $678,968,1452,2420$ & $1,2,3,4,5,6,7,8,9$ \\
\hline 5 & $30,40,60,100$ & $1797,2396,3594,5990$ & $0.75,1.5,2.25,3,3.3,3.75,4.08,4.208$ \\
\hline
\end{tabular}

Table 3.3: A list of all the parameters for the simulations used to calibrate $\chi(\alpha)$.

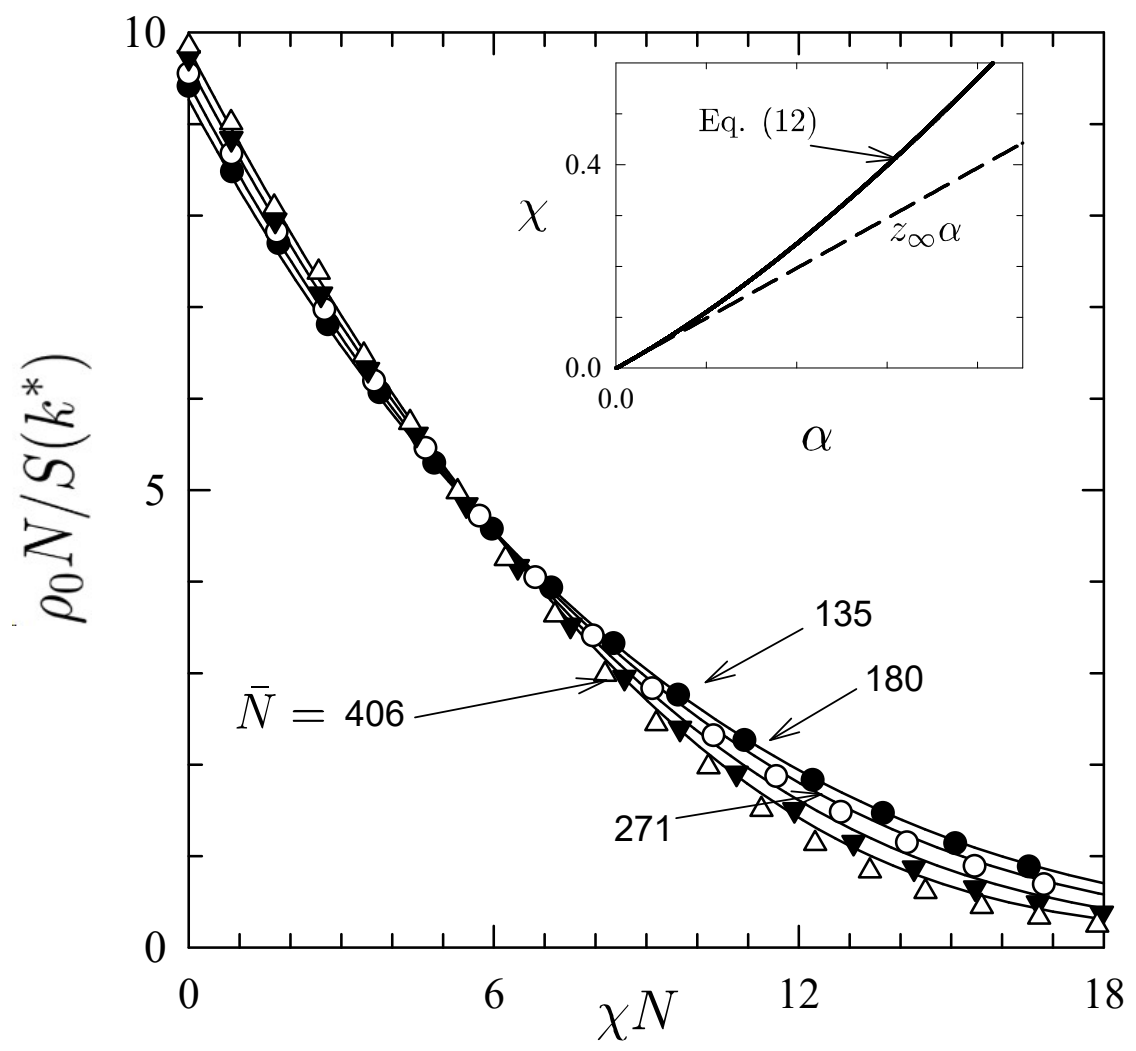

Figure 3.4: Peaks in structure functions for $c=1$. Symbols are from the lattice simulations and curves are ROL predictions. The fit to ROL predictions for $\bar{N}=135,180,271$, and 406 give values of $C_{2}=33.6$ and $C_{3}=-74$. 


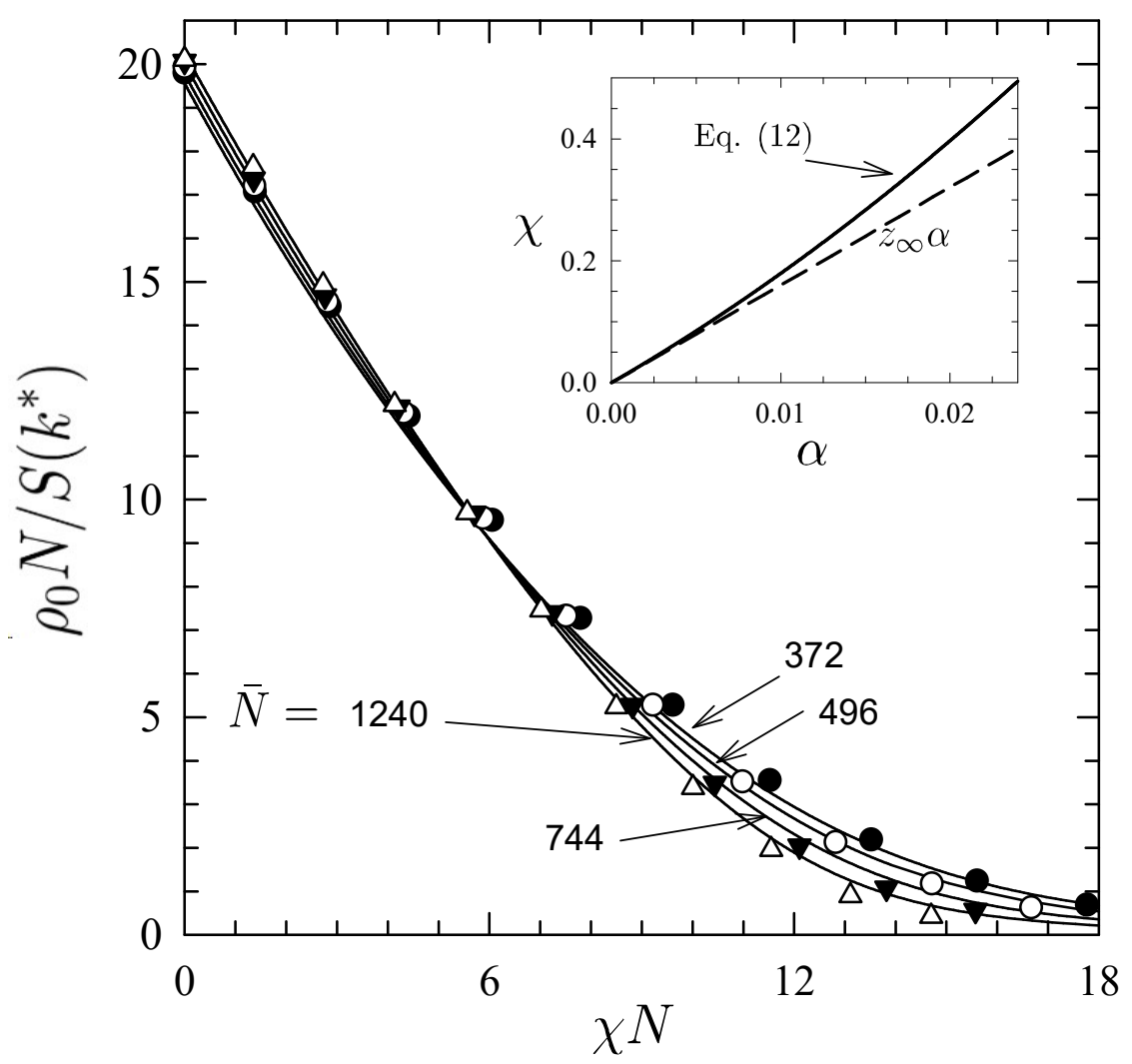

Figure 3.5: Peaks in structure functions for $c=2$. Symbols are from the lattice simulations and curves are ROL predictions. The fit to ROL predictions for $\bar{N}=372,496744$, and 1240 give values of $C_{2}=208$ and $C_{3}=-63$. 


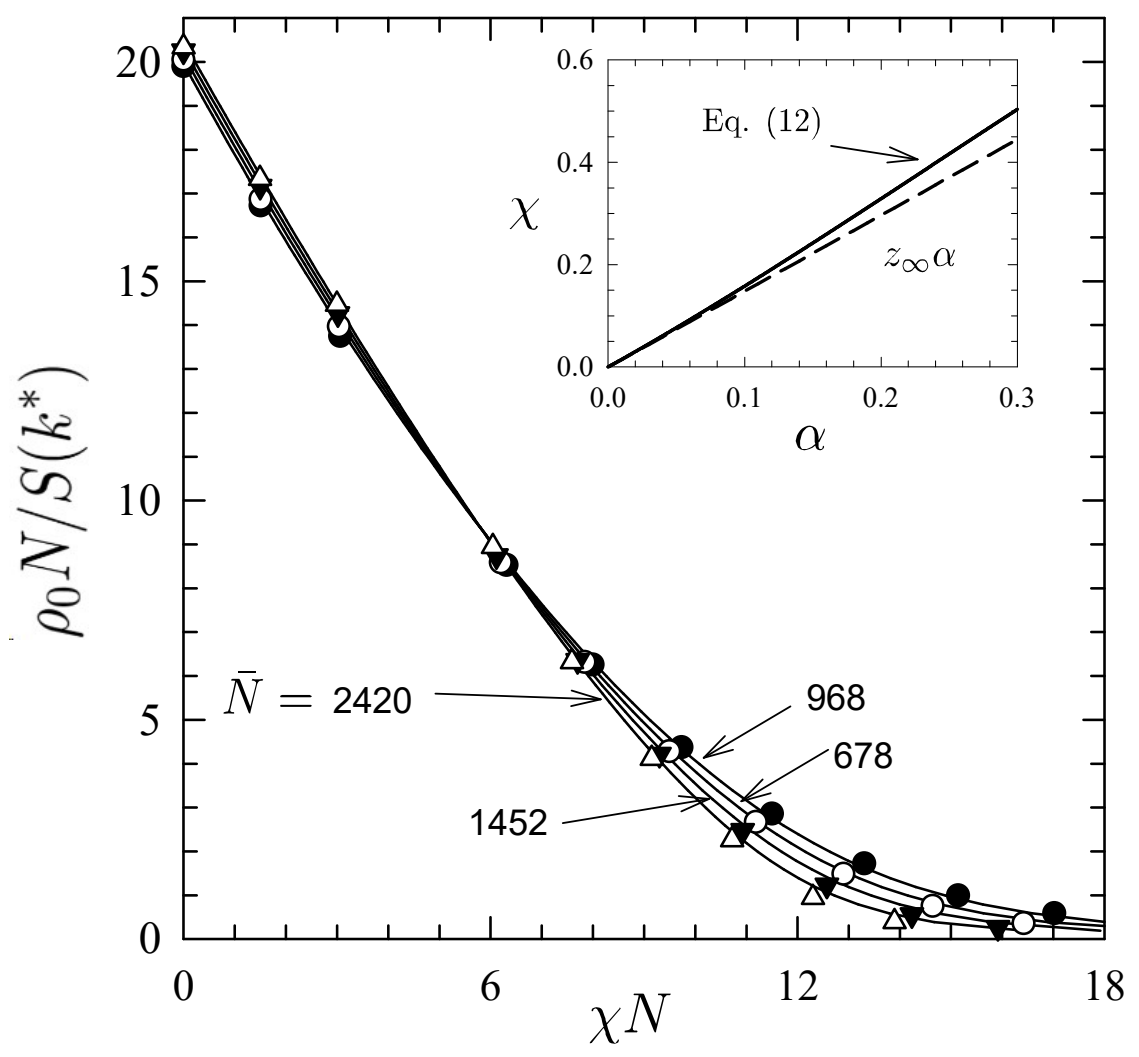

Figure 3.6: Peaks in structure functions for $c=3$. Symbols are from the lattice simulations and curves are ROL predictions. The fit to ROL predictions for $\bar{N}=678,968,1452$, and 2420 give values of $C_{2}=0.68$ and $C_{3}=-0.17$. 


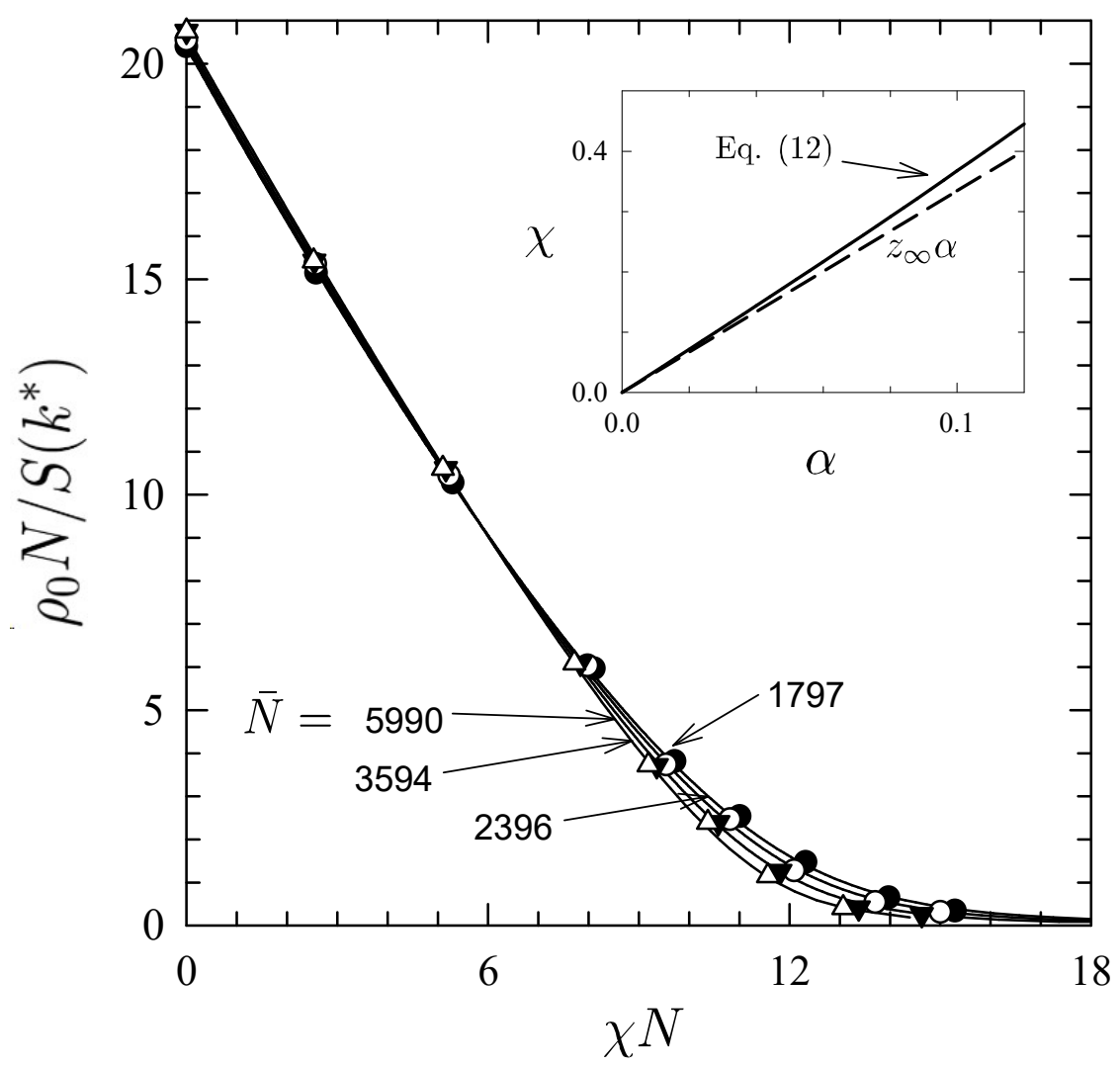

Figure 3.7: Peaks in structure functions for $c=5$. Symbols are from the lattice simulations and curves are ROL predictions. The fit to ROL predictions for $\bar{N}=5990,3594,2396$, and 1797 give values of $C_{2}=4.15$ and $C_{3}=-8.5$.

In the top corners of Figures 3.4, 3.5, 3.6, and 3.7, are comparisons between the linear $\chi$ and the non-linear $\chi$. The calibrated parameters for Equation 3.5 are summarized in Table 3.4.

\begin{tabular}{||cccc||}
\hline$c$ & $z_{\infty}$ & $C_{2}$ & $C_{3}$ \\
\hline \hline 1 & 4.90 & 33.6 & -74 \\
\hline 2 & 16.1 & 208 & -63 \\
\hline 3 & 1.48 & 0.68 & -0.17 \\
\hline 5 & 3.34 & 4.15 & -8.5 \\
\hline
\end{tabular}

Table 3.4: Parameters for Equation 3.5 relating $\chi$ and $\alpha$, calculated by a fit of the Monte Carlo simulation data to predictions from ROL theory. 
With this, we have calibrated the lattice model. This will let use translate from the lattice model parameters, $\alpha$ and $N$, to the universal parameters $\chi$ and $\bar{N}$. Thus, we are now able to make predictions that are universal and that can be applied to any system.

These predictions of the ODT for symmetric diblocks are used to calibrate $\chi$ for diblocks in experiments. Commonly in experiments, the mean-field prediction is used to do this calibration. However, this neglects the fluctuations, polydispersity, and compositional asymmetry present in all experimental systems. Including these factors in the calibration increases the accuracy of the calibration and might help resolve previously noted contradictions between different methods of determining $\chi$ [28]. To include these factors this work adds a multiplicative correction factor to the universal ODT curve, which will be discussed in the next chapter. 


\section{Chapter 4}

\section{Order-Disorder Transition}

The order-disorder transition (ODT) is the point in the phase diagram where the stable phase changes between the disordered phase and an ordered morphology. The specific ordered morphology at the ODT is dependent on the composition, $f$, and invariant polymerization index, $\bar{N}$. For this work, we will be focusing on $f=0.5$, so all the polymer melts will form an ordered lamellar phase. The mean-field theory prediction for the ODT of symmetric diblocks is $(\chi N)_{O D T}=10.495$. However, this prediction is not accurate for melts with finite length polymers because the fluctuations in the melt act to push the ODT to higher values of $\chi N$. A prediction that includes these fluctuation corrections is the universal curve

$$
(\chi N)_{O D T}=10.495+41 \bar{N}^{-2 / 3}+123 \bar{N}^{-0.56},
$$

The first term is the standard mean-field theory prediction. Added to this is the correction $41 \bar{N}^{-2 / 3}$ from Fredrickson-Helfand theory [15]. The last term was determined by fitting results from multiple simulation methods to ROL predictions [22]. In order to test the accuracy of our lattice model calibration, we can determine the ODTs of a series of symmetric monodisperse diblock copolymer melts and compare them to the universal curve.

\subsection{Monodisperse Order-Disorder Transition}

The ODT is the point where the lamellar and disordered phases coexist and in our lattice models it is signified by an abrupt change in the average number of A-B contacts $\left\langle n_{A B}\right\rangle$ $[32,35,37,38]$. Therefore, by running a series of parallel tempering replicas that span 
the lamellar and disordered phases, we can collect statistics for $\left\langle n_{A B}\right\rangle$ and detect the transition. However, the difference in free energy between the two phases becomes small near the ODT, leading to a large time scale for the unfavorable phase to convert to the global minimum. Therefore, simulations started in a disordered configuration and run just above the ODT may not order over the length of our simulation run. As a result, this metastability will lead to some inaccuracies in our determination of $(\chi N)_{O D T}$. To estimate these errors, we can run two sets of simulations. In the first set, the melts start from a lamellar configuration and will underestimate the ODT, while the second set starts from disorder and will overestimate the ODT. By plotting the loop formed by overlaying the two simulation runs as in Figure 4.1, the range bracketing the ODT can be determined. The value of $(\chi N)_{O D T}$ is taken to be the center of this range.
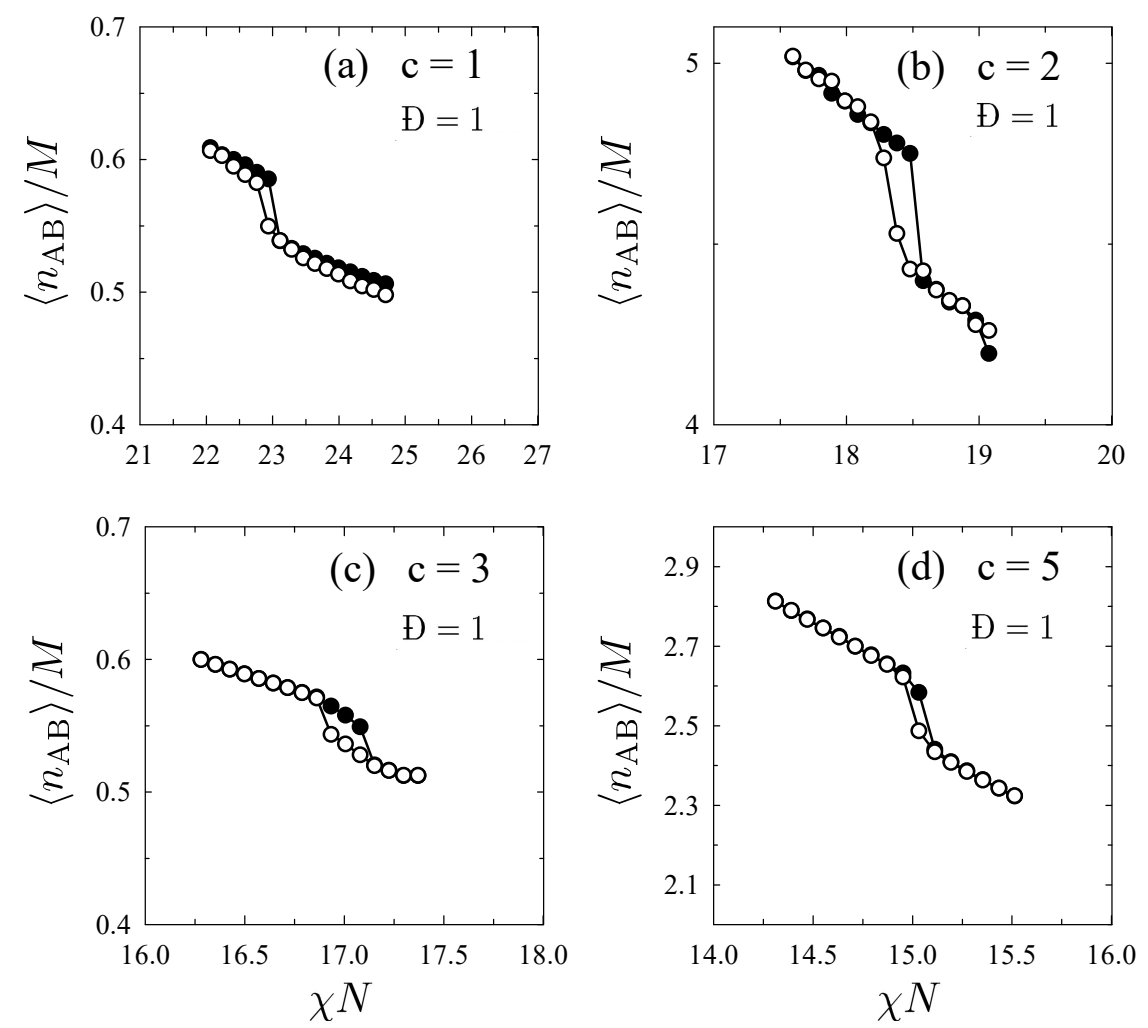

Figure 4.1: Average number of $\mathrm{AB}$ contacts, $\left\langle n_{A B}\right\rangle$, from parallel-tempering simulations of monodisperse diblock copolymers with $N=50$ and with $c=1,2,3$, and 5 monomers per site, respectively. Open symbols are started from a lamellar configuration and closed symbols are started from disorder. the box size $L$ is picked so that there are approximately 3 periods in the box. 
Figure 4.2 compares the order-disorder transitions from our calibrated simulations to the universal curve given by Equation 4.1.

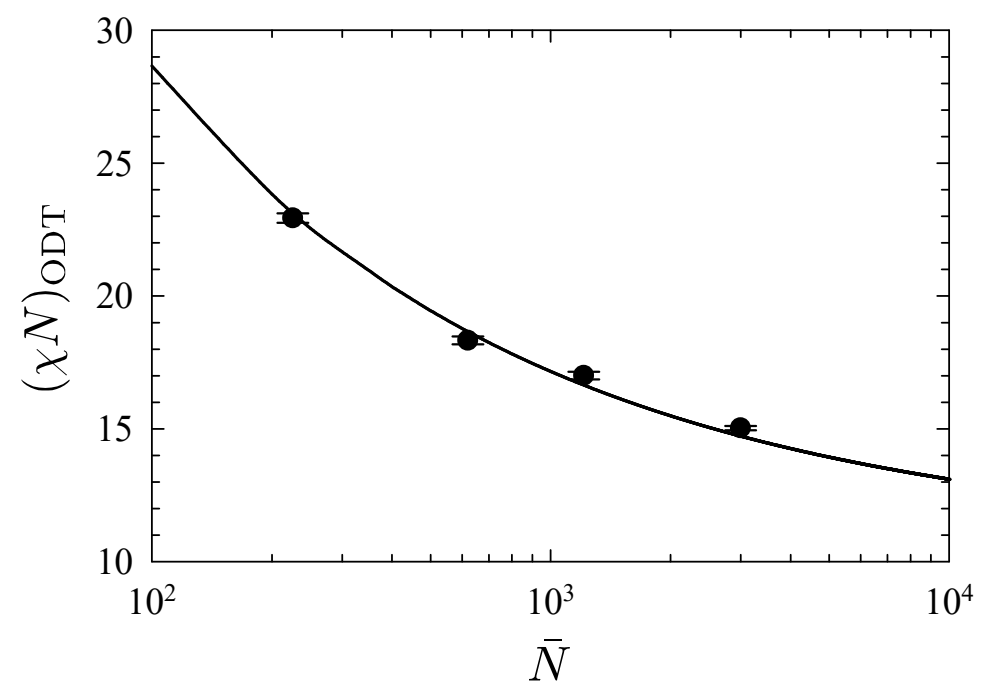

Figure 4.2: Location of the ODT, $(\chi N)_{O D T}$, as a function of the invariant polymerization index, $\bar{N}$. The curve is the universal curve, Equation 4.1, and the points are the results from our lattice simulation with $N=50$ and $f=0.5$. The uncertainty from the lattice model predictions are the error bars.

The excellent agreement between our data and universal curve confirms that the calibration is working well. In the next section, we will use the lattice model to make predictions for how the universal curve changes with polydispersity.

\subsection{Polydisperse Correction to the ODT}

One popular method for calibrating $\chi$ in experiments is to match the ODT to some theoretical prediction[19-39]. While experimentalists endeavor to keep the level of polydispersity minimal, the reality of synthesizing diblocks means that $Đ \approx 1.1$ is typical. Although relatively low, this level of polydispersity was still shown to have an important effect on the calibration of PI-PLA diblocks [37]. Our aim in this section is to calculate a polydispersity correction to the monodisperse universal curve displayed in figure 4.2 to facilitate accurate calibration of experiments. We will limit our investigation to polydispersity indexes of $Đ=1.05$ and 1.1 . 
In general the polydispersity index alone does not capture all the change in behavior due to the polydispersity of the melts $[8,9]$. However, for the low range of $Đ$ considered here, the universal curve for polydispersity is well approximated by a correction proportional to the polydispersity index, and, we may ignore the higher order-moments of the distribution [39]. Hence, we expect the expression for the polydisperse ODT to take the form

$$
(\chi N)_{O D T}^{\text {poly }}=(\chi N)_{O D T}^{\text {mono }}(1+\kappa(Đ-1)),
$$

where $\kappa$ is a coefficient that depends on $\bar{N}$ and is determined by plotting $(\chi N)_{O D T}$ as a function of . As such, we must first determine $(\chi N)_{O D T}$ at $Đ=1.05$ and 1.10 for each of our $c=1,2,3$, and 5 models. This is shown in figure 4.3 , where we plot $\left\langle n_{A B}\right\rangle$ vs. $(\chi N)$ and determine $(\chi N)_{O D T}$ from the centers of the metastability loops. The values are summarized in Table 4.1, along with the monodisperse data. Note that because polydispersity increases the periodicity of the lamellar phase [32, 39, 40], we increase the size of the simulation box to keep the number of periods the same. 

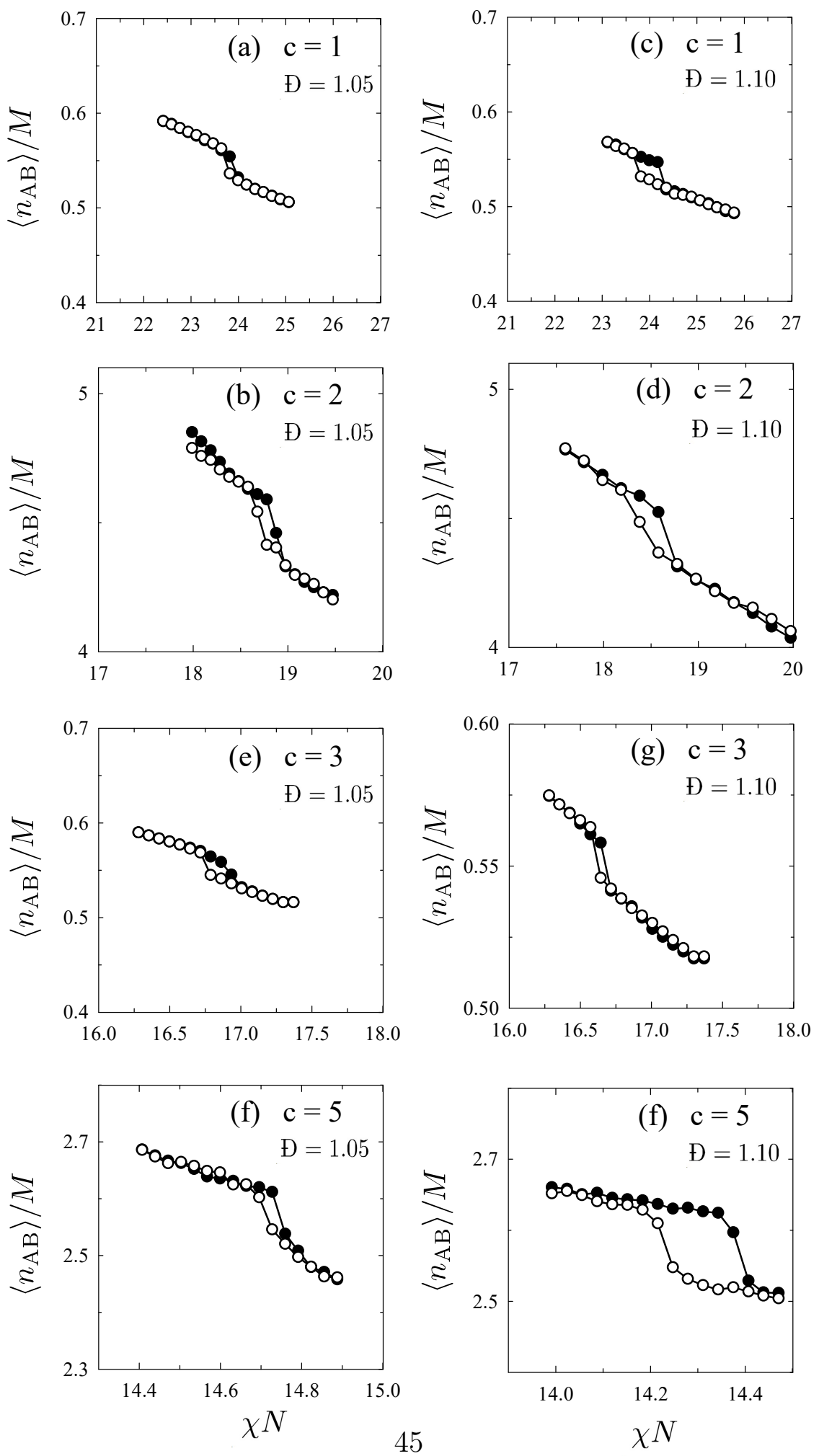

Figure 4.3: Metastability loops in the average number of intermolecular contacts per monomer $\left\langle n_{A B}\right\rangle / M$ over a range of the universal interaction strengths, $\chi N$. The melts are polydisperse with index $\mathrm{Ð}$, average chain length $\langle N\rangle=50$ and $c=1,2,3$, and 5 monomers per site respectively. Open symbols are started from a lamellar configuration and closed symbols are started from disorder. 


\begin{tabular}{||ccccc||}
\hline$c$ & $\bar{N}$ & $\begin{array}{c}(\chi N)_{O D T} \\
\mathrm{D}=1\end{array}$ & $\begin{array}{c}(\chi N)_{O D T} \\
\mathrm{D}=1.05\end{array}$ & $\begin{array}{c}(\chi N)_{O D T} \\
\mathrm{D}=1.1\end{array}$ \\
\hline \hline 1 & 226 & 22.9 & 23.8 & 23.9 \\
\hline 2 & 620 & 18.3 & 18.7 & 18.5 \\
\hline 3 & 1210 & 17.0 & 16.9 & 16.6 \\
\hline 5 & 2995 & 15.0 & 14.7 & 14.3 \\
\hline
\end{tabular}

Table 4.1: $(\chi N)_{O D T}$ for melts with three different polydispersities of $Đ=1,1.05$, and 1.10 for our models with $c=1,2,3$, and 5 monomers per site.

Each subplot in Figure 4.4 corresponds to a fit of the data in Table 4.1 to Equation 4.2, for (a) $c=1$, (b) $c=2,(\mathrm{c}) c=3$, and (d) $c=5$, where the slopes of the linear fits give the values of $\kappa$ that are summarized in Table 4.2 .
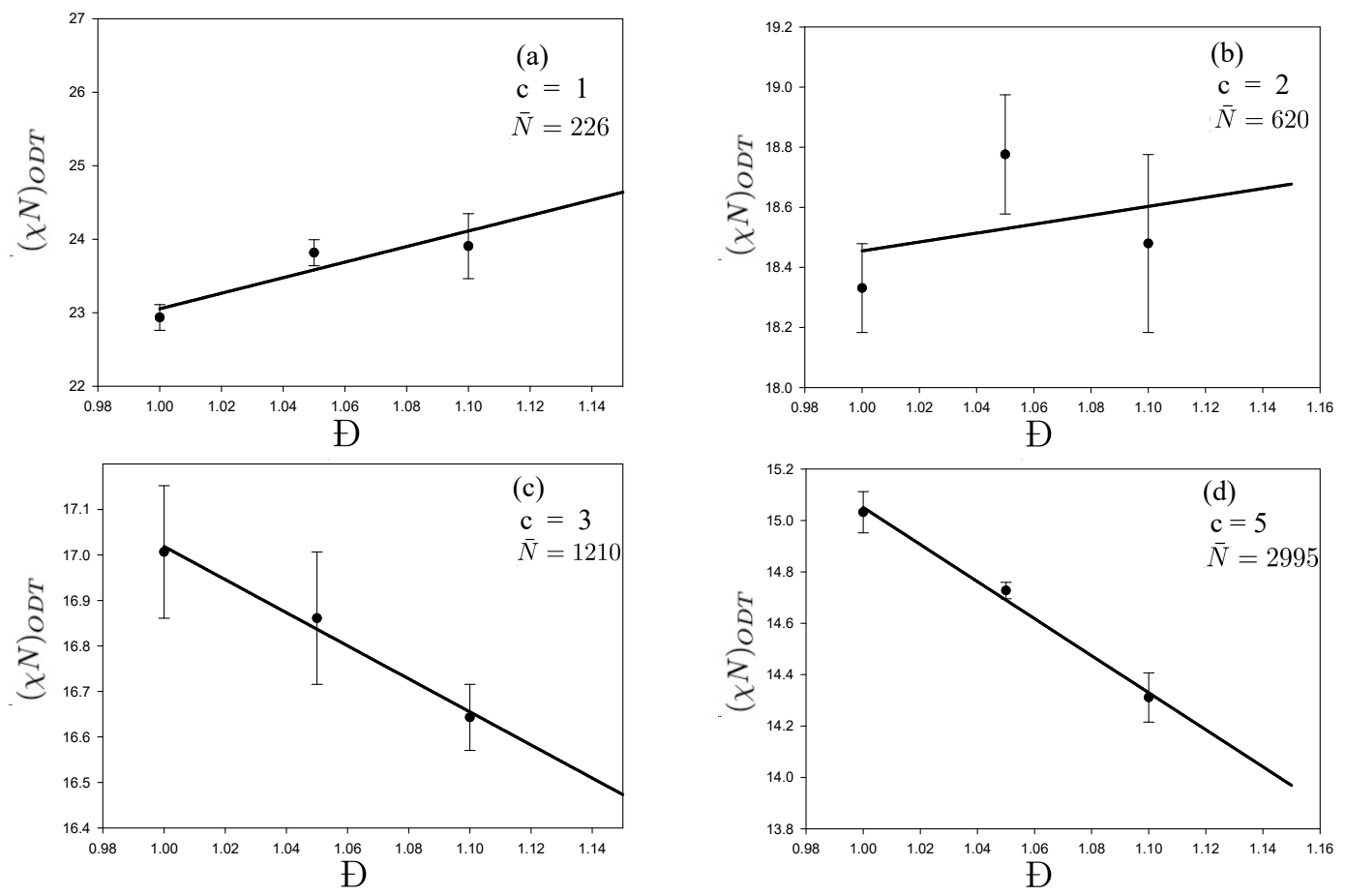

Figure 4.4: Fits of the polydispersity correction used to find $\kappa$. Symbols are data from the lattice simulations. The error bars are the widths of the metastability loops and the lines are the fits to Equation 4.2. 
Interestingly Figure 4.4 shows that as the value of $\bar{N}$ increases, the sign of the correction changes from positive to negative between $\bar{N}=620$ and $\bar{N}=1210$.

\begin{tabular}{||ccc||}
\hline$c$ & $\bar{N}$ & $\kappa$ \\
\hline \hline 1 & 226 & 0.459 \\
\hline 2 & 620 & 0.080 \\
\hline 3 & 1210 & -0.213 \\
\hline 5 & 2995 & -0.479 \\
\hline
\end{tabular}

Table 4.2: Linear polydispersity correction, $\kappa$, for the four models with different numbers of monomers per site, $c$.

To find an accurate value of the function $\kappa(\bar{N})$, we need to know its asymptotic behavior for large $\bar{N}$. In the limit of large $\bar{N}$, mean-field theory is accurate and, so the RPA analysis of a polydisperse melt provides the value of $\kappa$. RPA gives the structure function as

$$
S(k)=\frac{N}{F(k)-2 \chi N},
$$

where the function $F(k)$ is simplified for $f=0.5$ to

$$
F(k)=\bar{g}_{1}(0.5, x)-\frac{1}{4} \bar{g}_{1}(1, x),
$$

and the number-average Debye function, $\bar{g}_{1}$, is given by

$$
\bar{g}_{1}(\bar{f}, \bar{x})=2\left[\bar{f} \bar{x}+(1+\bar{f} \bar{x} K)^{-K^{-1}}-1\right] / \bar{x}^{2} .
$$

Where $K=1 /(\mathrm{\Xi}-1)$ and $x=k^{2} a^{2} N_{n} / 6$. The transition at the value of $f=0.5$ is secondorder, and second-order transitions have their ODT at the spinodal. Therefore, by finding the point where the structure function diverges, we will also find the ODT. Looking at Equation 4.3, we can see that $S(k)$ diverges if $F(k)=2 \chi N$, giving the ODT for $f=0.5$ as

$$
(\chi N)_{O D T}=\frac{1}{2} F\left(k_{L a m}\right),
$$

where the value of $k_{\text {Lam }}$ can be found by minimizing $F(k)$. Doing this over a range of $Đ$ gives the dependence of the ODT on polydispersity in the mean-field theory. We can then extract $\kappa$ from a linear fit to $(\chi N)_{O D T}$ vs $\mathrm{Ð}$, as shown in Figure 4.5. 


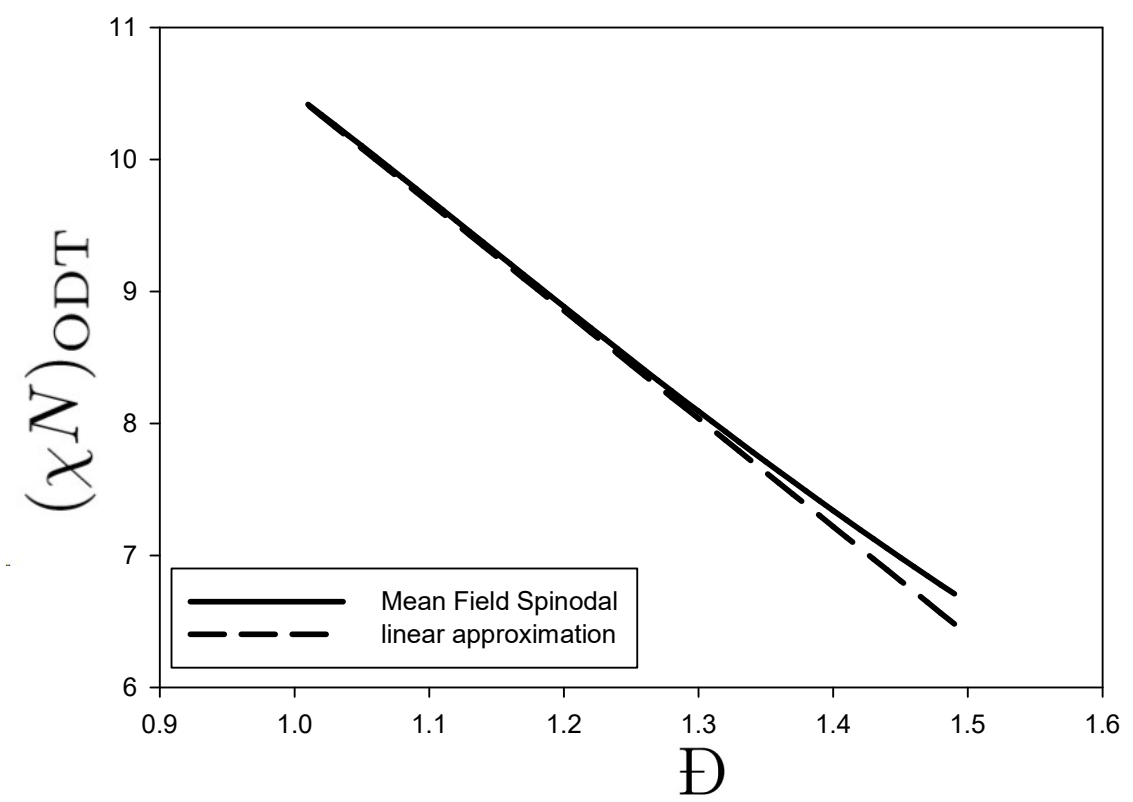

Figure 4.5: Fitting Equation 4.2 to the prediction of $(\chi N)_{O D T}$ from RPA gives the value of $\kappa$ for $\bar{N}=\infty$ as $\kappa=-0.77$.

With this we get the polydispersity correction $\kappa=-0.77$ for mean-field theory. Note that $(\chi N)_{O D T}$ is close to the linear approximation over the relevant range of $\mathrm{\Xi}<1.1$.

To find the function $\kappa(\bar{N})$, we fit a smooth curve of form

$$
\kappa(\bar{N})=-0.77+C \bar{N}^{B},
$$

to the $\kappa$ values summarized in Table 4.2 constrained to the mean-field value, which ensures the correct behavior in the limit of $\bar{N}=\infty$. The fitting parameters were found to take the values $B=-0.49$ and $C=17.5$, as shown in figure 4.6. 


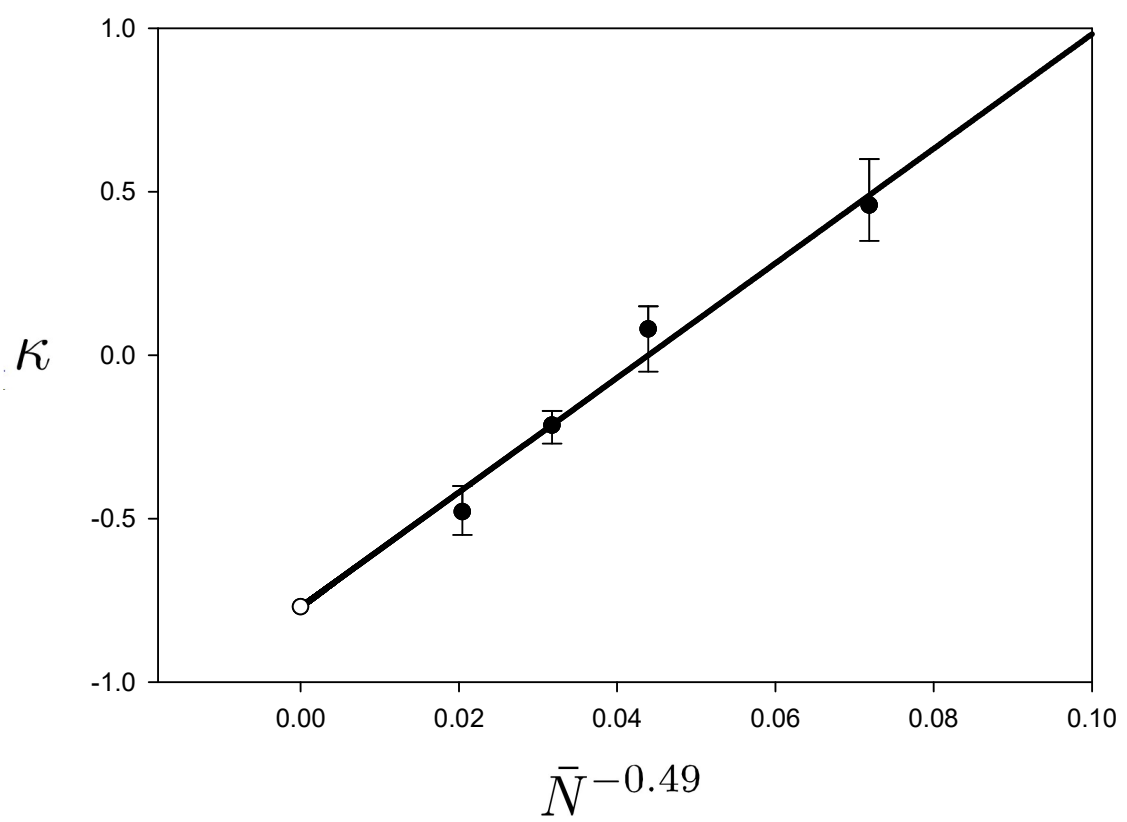

Figure 4.6: Dependence of the linear correction for polydispersity, $\kappa$, on the invariant polymerization index, $\bar{N}$. Closed circles are from our simulation data, the open circle is from mean-field theory, and the line is Equation 4.7 with $B=-0.49$ and $C=17.5$.

Together with Equation 4.2, this expression for $\kappa$ gives a correction to the universal curve for polydispersity, allowing for a more accurate calibration of $\chi$ in experimental systems. However, there is another correction to the universal curve that is also important in the calibration.

\subsection{Compositional Asymmetry Correction to the ODT}

To improve the calibration of experimental systems, we need to correct for the small amount compositional asymmetry that always exists in real systems. This can be achieved in the same way as we did for polydisperse systems. The necessary data, summarized in Table 4.3, already exists in the literature from the Morse group [31]. We begin by adding an asymmetry correction to Equation 4.2, of the form

$$
(\chi N)_{O D T}=(\chi N)_{O D T}^{i \text { deal }}\left(1+\kappa(\boxplus-1)+\gamma\left(f-\frac{1}{2}\right)^{2}\right) .
$$


This correction must be of quadratic order due to the symmetry of the phase diagram around $f=0.5$. The $\bar{N}$ dependence of $\gamma$ is determined by fitting to simulations of compositionally asymmetric diblocks for $\bar{N}=480$ and 1920 from the Morse group, summarized in Table 4.3, and constraining the fit to the known mean-field value of $\gamma=6.7$ from Matsen and Bates [11].

\begin{tabular}{||ccc||}
\hline & $\begin{array}{c}(\chi N)_{O D T} \\
f\end{array}$ & $(\underline{N}=4)_{O D T}$ \\
$\bar{N}=1920$ \\
\hline \hline 0.5 & 19.57 & 15.61 \\
\hline 0.4688 & 19.76 & 15.63 \\
\hline 0.4375 & 20.76 & 16.14 \\
\hline 0.4062 & 22.41 & 17.16 \\
\hline
\end{tabular}

Table 4.3: ODTs for $\bar{N}=480$ and 1920 and various compositions $f$, with $Đ=1$ from Ghasimakbari, T.; Morse, [31].

For a particular $\bar{N}, \gamma$ is determined by plotting $(\chi N)_{O D T}$ as a function of $(f-1 / 2)^{2}$ and extracting $\gamma$ from a fit of Equation 4.8 to the data. Figure 4.7 displays these fits using the Morse group data summarized in Table 4.3.
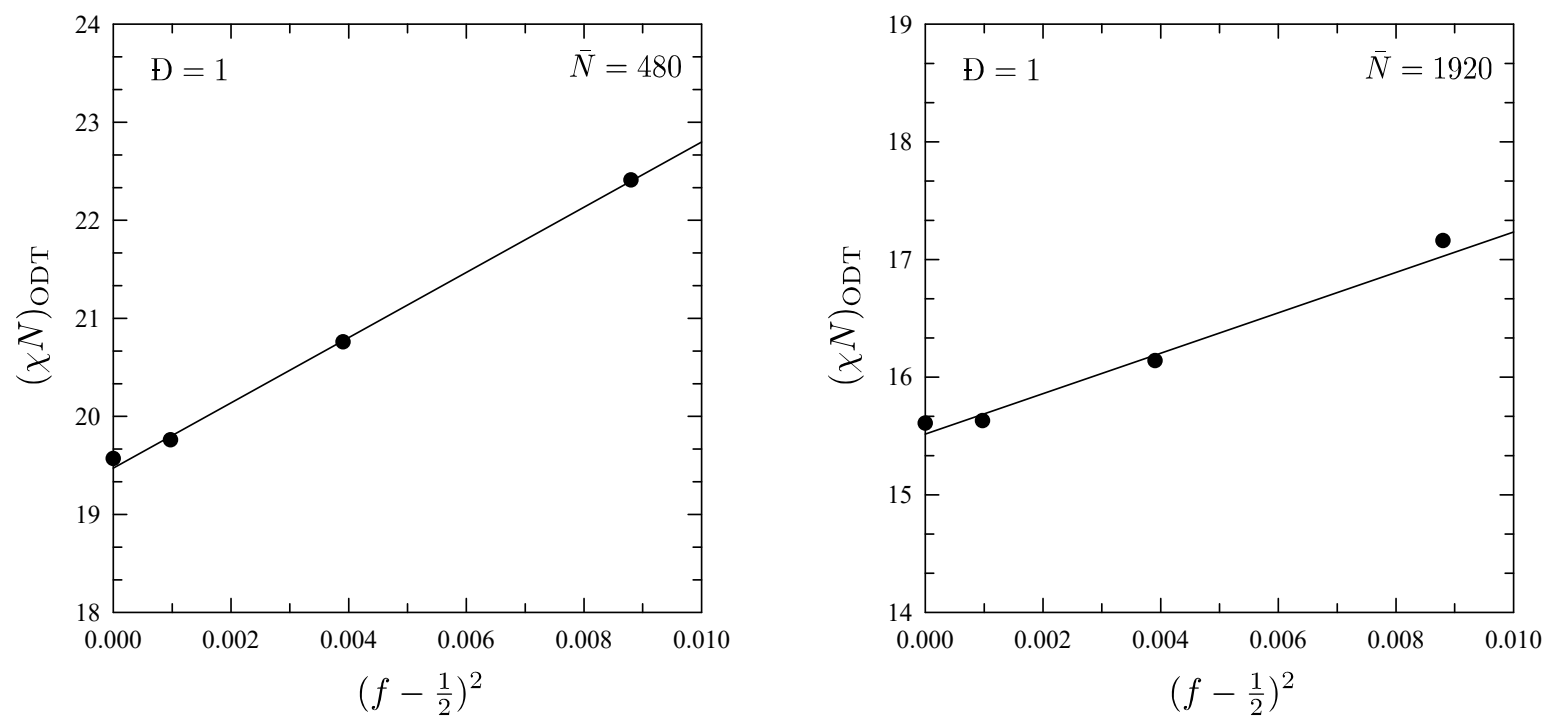

Figure 4.7: ODTs for different values of compositional asymmetry. Points are from the data in Table 4.3, and lines are fits to Equation 4.8. The parameter $\gamma$ is given by the slope of the lines. 
We can see in Figure 4.7 that much like $\kappa, \gamma$ is a parameter that depends on $\bar{N}$. This dependence of $\gamma$ on $\bar{N}$ is fit to the functional form $\gamma(\bar{N})=6.7+C_{1} \bar{N}^{C_{2}}$, as shown in figure 4.8 where the fitting parameters where determined to be $C_{1}=500$ and $C_{2}=-0.63$

$$
\gamma(\bar{N})=6.7+500 \bar{N}^{-0.63}
$$

This fit is has two parameters and it is fit to two data points this accounts for the large degree of agreement between the fit and data in Figure 4.8.

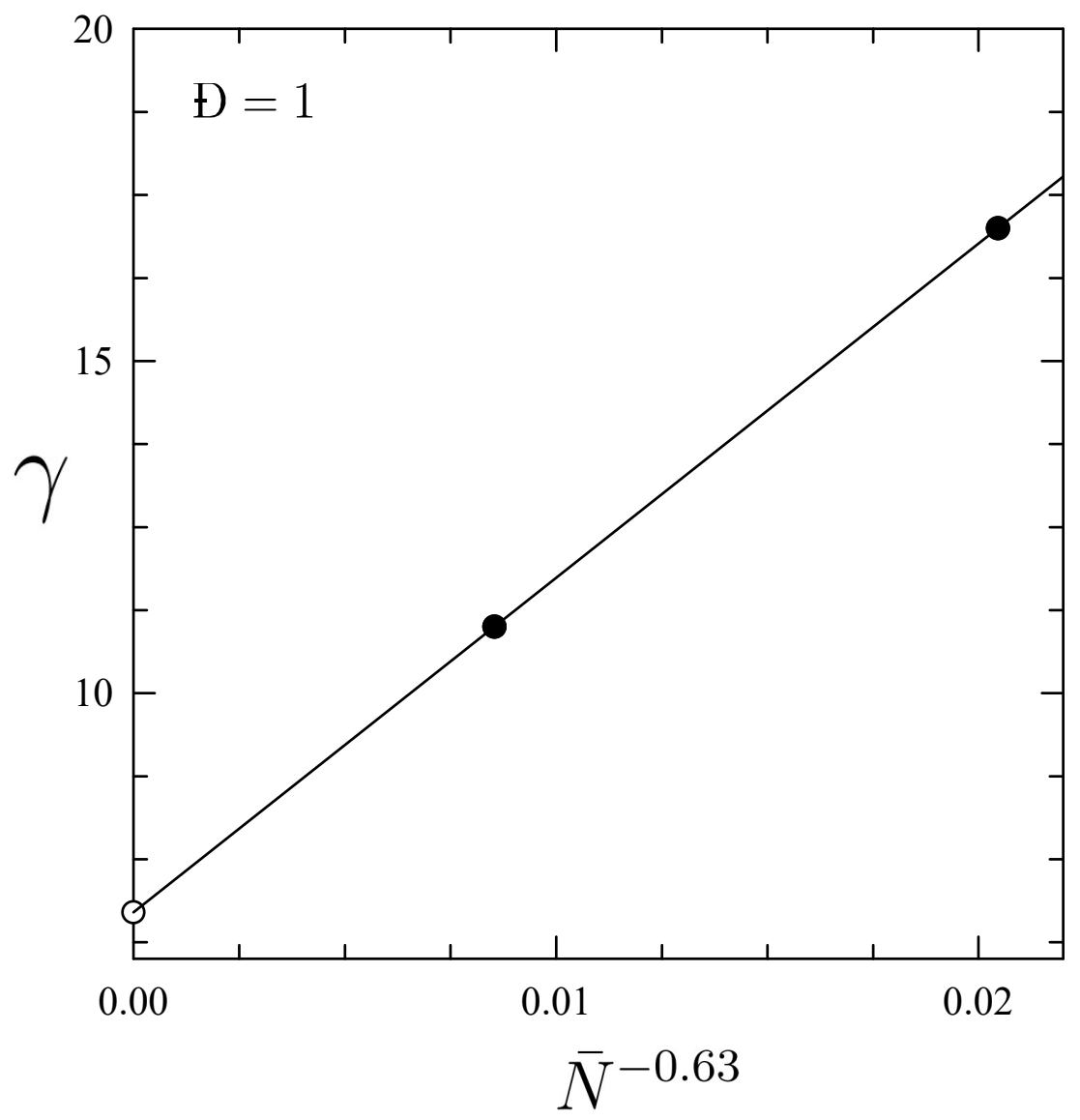

Figure 4.8: Dependence of the linear correction for polydispersity on the invariant polymerization index $\bar{N}$. Closed symbols are from the Morse group data [31], while the open symbol is a mean-field value from Matsen and Bates [11]

We can now accurately predict the ODT of diblock copolymer melts with a small degree 
of polydispersity and compositional asymmetry. In the next section, calibrations of the Flory-Huggins $\chi$ parameter using the new ODT predictions will be calculated for a number of different diblock chemistries using experimental data. These calibrations improve upon ones done using the less accurate mean-field predictions for the ODT, $(\chi N)_{O D T}=10.495$. 


\section{Chapter 5}

\section{Calibration of Experimental Systems}

In experimental systems, the parameters commonly reported are the molecular weight, $M_{n}$, the composition, $f_{A}$, and the polydispersity index, Đ. In addition we also utilise, the known literature values for the $A$ and $B$ blocks of segment density, $\rho_{A}$ and $\rho_{B}$, and end-to-end distance of the polymer, $R_{0}$. In order to proceed with the calibration we require the invariant polymerization index, $\bar{N}=a^{6} \rho_{0}^{2} N$, where $a$ is the segment length, $\rho_{0}$ is the standard segment density and $\mathrm{N}$ is the degree of polymerisation. We start by determining $\mathrm{N}$. The size of a segment is defined by the standard segment volume, $\rho_{0}^{-1}=118 \AA^{3}$, and the mass of a segment is the product of its density and volume, $\rho_{A} / \rho_{0}$. By dividing the molecular weight by the mass of a segment, we then have the number of chain segments, $N=\frac{\rho_{0} M_{n}}{\rho_{A}}$, which would be true if we where dealing with A-type homopolymer.

However, finding the number of segments is a little more complicated for diblocks as the two blocks have different densities. As a result, the number of segments in the $\mathrm{A}$ and B blocks must be calculated separately by $N_{\gamma}=\frac{M_{n, \gamma} \rho_{0}}{N_{A \nu} \rho_{\gamma}}$, where the subscript, $\gamma=A$ or $B$, labels quantities specific to the particular blocks, and $N_{A \nu}$ is Avogadro constant. This gives a total diblock polymerization of $N=N_{A}+N_{B}$, and composition $f=\frac{N_{A}}{N}$. By combining the equations for $N$ and $N_{\gamma}$, we can express the total polymerization as

$$
N=\frac{M_{n} \rho_{0}}{N_{A \nu}\left(f \rho_{A}+(1-f) \rho_{B}\right)} .
$$

The other necessary quantity is the statistical segment length, $a$, which can be derived from the average end-to-end distance of the polymer, $R_{0}$. Using Equation 1.5 and known data for $R_{0}[41,42,43]$, we can find the independent statistical segment lengths for the 
two blocks via

$$
a_{\gamma}^{2}=N_{A \nu} \frac{R_{0}^{2}}{M_{n}} \frac{\rho_{\gamma}}{\rho_{0}} .
$$

To find the average statistical segment length, we average the two blocks, giving

$$
a^{2}=f_{A} a_{A}^{2}+\left(1-f_{A}\right) a_{B}^{2}
$$

With Equations 5.1 and 5.3 we can now calculate the invariant polymerization index, $\bar{N}=a^{6} \rho_{0}^{2} N$. Table 5.1 reports the calibrated quantities for a number of different chemical species.

\begin{tabular}{cccc} 
Chemistry & $R_{0}^{2} / M_{n}\left(\frac{\AA^{2} m o l}{g}\right)$ & $\rho\left(\frac{m g}{\mathrm{~cm}^{3}}\right)$ & $a(\AA)$ \\
\hline \hline PE[41] & 1.250 & 0.784 & 8.35 \\
PEE[41] & 0.507 & 0.807 & 5.39 \\
PEP[41] & 0.834 & 0.790 & 6.84 \\
PCHE[41] & 0.323 & 0.920 & 4.60 \\
PI[41] & 0.625 & 0.830 & 6.07 \\
PS[41] & 0.434 & 0.969 & 5.47 \\
PEO[41] & 0.805 & 1.064 & 7.80 \\
PMMA[41] & 0.425 & 1.130 & 5.84 \\
PLA[42, 43] & 0.605 & 1.152 & 7.03 \\
PDMS[41] & 0.457 & 0.895 & 5.39 \\
\hline
\end{tabular}

Table 5.1: Molecular data used in calculating the number of segments and the segment mass. $R_{0}^{2} / M_{n}$ is the literature value of how radius of gyration scales with chain length. $\rho$ is the mass density from the literature and $a$ is the statistical segment length calculated from Equation 5.3.

With this data we can now find the relationship between $\chi$ and the temperature in the experiments. This is done by taking the locations of the order-disorder transition from experiments in terms of temperature, $\mathrm{T}$, and mapping them onto predictions from theory expressed in terms of $\chi$. To compare the importance of the relative corrections, in the predictions of $(\chi N)_{O D T}$, the calibration of $\chi$ will be performed using order-disorder transitions calculated using four different theoretical results. The first and least accurate prediction of the ODT is from mean-field theory,

$$
(\chi N)_{O D T}=10.495
$$


Secondly, there is an additional correction from Fredrickson-Helfand theory that accounts for some of the concentration fluctuations [15], giving

$$
(\chi N)_{O D T}=10.495+41 \bar{N}^{-1 / 3} .
$$

The third result is the universal curve that was fit to monodisperse symmetric simulations calibrated to ROL theory [22],

$$
(\chi N)_{O D T}=10.495+41 \bar{N}^{-1 / 3}+123 \bar{N}^{-0.56} .
$$

The final prediction is Equation 4.8 from Chapter 4 (repeated here for clarity),

$$
(\chi N)_{O D T}=(\chi N)_{O D T}^{\text {ideal }}\left(1+\kappa(\boxplus-1)+\gamma\left(f-\frac{1}{2}\right)^{2}\right),
$$

which includes our corrections for polydispersity and compositional asymmetry. In order to proceed with the calibration, we use the common functional form

$$
\chi(T)=\frac{A}{T}+B,
$$

to express $\chi$ in terms of temperature in the experiments. Adjusting the fitting parameters, $\mathrm{A}$ and $\mathrm{B}$, then allows us to map the experimental ODTs, $T_{O D T}$, onto the predictions for $(\chi N)_{O D T}$. To demonstrate this calibration, we will use data for PCHE-PMMA from Kennemur, Bates, and Hillmyer [59], presented in Table 5.2.

\begin{tabular}{cccc}
\hline$N$ & $\mathrm{D}$ & $f$ & $T_{O D T}$ \\
\hline \hline 137 & 1.10 & 0.45 & $605 \mathrm{~K}$ \\
127 & 1.11 & 0.58 & $598 \mathrm{~K}$ \\
76 & 1.12 & 0.54 & $477 \mathrm{~K}$ \\
65 & 1.11 & 0.46 & $446 \mathrm{~K}$ \\
\hline
\end{tabular}

Table 5.2: Tabulation of data for PCHE-PMMA for degree of polymerization $N$ calculated with Equation 5.1, polydispersity $\mathrm{Ð}$, composition $f$, and the experimental temperance of the order-disorder transition $T_{O D T}$ from [59].

Using the data from this Table 5.2, we can predict the theoretical order-disorder transition, starting with the mean-field prediction of Equation 5.4. This gives the value $\chi_{O D T}=10.495 / N$ for the vertical axis of Figure 5.1. We then compare this to the observed order-disorder transition in the experiments $T_{O D T}$ by putting inverse temperature 
on the horizontal axis. Drawing a straight line through each of the data sets, we can find $A$ and $B$. The quality of the fit justifies our choice of functional form in Equation 5.8. As a result, we are able to extract the fitting parameters, $\mathrm{A}$ and $\mathrm{B}$, from the slope and intercept, respectively, thereby calibrating $\chi$ for the experiments.

To improve this calibration we can use a more accurate prediction for the theoretical order-disorder transition from Fredrickson-Helfand theory, Equation 5.5. Dividing this by $N$ gives the value of $\chi_{O D T}$. This prediction includes a fluctuation correction to the ODT that is dependent on $\bar{N}$, which is responsible for the difference between the first two calibrations. We can see in Figure 5.1 that improving the accuracy of the calibration has a large effect on the value of $\chi$. This shows the importance of improving the accuracy of the calculation of the ODT used in the calibration. We can next improve the calibration by using a more accurate prediction of the ODT from the universal curve. Equation 5.6. We can see in Figure 5.1 this calibration adds another important correction to the prediction of $\chi$. Finally we can improve the calibration by not just including the dependence on $\bar{N}$ but also the dependence on $Đ$ and $f$. This is done by using the prediction of the ODT from this work, Equation 5.7. 


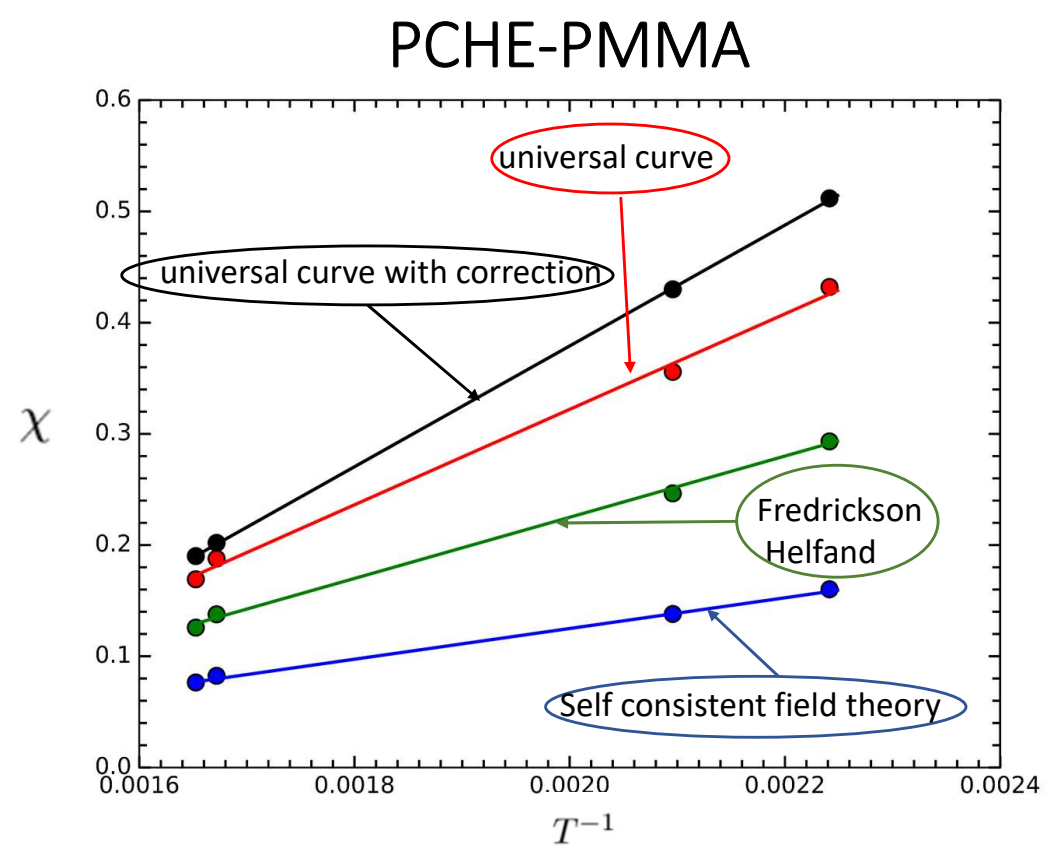

Figure 5.1: Plot of the calibration of $\chi$ for PCHE-PMMA using data from Kennemur, Bates, and Hillmyer [59]. The calibration based on mean field ODT Equation 5.8 gives $A=138$ and $B=0.151$. The calibration based on Fredrickson Helfand ODT Equation 5.6 gives $A=275$ and $B=0.325$. The calibration based on Universal curve ODT Equation 5.7 gives $A=429$ and $B=0.536$. The calibration based on this works correction ODT Equation 5.8 gives $A=543$ and $B=0.707$.

Its important to compare the final calibration, based on the corrected universal curve, in Figure 5.1 to the calibration based on the mean field theory prediction of $(\chi N)_{O D T}=$ 10.495, as this is the typical calibration used in the literature. This demonstrates the size of the correction to the calibrations over common methods and the importance of including all the corrections to the ODT. Also do this calibration for 18 other diblock chemistries. In Figure 5.4, this same calibration is carried out. 

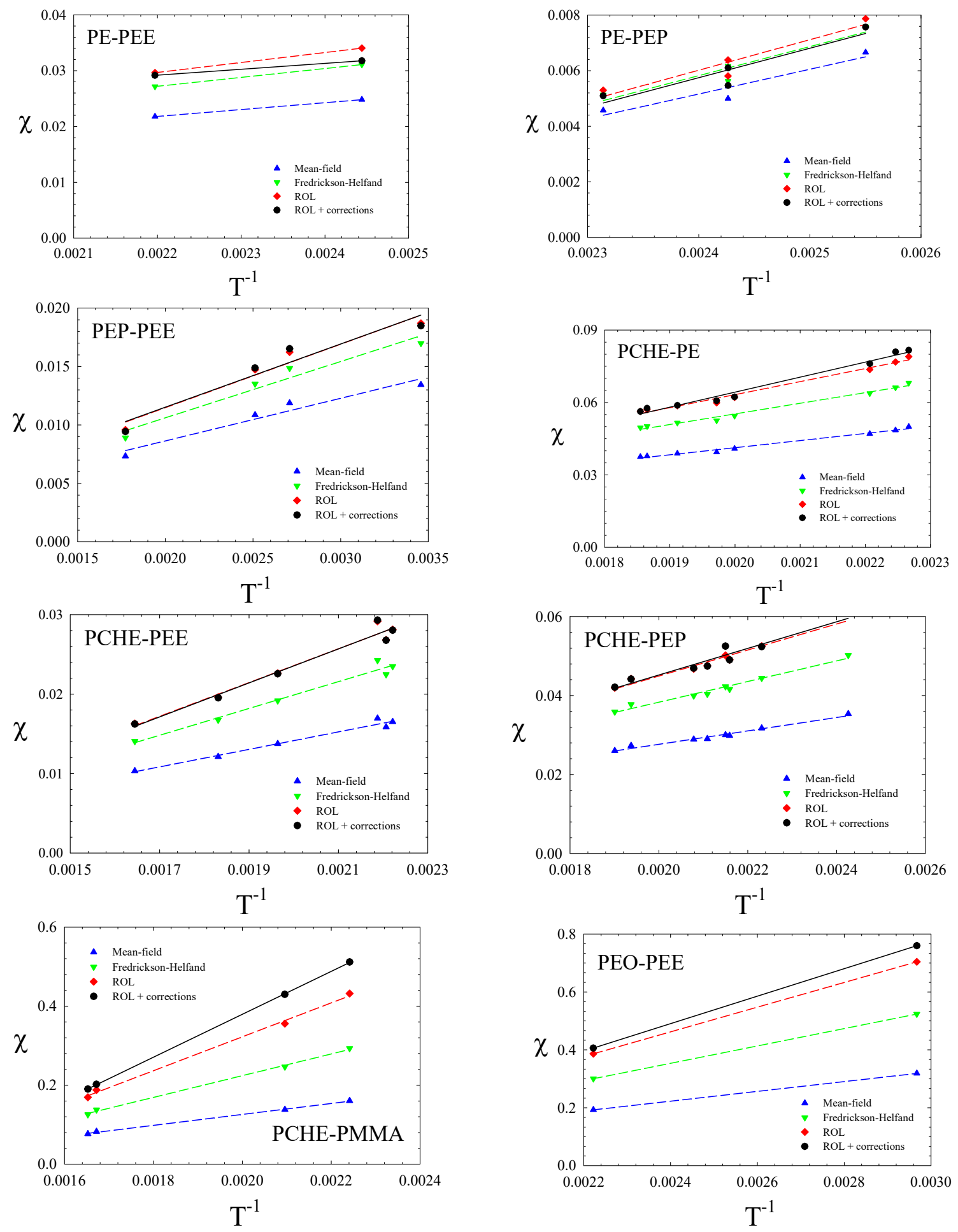

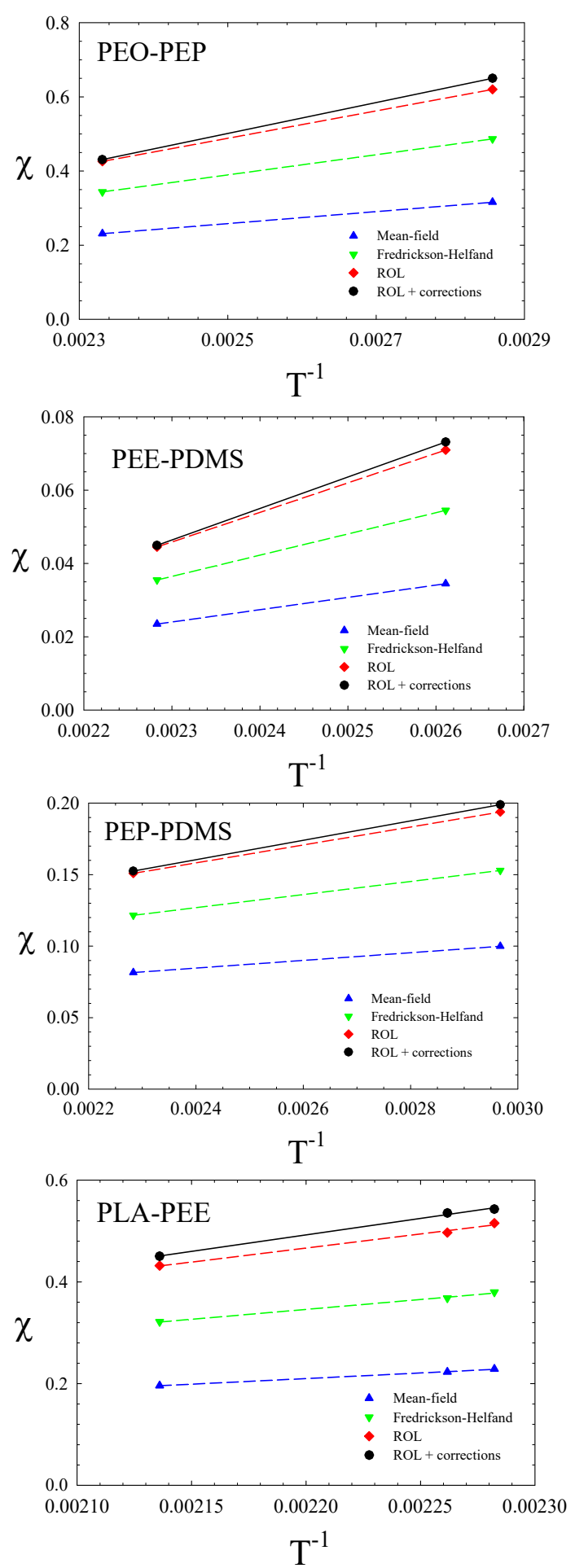
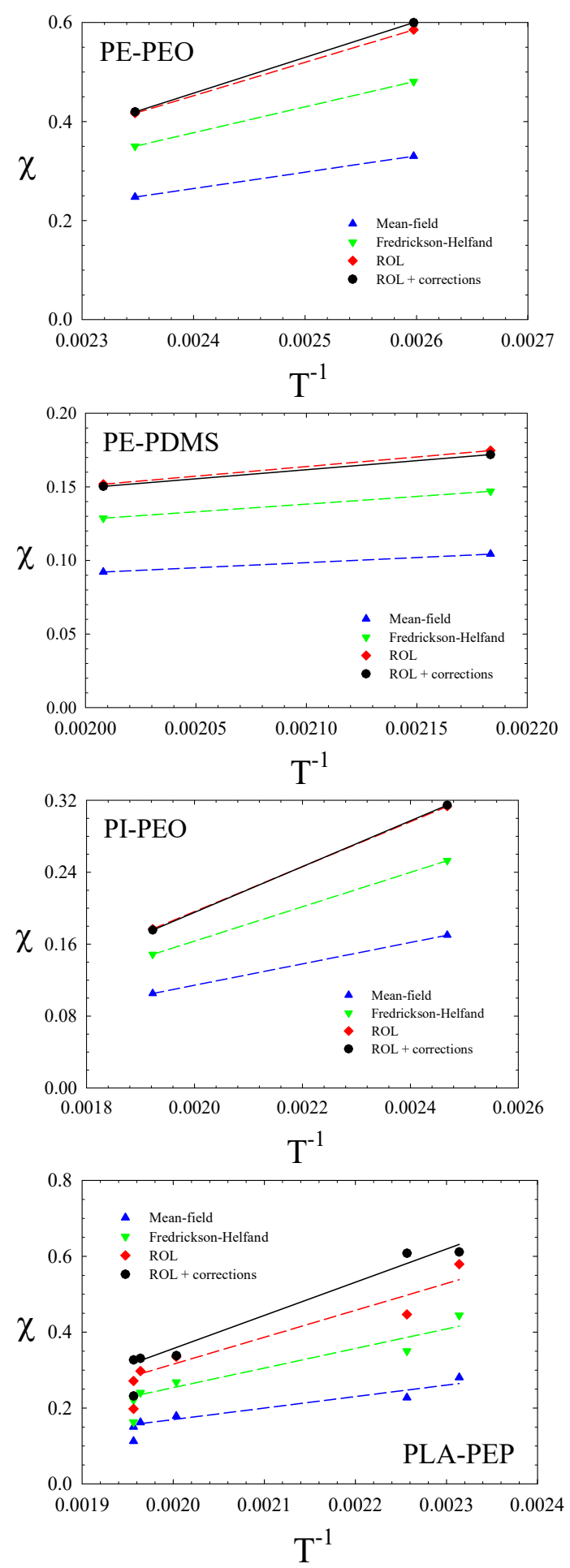

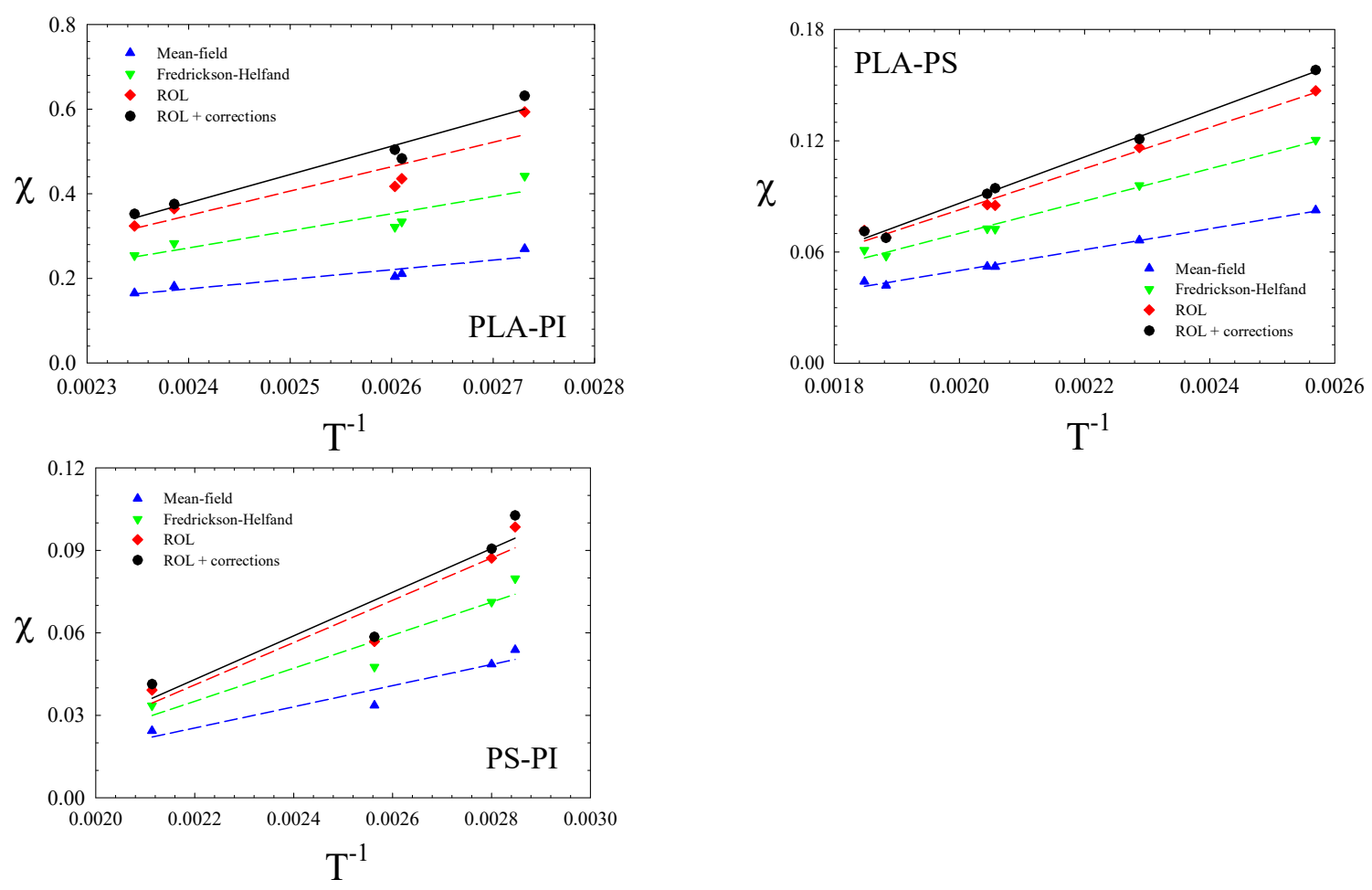

Figure 5.4: Analogous plots to Figure 5.1 for 19 different experimental systems. Calibration of $\chi$ for 19 experimental systems.

In all cases, using a more accurate prediction of $(\chi N)_{O D T}$ has greatly improved the calibration of $\chi$ compared to fitting to the mean-field value of $(\chi N)_{O D T}=10.495$. The fluctuation corrections for finite $\bar{N}$ make a large change to the ODT in the experimental regime, thus affecting the calibration. In the calibrations of PLA-PEP, PLA-PI and PLAPS there is a large correction from including polydispersity and compositional asymmetry. PLA-PEP has an increase of $100 \%$ over the mean-field result, $20 \%$ of which results from the polydispersity and asymmetry corrections. This illustrates the importance of using more accurate predictions for $(\chi N)_{O D T}$.

In some of the calibrations the corrections for polydispersity and compositional asymmetry are small compared to the other corrections. For example, this can be seen in the calibrations of PCHE-PEE and PCHE-PEP where the, polydispersity index is very low $(Đ=1.02)$, and the composition is almost symmetric $(f \approx 0.5)$, leading to a correspondingly small correction from these quantities.

Interestingly, in some of the calibrations where polydispersity is larger i.e. $(Đ \approx 1.10)$ 
the correction for polydispersity is small compared to the other corrections. This is because for $\bar{N}=590$, the correction factor for polydispersity, $\kappa$, is approximately zero, as seen in Figure 4.6. This small value of $\kappa$ means that the polydispersity has a very weak effect on the ODT and thus on the calibration. For example, in the calibration of PI-PEO, despite the data points having polydispersity indexes of $\mathrm{\Xi}=1.06$ and $\mathrm{\Xi}=1.10$, the calibration was not changed significantly by including polydispersity. This effect is most pronounced for values of $\bar{N} \approx 600$.

All the newly calibrated $\chi$ parameters accounting for polydispersity and compositional asymmetry are tabulated in Table 5.3. However, a complete record of the different calibrations to all four predictions considered are presented in Appendix A.

\begin{tabular}{ccc}
\hline chemical pair & $A$ & $B \times 10^{3}$ \\
\hline \hline PE-PEP [61] & 10.60 & -19.68 \\
PEP-PEE [61] & 5.40 & 0.72 \\
PCHE-PEE [54] & 21.6 & -19.2 \\
PE-PEE [61] & 10.5 & 6.0 \\
PCHE-PEP [54] & 33.7 & -22.2 \\
PEE-PDMS [55] & 86.0 & -151.3 \\
PCHE-PE [54] & 62.1 & -60.0 \\
PS-PI [26, 30, 64] & 79.3 & -131.5 \\
PLA-PS [57] & 125 & -163 \\
PE-PDMS [55] & 123 & -97 \\
PEP-PDMS [55] & 68 & -2 \\
PI-PEO [30, 64] & 255 & -314 \\
PCHE-PMMA [59] & 543 & -707 \\
PLA-PEE [60] & 647 & -931 \\
PE-PEO [55] & 722 & -1275 \\
PLA-PEP [56] & 875 & -1393 \\
PLA-PI [58] & 669 & -1227 \\
PEO-PEP [55] & 416 & -540 \\
PEO-PEE [55] & 475 & -649 \\
\hline
\end{tabular}

Table 5.3: Tabulation of calibrated $\chi$ values for data from various experimental papers. A and $\mathrm{B}$ are the fitting parameters from Equation 5.4.

These improved estimates of $\chi$ are easy to implement in place of standard calibrations based on the mean-field prediction of $(\chi N)_{O D T}=10.495$, because they do not require the 
addition of any information that is not provided as standard in the experimental literature, like the polydispersity of the independent blocks. Also, they benefit from the fact that unlike the Morse calibration, these calibrations do not require fitting of the peak heights of the disordered-state structure functions to ROL. This simplifies the experiments as they do not require intensity-calibrated scattering function data. Also, our method is able to account for the polydispersity and compositional asymmetry in systems, whereas implementing the Morse calibration would necessitate new ROL predictions for the structure functions including these factors.

A direct comparison between calibrations based on ODTs and the Morse calibration has been performed previously by Arora et al. [63, 30, 65]. This calibration used predictions for symmetric and monodisperse melts, decreasing its accuracy compared to our method. However, it showed that both a direct Morse calibration based on the structure functions and a calibration based upon the ODTs provide similar results under the same assumptions. As a result, the calibrations in this work, which include corrections for both polydispersity and compositional asymmetry, should be capable of replicating the accuracy of a direct Morse calibration of on experimental system without the need for new experiments and more sophisticated ROL calculations.

All the newly calibrated $\chi$ parameters accounting for polydispersity and compositional asymmetry are tabulated in Table 5.3. However, a complete record of the different calibrations to all four predictions considered are presented in Appendix A. 


\section{Chapter 6}

\section{Conclusion}

\subsection{Conclusion}

We greatly improved the estimation of the Flory-Huggins $\chi$ parameter from previous estimates [61, 57, 54] by accounting for fluctuations and adding corrections to the orderdisorder transition (ODT) for polydispersity and compositional asymmetry. Our approach was built upon the common technique of calibrating $\chi$, whereby experimental ODTs of symmetric diblock copolymer melts are mapped onto the mean-field prediction, $(\chi N)_{O D T}=10.495$. This mean-field based approach neglects the fluctuations inherent in all polymers of finite chain length, which can lead to huge inaccuracies in the calibration. For example, a realistic experimental system could have an $\bar{N}=1000$, which would give a prediction of $(\chi N)_{\mathrm{ODT}}=17.0$, from the universal curve, an increase of $60 \%$ from the mean-field ODT. This initial inaccuracy carries through to the final calibration of $\chi$. Furthermore, calibrating experimental ODTs to $(\chi N)_{O D T}=10.495$ neglects the polydispersity of the molecules as well as the fact that diblock copolymers are never perfectly symmetric.

The method used in this work corrects for deviations from the universal curve for the ODT of ideal molecules by introducing a multiplicative correction factor. This factor is obtained by expanding around $Đ=1$ and $f=\frac{1}{2}$ to the lowest non-trivial order, resulting in the expression

$$
(\chi N)_{\mathrm{ODT}}=\left(10.495+41 \bar{N}^{-1 / 3}+123 \bar{N}^{-0.56}\right)\left(1+\kappa(Đ-1)+\gamma\left(f-\frac{1}{2}\right)^{2}\right) .
$$

The two coefficients, $\kappa$ and $\gamma$, both depend on $\bar{N}$. To extract the $\bar{N}$ dependence for the polydispersity correction, simulations of symmetric diblocks were run for three different 
degrees of polydispersity, $Đ=1,1.05$, and 1.10 , giving

$$
\kappa=-0.77+17.5 \bar{N}^{-0.49} \text {. }
$$

The correction for compositional asymmetry was found in a similar way using existing simulations by Ghasimakbari and Morse [31], giving

$$
\gamma=6.7+500 \bar{N}^{-0.63} \text {. }
$$

Using our updated expression for the order-disorder transition, Equation 6.1, we recalibrated the $\chi$ parameter for 19 different chemically-distinct diblock copolymers that were previously calibrated using $(\chi N)_{O D T}=10.495$. This change from calibrations based on mean-field theory to calibrations based on our more accurate prediction of the ODT provided a huge improvement in the estimated $\chi$ values. Nevertheless, the accuracy of the new calibrations will still be limited by the experimental uncertainties in measuring the ODT as well as the molecular weights, densities, segment lengths, and polydispersities. Given the analytical form of Equation 6.1, a direct error analysis of the calibration of $\chi(T)$ could be performed in an attempt to assess the effect of these errors on the calibrations. However, this would require estimates for the various experimental uncertainties, which were not quoted in the literature.

Although our approach for calibrating $\chi$ assumes that it only depends on temperature, $T$, there are some researchers that suggest $\chi$ may also depend on the details of the system such as polydispersity, composition, molecular weight, and the architecture of the polymers. This concern was raised by Maurer et al. [28] when they found a significant discrepancy in $\chi$ values calibrated from diblock copolymer melts compared to binary homopolymer blends. If this was actually true, then every experimental system would need to be calibrated independently and theory would no longer be able to make quantitative predictions. However, previous experimental calibrations of $\chi$ generally involve fits to mean-field theory and they also ignore deviations from an ideal system (i.e., polydispersity and compositional asymmetry). Therefore, it impossible to know the true cause of the discrepancy between different calibrations of $\chi$. On the other hand, in simulations where these issues can be well controlled, the universality between different systems has been well established [22]. We expect the same will be true of experiments once the details of their system are well accounted for.

\subsection{Further Work}

Other possibilities for future work would be to extend the calibration method by considering the impact of higher-order effects on our prediction for the order-disorder transition. 
For example, we could allow for different polydispersities, $\bigoplus_{A}$ and $\bigoplus_{B}$, in the $\mathrm{A}$ and $\mathrm{B}$ blocks of the diblock copolymer; this work assumed $\bigoplus_{A}=\bigoplus_{B}$. Instead of just expanding the correction factor in terms of the total polydispersity, Đ, we would expand in terms of the individual polydispersities of the two blocks, $\mathrm{Ð}_{A}$ and $\bigoplus_{B}$. Naturally, this would require additional simulations where the A block was polydisperse while the B block remained monodisperse. Note that analogous simulations for a monodisperse A block and a polydisperse B block would not be necessary as they would be identical due to the compositional symmetry (i.e., $f=\frac{1}{2}$ ).

Another improvement that could be incorporated into our calibration would be to account for possible differences in statistical segment lengths of the two blocks, referred to as conformational asymmetry. However, this extension to the method would not be straightforward. Running simulations with different values of statistical segment length could be acheived by adding a bending rigidity to one of the components in order to increase its statistical segment length. However, calibrating the resulting simulations would be a problem because there is currently no renormalized one-loop prediction for conformational asymmetry diblocks. If ROL predictions for the disordered-state structure function were produced for conformationally asymmetric diblocks, then this correction could be readily incorporated into our approach. Nevertheless, the effect on the order-disorder transition should be minor especially for diblocks with low conformational asymmetry [66].

Inaccuracies in the theoretical prediction for the ODT are only one source of uncertainty in the final value of $\chi$. Other sources are the uncertainties in the experimental measurement of the ODT as well as the experimental characterization of the molecules. With more accurate experiments and theory, a better quantitative agreement between theory and experiment could be achieved. This could resolve outstanding questions regarding the universality of $\chi[26,28,29]$ as well as an increase in overall precision, allowing researchers to study less prominent effects in polymer melts like chain-end effects and the finite range of interactions [67, 64]. 


\section{Bibliography}

[1] Pierre-Gilles de Gennes Scaling Concepts in Polymer Physics; CORNELL UNIVERSITY PRESS: Ithaca and London, 1979.

[2] Matsen, M. W. The Standard Gaussian Model for Block Copolymer Melts. J. Phys.: Condens. Matter 2002, 14, R21-R47.

[3] Fredrickson, G. H. The Equilibrium Theory of Inhomogeneous Polymers; Oxford University Press: New York, 2006.

[4] Matsen, M. W. In Soft Matter: Polymer Melts and Mixtures; Gompper, G., Schick, M., Eds.; Wiley-VCH: Weinheim, Germany, 2006.

[5] Schulz, G. V. The Kinetics of Chain Polymerisation V. The influence of Various Types of Reactions on the Poly-molecularity Z. Phys. Chem. (Munich) 1939, B43, 25-46.

[6] Ludwik Leibler Theory of Microphase Separation in Block Copolymers. Macromolecules 1980, 13, 1602-1617.

[7] Hong, K.M. \& Noolandi, Jaan. EFFECT OF POLYDISPERSITY ON THE MICROPHASE SEPARATION OF A BLOCK COPOLYMER SYSTEM.. Polymer communications Guildford. 1984, 25. 265-268.

[8] Listak, J.; Jakubowski, W.; Mueller, L.; Plichta, A.; Matyjaszewski, K.; Bockstaller, M. R. Effect of Symmetry of Molecular Weight Distribution in Block Copolymers on Formation of "Metastable" Morphologies. Macromolecules 2008, 41, 5919-5927.

[9] Lynd, N. A.; Hillmyer, M. A.; Matsen, M. W. Theory of Polydisperse Block Copolymer Melts: Beyond the Schulz-Zimm Distribution. Macromolecules 2008, 41, 4531-4533.

[10] David M. Cooke and An-Chang Shi Effects of Polydispersity on Phase Behavior of Diblock Copolymer Macromolecules 2006, 39, 6661-6671. 
[11] Matsen, M. W.; Bates, F. S. Unifying Weak- and Strong-Segregation Block Copolymer Theories. Macromolecules 1996, 29, 1091-1098.

[12] Ludwik Leibler Theory of Microphase Separation in Block Copolymers. Macromolecules 1980, 13, 1602-1617.

[13] Matsen, M. W. Fast and accurate SCFT calculations for periodic block-copolymer morphologies using the spectral method with Anderson mixing. Eur. Phys. J. E 2009, 30, 361-369.

[14] Glaser, J.; Qin, J.; Medapuram, P.; Müller, M.; Morse, D. C. Test of a Scaling Hypothesis for the Structure Factor of Disordered Diblock Copolymer Melts. Soft Matter 2012, 8, 11310.

[15] Fredrickson, G. H.; Helfand, E. Fluctuation Effects in the Theory of Microphase Separation in Block Copolymers. J. Chem. Phys. 1987, 87, 697-705.

[16] Kyozi Kawasaki and Takao Ohta, Phase Hamiltonian in Periodically Modulated Systems . Elsevier Science Publishers. 1986, 86, 0378-4371.

[17] Matsen, M. W. Field Theoretic Approach for Block Polymer Melts: SCFT and FTS. J. Chem. Phys. 2020, 152, 110901.

[18] Glaser, J.; Medapuram, P.; Morse, D. C. Collective and Single-Chain Correlations in Disordered Melts of Symmetric Diblock Copolymers: Quantitative Comparison of Simulations and Theory. Macromolecules 2014, 47, 851-869.

[19] Grzywacz, P.; Qin, J.; Morse, D. C. Renormalization of the One-Loop Theory of Fluctuations in Polymer Blends and Diblock Copolymer Melts. Phys. Rev. E 2007, 76, 061802 .

[20] Qin, J.; Grzywacs, P.; Morse, D. C. Renormalized One-Loop Theory of Correlations in Disordered Diblock Copolymers. J. Chem. Phys. 2011, 135, 084902.

[21] Morse, D. C.; Chung, J. K. On the Chain Length Dependence of Local Correlations in Polymer Melts and a Perturbation Theory of Symmetric Polymer Blends. J. Chem. Phys. 2009, 130, 224901.

[22] Glaser, J.; Medapuram, P.; Beardsley, T. M.; Matsen, M. W.; Morse, D. C. Universality of Block Copolymer Melts. Phys. Rev. Lett. 2014, 113, 068302. 
[23] Medapuram, P.; Glaser, J.; Morse, D. C. Universal Phenomenology of Symmetric Diblock Copolymers near the Order-Disorder Transition. Macromolecules 2015, 48, 819-839.

[24] Ghasimakbari, T.; Morse, D. C. Correlations in Disordered Melts of Asymmetric Diblock Copolymers. Macromolecules 2018, 51, 2335-2348,

[25] Russell, T. P.; Hjelm, Jr., R. P.; Seeger, P. A. Temperature Dependence of the Interaction Parameter of Polystyrene and Poly(methyl methacrylate). Macromolecules 1990, 23, 890-893.

[26] Lin, C.; Jonnalagadda, S.; Kesani, P.; Dai, H.; Balsara, N.; Effect of Molecular Structure on the Thermodynamics of Block Copolymer Melts. Macromolecules 1994, 27, $7769-7780$.

[27] Clarke, C. J.; Eisenberg, A.; La Scala, J.; Rafailovich, M. H.; Sokolov, J.; Li, Z.; Qu, S.; Nguyen, D.; Schwarz, S. A.; Strzhemechny, Y.; Sauer, B. B. Measurements of the Flory-Huggins Interaction Parameter for Polystyrene-Poly(4-vinylpyridine) Blends. Macromolecules 1997, 30, 4184-4188.

[28] Maurer, W. W.; Bates, F. S.; Lodge, T. P.; Almdal, K.; Mortensen, K.; Fredrickson, G. H. Can a Single Function for $\chi$ Account for Block Copolymer and Homopolymer Blend Phase Behavior? J. Chem. Phys. 1998, 108, 2989-3000.

[29] Miqueland-Garnier, G.; Roland, S. Beware of the Flory Parameter to Characterize Polymer-Polymer Interactions: A Critical Reexamination of the Experimental Literature. Euro. Polym. J. 2016, 84, 111-124.

[30] Arora, A.; Pillai, N.; Bates, F. S.; Dorfman, K. D. Predicting the Phase Behavior of ABAC Tetrablock Terpolymers: Sensitivity to Flory-Huggins Interaction Parameters. Polymer 2018, 154, 305-314.

[31] Ghasimakbari, T.; Morse, D. C. Order-Disorder Transitions and Free Energies in Asymmetric Diblock Copolymers. Macromolecules 2020, 53, 7399-7409.

[32] Beardsley, T. M.; Matsen, M. W. Effects of Polydispersity on the Order-Disorder Transition of Diblock Copolymer Melts. Eur. Phys. J. E 2008, 27, 323-333.

[33] M.E.J. Newman, G.T. Barkema. Monte Carlo Methods in Statistical Physics, oxford University Press 1999. 
[34] N. Metropolis, A.W. Rosenbluth, M.N Teller, E. Equation of State Calculations by Fast Computing Machines, J.Chem.Phys 21, 10871953.

[35] Beardsley, T. M.; Matsen, M. W. Monte Carlo Phase Diagram for Diblock Copolymer Melts. Eur. Phys. J. E 2010, 32, 255-264.

[36] Jian Qin, Morse, D. C. Renormalized one-loop theory of correlations in polymer blends. J. Chem. Phys. 2009, 130, 224902.

[37] Beardsley, T. M.; Matsen, M. W. Universality between Experiment and Simulation of a Diblock Copolymer Melt. Phys. Rev. Lett. 2016, 117, 217801.

[38] Willis, J. D.; Beardsley, T. M.; Matsen, M. W. Calibration of a Lattice Model for High-Molecular-Weight Block Copolymer Melts. J. Chem. Phys. 2019, 150, 204906.

[39] Cooke, D. M.; Shi, A.-C. Effects of Polydispersity on Phase Behavior of Diblock Copolymers. Macromolecules 2006, 39, 6661-6671.

[40] Matsen, M. W. Effects of Large Degrees of Polydispersity on Strongly-Segregated Block Copolymers. Eur. Phys. J. E 2006, 21, 199-207.

[41] Fetters; L. J.; Lohse, D. J.; Richter, D.; Witten, T. A.; Zirkel A. Connection between Polymer Molecular Weight, Density, Chain Dimensions, and Melt Viscoelastic Properties. Macromolecules 1994, 27, 4639-4647.

[42] Anderson, K. S.; Hillmyer, M. A. Melt Chain Dimensions of Polylactide. Macromolecules 2004, 37, 1857-1862.

[43] Henton, D. E.; Gruber, P.; Lunt, J.; Randall, J. In Natural Fibers, Biopolymers, and Biocomposites, Mohanty, A. K., Misra, M., Drzal, L. T., Eds.; CRC Press: New York, 2005.

[44] Owens, J. N.; Gancarz, I. S.; Koberstein, J. T.; Russell, T. P. Investigation of the Microphase Separation Transition in Low-Molecular-Weight Diblock Copolymers. Macromolecules 1989, 22, 3380-3387.

[45] Koch, T.; Strobl, G. R. Concentration Dependence of the Flory-Huggins Interaction Parameter of a Polymer Blend as Determined by Smal-Angle X-Ray Scattering Experiments. J. Polym. Sci., Part B 1990, 28, 343-353. 
[46] Dai, K. H.; Kramer, E. J. Determining the Temperature-Dependent Flory Interaction Parameter for Strongly Immiscible Polymers from Block Copolymer Segregation Measurements. Polymer 1994, 35, 157-161.

[47] Zha, W.; Han, C. D.; Lee, D. H.; Han, S. H.; Kim, J. K.; Kang, J. H; Park, C. Origin of the Difference in Order-Disorder Transition Temperature between Polystyreneblock-poly(2-vinylpyridine) and Polystyrene-block-poly(4-vinylpyridine) Copolymers. Macromolecules 2007, 40, 2109-2119.

[48] Han, S. H.; Kim, J. K. Temperature-Dependent Interaction Parameters of Poly(methyl methacrylate)/Poly(2-vinyl pyridine) and Poly(methyl methacrylate)/Poly(4-vinyl pyridine) Pairs. React. Funct. Polym. 2009, 69, 493-497.

[49] Sweat, D. P.; Kim, M.; Larson, S. R.; Choi, J. W.; Choo, Y.; Osuji, C. O.; Gopalan, P. Rational Design of a Block Copolymer with a High Interaction Parameter. Macromolecules 2014, 47, 6687-6696.

[50] Hofman, A. H.; Alberda van Ekenstein, G. O. R.; Woortman, A. J. J.; ten Brinke, G.; Loos, K. Poly(4-vinylpyridine)-block-Poly(N-acryloylpiperidine) Diblock Copolymers: Synthesis, Self-Assembly and Interaction. Polym. Chem. 2015, 6, 7015-7026.

[51] Zhou, S. X.; Janes, D. W.; Kim, C. B.; Willson, C. G.; Ellison, C. J. Designing Intrablock Attractions to Increase the Chi Parameter of a Symmetric Diblock Copolymer. Macromolecules 2016, 49, 8332-8340.

[52] Burns, A. B.; Christie, D.; Mulhearn, W. D.; Register, R. A. Estimating the Segregation Strength of Microphase-Separated Diblock Copolymers from the Interfacial Width. J. Polym. Sci., Part B 2019, 57, 932-940.

[53] Bates, F. S.; Schultz, M. F.; Rosedale, J. H.; Almdal, K. Correlation of Binary Polyolefin Phase Behavior with Statistical Segment Length Asymmetry. Macromolecules 1992, 25, 5547-5550.

[54] Cochran, E. W.; Bates, F. S. Thermodynamic Behavior of Poly(cyclohexylethylene) in Polyolefin Diblock Copolymers. Macromolecules 2002, 35, 7368-7374.

[55] Almdal K.; Hillmyer, M. A.; Bates, F. S. Influence of Conformational Asymmetry on Polymer-Polymer Interactions: An Entropic or Enthalpic Effect? Macromolecules 2002, 35, 7685-7691. 
[56] Schmidt, S. C.; Hillmyer, M. A. Morphological Behavior of Model Poly(ethylenealt-propylene)-b-Polylactide Diblock Copolymers. J. Polym. Sci., Part B 2002, 40, $2364-2376$.

[57] Zalusky, A. S.; Olayo-Valles, R.; Wolf, J. H.; Hillmyer, M. A. Ordered Nanoporous Polymers from Polystyrene-Polylactide Block Copolymers. J. Am. Chem. Soc. 2002, 124, 12761-12773.

[58] Lee, S.; Gillard, T. M.; Bates, F. S. Fluctuations, Order, and Disorder in Short Diblock Copolymers. AIChE J. 2013, 59, 3502-3513.

[59] Kennemur, J. G.; Yao, L.; Bates, F. S.; Hillmyer, M. A. Sub-5 nm Domains in Ordered Poly(cyclohexylethylene)-block-Poly(methyl methacrylate) Block Polymers for Lithography. Macromolecules 2014, 47, 1411-1418.

[60] Schulze, M. W.; Lewis III, R. M.; Lettow, J. H.; Hickey, R. J.; Gillard, T. M.; Hillmyer, M. A.; Bates, F. S. Conformational Asymmetry and Quasicrystal Approximants in Linear Diblock Copolymers. Phys. Rev. Lett. 2017, 118, 207801.

[61] Rosedale, J. H.; Bates, F. S.; Almdal, K.; Mortensen, K.; Wignall, G. D. Order and Disorder in Symmetric Diblock Copolymer Melts. Macromolecules 1995, 28, 14291443.

[62] Zimm, B. H. Apparatus and Methods for Measurement and Interpretation of the Angular Variation of Light Scattering; Preliminary Results on Polystyrene Solutions. J. Chem. Phys. 1948, 16, 1099-1116.

[63] Arora, A.; Morse, D. C.; Bates, F. S.; Dorfman, K. D. Commensurability and Finite Size Effects in Lattice Simulations of Diblock Copolymers. Soft Matter 2015, 11, 4862-4867.

[64] Frielinghaus, H.; Hermsdorf, N.; Sigel, R.; Almdal, K.; Mortensen, K.; Hamley, I. W .; Messe, L.; Corvazier, L.; Ryan, A. J.; van Dusschoten, D.; Wilhelm, M.; Foudas, G.; Fytas, G. Blends of AB/BC Diblock Copolymers with a Large Interaction Parameter

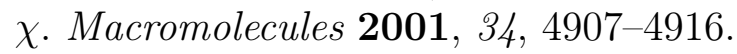

[65] Arora, A.; Pillai, N.; Bates, F. S.; Dorfman, K. D. Erratum to "Predicting the Phase Behavior of ABAC Tetrablock Terpolymers: Sensitivity to Flory-Huggins Interaction Parameters" [Polymer, 154 (2018), 305-314]. Polymer 2020, 210, 123038. 
[66] Matsen, M. W.; Bates, F. S. Conformationally Asymmetric Diblock Copolymers. J. Polym. Sci., Part B 1997, 35, 945-952.

[67] Kim, J.; Jung, H. Y.; Park, M. J. End-Group Chemistry and Junction Chemistry in Polymer Science: Past, Present, and Future. Macromolecules (2020), 53, 746-763. 
Appendices 


\section{Appendix A}

\section{Calibration Data}

The method we use for calibration of the 19 chemical pairs from Chapter 5 is presented here in more detail. we start by finding the value of $\bar{N}=\rho_{0}^{2} a^{6} N$ associated with each of the diblock copolymer melts from the experimental data. The next step is to find the statistical segment length $a$. The mass density of the A-block, $\rho_{A}$, is multiplied by a standard segment volume, $\rho_{0}^{-1}=118^{3}$, to give the segment mass of the polymer's A side, $\rho_{A} / \rho_{0}$. The total number-averaged mass of the A-block, $M_{n, A}$, is then divided by the mass of a segment to give the number of segments in the chain,

$$
N_{A}=\frac{M_{n, A} \rho_{0}}{\rho_{A}} .
$$

A similar process is also done for the $B$ side of the diblock to give

$$
N_{B}=\frac{M_{n, B} \rho_{0}}{\rho_{B}} .
$$

This gives the total chain length as $N=N_{A}+N_{B}$. An equation that combines these results is,

$$
N=\frac{M_{N} \rho_{0}}{N_{A v}\left(f \rho_{A}+(1-f) \rho_{B}\right)},
$$

where the factor $N_{A v}$, Avogadro's number, exists to convert between units. Next, the statistical segment length is found using its relation to the average end-to-end length squared $R_{0}^{2}=a^{2} N$, as given in Equation, 1.4. To find thestatistical segment length of the A-block, $a_{A}$, we can rearrange Equation 1.4 to isolate for $a$, then substitute data from the literature for $\left(R_{0}^{2} / M_{n, A}\right)$ [41]. This results in the statistical segment lengths

$$
a_{A}^{2}=N_{A}\left(R^{2} / M_{n}\right)_{A}
$$


and

$$
a_{B}^{2}=N_{B}\left(R^{2} / M_{n}\right)_{B},
$$

for the A and B blocks, respectively. Tabulations of the statistical segment length for various chemistries are shown below.

\begin{tabular}{cccc} 
Chemistry & $R_{0}^{2} / M_{N}$ & $\rho$ & $a$ \\
\hline \hline PE[41] & 1.250 & 0.784 & 8.35 \\
PEE[41] & 0.507 & 0.807 & 5.39 \\
PEP[41] & 0.834 & 0.790 & 6.84 \\
PCHE[41] & 0.323 & 0.920 & 4.60 \\
PI[41] & 0.625 & 0.830 & 6.07 \\
PS[41] & 0.434 & 0.969 & 5.47 \\
PEO[41] & 0.805 & 1.064 & 7.80 \\
PMMA[41] & 0.425 & 1.130 & 5.84 \\
PLA[42, 43] & 0.605 & 1.152 & 7.03 \\
PDMS[41] & 0.457 & 0.895 & 5.39 \\
\hline
\end{tabular}

Table A.1: Molecular data at $T=140^{\circ} \mathrm{C}$ used in calculating the number of segments and the segment mass. $R_{0}^{2} / M_{N}$ is how the radius of gyration scales with chain length from the literature. $\rho$ is the density from the literature. $a$ is the calculated statistical segment length.

Using the data in Table A.1, $\bar{N}$ can be calculated independently for each of the melts used in the calibration,

$$
\bar{N}=\rho_{0}^{2}\left(f a_{A}^{2}+(1-f) a_{B}^{2}\right)^{3} N .
$$

These calibrations for $\chi$ are performed by calculating the value of $(\chi N)_{O D T}$ for each of the melts used in the calibration. With the four different theoretical predictions,

$$
\begin{aligned}
& (\chi N)_{\mathrm{ODT}}^{M F}=10.495, \\
& (\chi N)_{\mathrm{ODT}}^{F H}=10.495+41.0 \bar{N}^{-1 / 3}, \\
& (\chi N)_{\mathrm{ODT}}^{U n i}=10.495+41.0 \bar{N}^{-1 / 3}+123.0 \bar{N}^{-0.56}, \\
& (\chi N)_{\mathrm{ODT}}=(\chi N)_{\mathrm{ODT}}^{U n i}\left(1+\kappa(\mathrm{\Xi}-1)+\gamma\left(f-\frac{1}{2}\right)^{2}\right),
\end{aligned}
$$

the experimental ODT temperature, $T_{O D T}$, of the melts it plotted on the x-axis and the theoretical $\chi$ is plotted on the y-axis. The relation between $\chi$ and $T$ is determined by fitting to $\chi(T)=A / T+B$. These fits are shown in the following figures. 


\section{Poly(ethylene)-Poly(ethylenepropylene)}

\begin{tabular}{cccc}
\multicolumn{4}{c}{ PE-PEP diblock polymers } \\
\hline $\begin{array}{c}M_{n} \\
(\mathrm{~kg} / \mathrm{mol})\end{array}$ & $f_{\mathrm{PE}}$ & $Đ$ & $\begin{array}{c}T_{\text {ODT }} \\
\left({ }^{\circ} \mathrm{C}\right)\end{array}$ \\
\hline 88 & 0.50 & 1.06 & 119 \\
107 & 0.49 & 1.07 & 139 \\
117 & 0.50 & 1.09 & 139 \\
128 & 0.49 & 1.06 & 159 \\
\hline
\end{tabular}

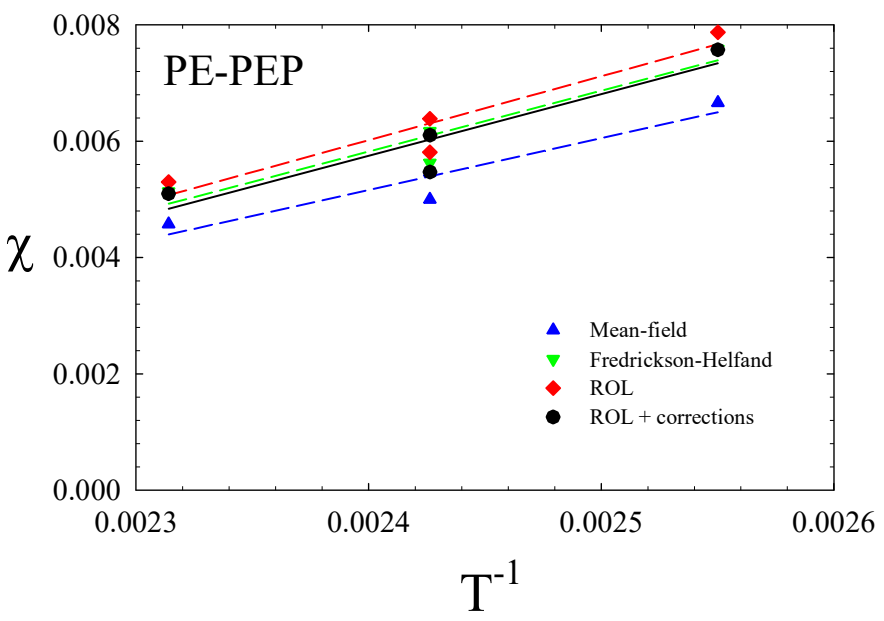

Fits to $\chi(T)=A / T+B$ for PE-PEP

\begin{tabular}{|c|c|c|}
\hline $\begin{array}{l}\text { Prediction } \\
(\chi N)_{\mathrm{ODT}}\end{array}$ & $A$ & $B \times 10^{3}$ \\
\hline Mean-field & 8.91 & -16.21 \\
\hline Fredrickson-Helfand & 10.45 & -19.26 \\
\hline $\mathrm{ROL}$ & 10.99 & -20.36 \\
\hline ROL + corrections & 10.60 & -19.68 \\
\hline
\end{tabular}




\section{Poly(ethylenepropylene)-Poly(ethylethylene)}

PEP-PEE diblock polymers

\begin{tabular}{cccc}
\hline $\begin{array}{c}M_{n} \\
(\mathrm{~kg} / \mathrm{mol})\end{array}$ & $f_{\text {PEP }}$ & $Đ$ & $\begin{array}{c}T_{\text {ODT }} \\
\left({ }^{\circ} \mathrm{C}\right)\end{array}$ \\
\hline 44.3 & 0.54 & 1.08 & 16 \\
50.1 & 0.56 & 1.07 & 96 \\
54.8 & 0.55 & 1.05 & 125 \\
81.2 & 0.53 & 1.05 & 291 \\
\hline
\end{tabular}

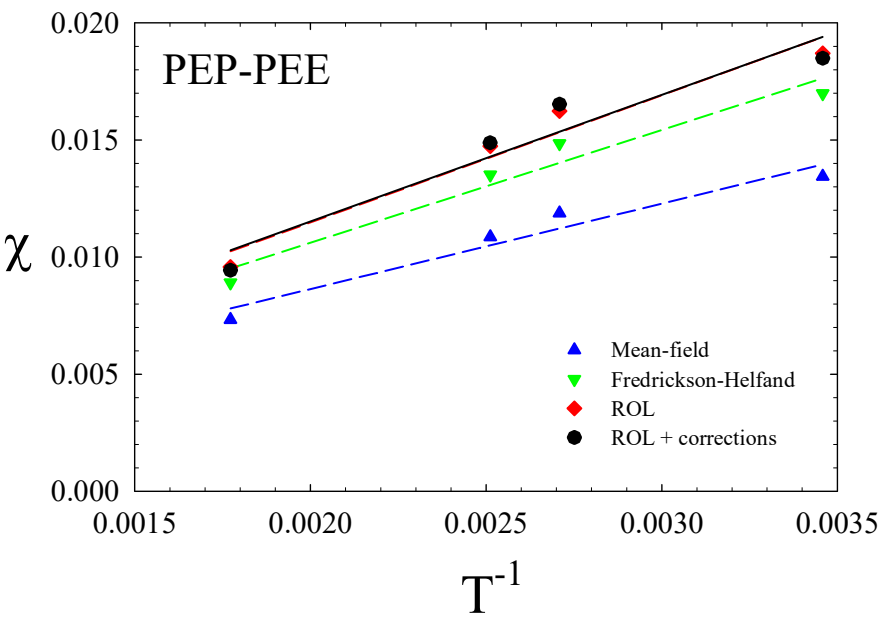

Fits to $\chi(T)=A / T+B$ for PEP-PEE

\begin{tabular}{lcc}
\hline $\begin{array}{l}\text { Prediction for } \\
(\chi N)_{\text {ODT }}\end{array}$ & $A$ & $B \times 10^{3}$ \\
\hline Mean-field & 3.65 & 1.35 \\
Fredrickson-Helfand & 4.81 & 0.99 \\
ROL & 5.43 & 0.62 \\
ROL + corrections & 5.40 & 0.72 \\
\hline
\end{tabular}




\section{Poly(cyclohexylethylene)-Poly(ethylethylene)}

PCHE-PEE diblock polymers

\begin{tabular}{cccc}
\hline $\begin{array}{c}M_{n} \\
(\mathrm{~kg} / \mathrm{mol})\end{array}$ & $f_{\mathrm{PCHE}}$ & $Đ$ & $\begin{array}{c}T_{\mathrm{ODT}} \\
\left({ }^{\circ} \mathrm{C}\right)\end{array}$ \\
\hline 37.9 & 0.48 & 1.02 & 184 \\
39.0 & 0.50 & 1.03 & 177 \\
40.6 & 0.50 & 1.02 & 180 \\
46.9 & 0.51 & 1.03 & 236 \\
53.1 & 0.50 & 1.02 & 273 \\
62.3 & 0.50 & 1.02 & 335 \\
\hline
\end{tabular}

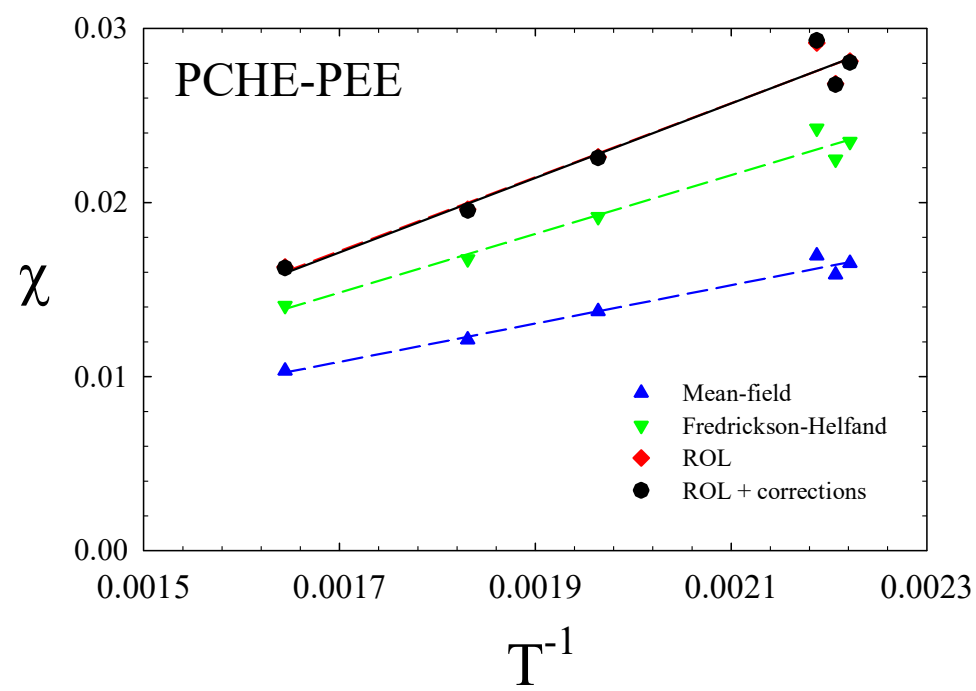

Fits to $\chi(T)=A / T+B$ for PCHE-PEE

\begin{tabular}{|c|c|c|}
\hline $\begin{array}{l}\text { Prediction } \\
(\chi N)_{\mathrm{ODT}}\end{array}$ & $A$ & $B \times 10^{3}$ \\
\hline Mean-field & 11.0 & -7.8 \\
\hline Fredrickson-Helfand & 16.9 & -13.8 \\
\hline ROL & 21.4 & -18.9 \\
\hline ROL + corrections & 21.6 & -19.2 \\
\hline
\end{tabular}




\section{Poly(ethylene)-Poly(ethylethylene)}

PE-PEE diblock polymers

\begin{tabular}{cccc}
\hline $\begin{array}{c}M_{n} \\
(\mathrm{~kg} / \mathrm{mol})\end{array}$ & $f_{\mathrm{PE}}$ & $Đ$ & $\begin{array}{c}T_{\mathrm{ODT}} \\
\left({ }^{\circ} \mathrm{C}\right)\end{array}$ \\
\hline 23.9 & 0.50 & 1.15 & 136 \\
27.2 & 0.48 & 1.05 & 182 \\
\hline
\end{tabular}

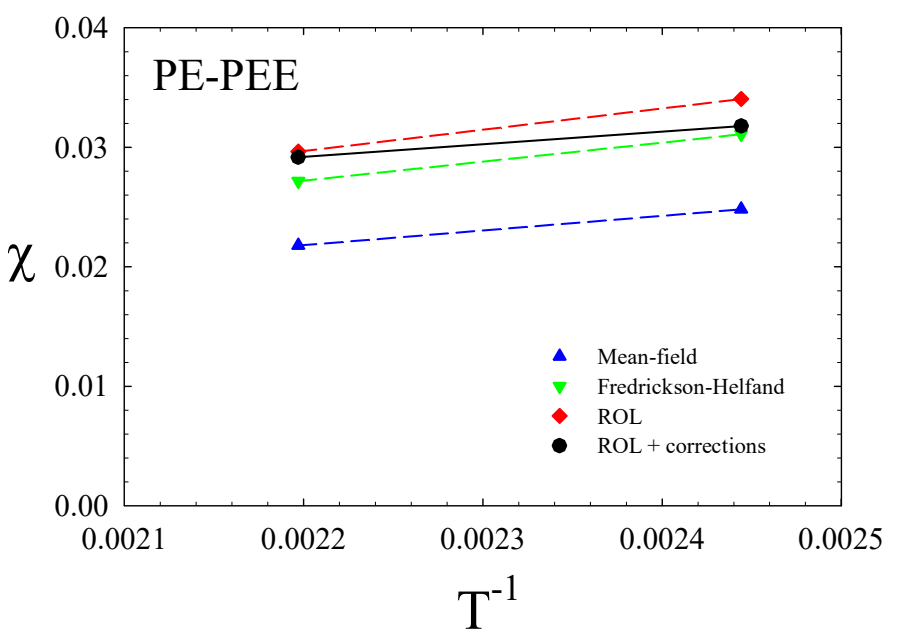

Fits to $\chi(T)=A / T+B$ for PE-PEE

\begin{tabular}{lcc}
\hline $\begin{array}{l}\text { Prediction for } \\
(\chi N)_{\text {ODT }}\end{array}$ & $A$ & $B \times 10^{3}$ \\
\hline Mean-field & 12.2 & -5.0 \\
Fredrickson-Helfand & 15.9 & -7.8 \\
ROL & 17.8 & -9.5 \\
ROL + corrections & 10.5 & 6.0 \\
\hline
\end{tabular}




\section{Poly(cyclohexylethylene)-Poly(ethylenepropylene)}

\begin{tabular}{cccc}
\multicolumn{4}{c}{ PCHE-PEP diblock polymers } \\
\hline $\begin{array}{c}M_{n} \\
(\mathrm{~kg} / \mathrm{mol})\end{array}$ & $f_{\text {PCHE }}$ & $Đ$ & $\begin{array}{c}T_{\text {ODT }} \\
\left({ }^{\circ} \mathrm{C}\right)\end{array}$ \\
\hline 18.1 & 0.52 & 1.02 & 139 \\
20.1 & 0.50 & 1.02 & 175 \\
21.4 & 0.51 & 1.02 & 190 \\
21.4 & 0.55 & 1.02 & 192 \\
22.0 & 0.48 & 1.02 & 208 \\
22.0 & 0.51 & 1.02 & 201 \\
23.4 & 0.51 & 1.02 & 243 \\
24.6 & 0.52 & 1.02 & 253 \\
\hline
\end{tabular}

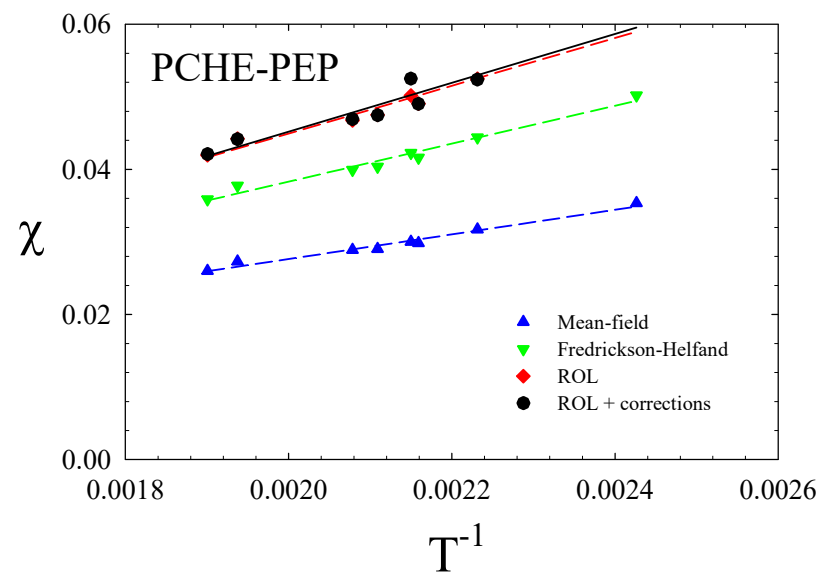

Fits to $\chi(T)=A / T+B$ for PCHE-PEP

\begin{tabular}{|c|c|c|}
\hline $\begin{array}{l}\text { Prediction } \\
(\chi N)_{\mathrm{ODT}}\end{array}$ & $A$ & $B \times 10^{3}$ \\
\hline Mean-field & 17.0 & -6.3 \\
\hline Fredrickson-Helfand & 26.1 & -14.0 \\
\hline $\mathrm{ROL}$ & 33.0 & -21.1 \\
\hline ROL + corrections & 33.7 & -22.2 \\
\hline
\end{tabular}




\section{Poly(ethylethylene)-Poly(dimethylsiloxane)}

PEE-PDMS diblock polymers

\begin{tabular}{cccc}
\hline $\begin{array}{c}M_{n} \\
(\mathrm{~kg} / \mathrm{mol})\end{array}$ & $f_{\mathrm{PEE}}$ & $Đ$ & $\begin{array}{c}T_{\mathrm{ODT}} \\
\left({ }^{\circ} \mathrm{C}\right)\end{array}$ \\
\hline 10.4 & 0.48 & 1.07 & 110 \\
15.3 & 0.50 & 1.07 & 165 \\
\hline
\end{tabular}

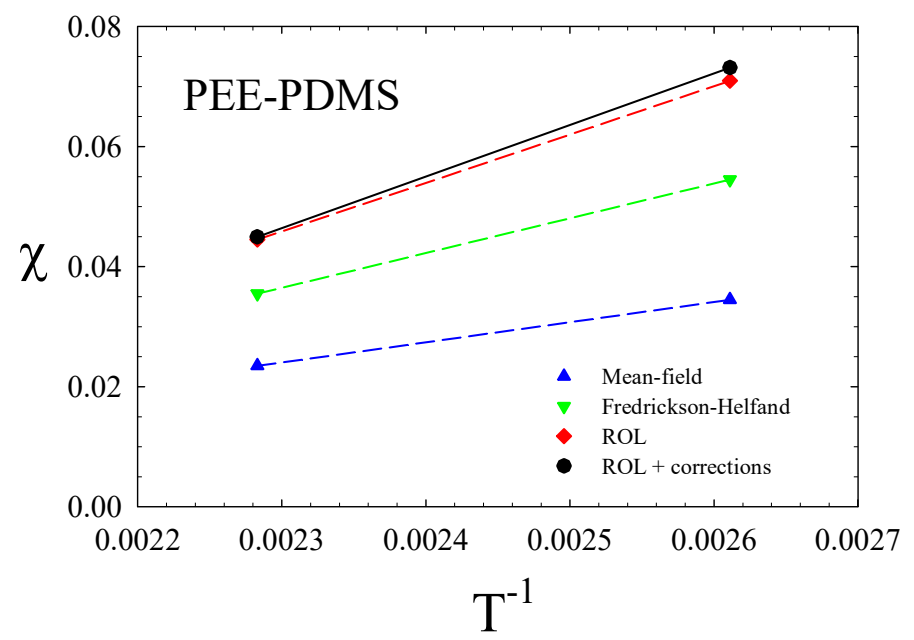

Fits to $\chi(T)=A / T+B$ for PEE-PDMS

\begin{tabular}{lcc}
\hline $\begin{array}{l}\text { Prediction for } \\
(\chi N)_{\text {ODT }}\end{array}$ & $A$ & $B \times 10^{3}$ \\
\hline Mean-field & 33.5 & -53.1 \\
Fredrickson-Helfand & 58.0 & -97.0 \\
ROL & 80.3 & -139.6 \\
ROL + corrections & 86.0 & -151.3 \\
\hline
\end{tabular}




\section{Poly(cyclohexylethylene)-Poly(ethylene)}

PCHE-PE diblock polymers

\begin{tabular}{cccc}
\hline $\begin{array}{c}M_{n} \\
(\mathrm{~kg} / \mathrm{mol})\end{array}$ & $f_{\text {PCHE }}$ & $Đ$ & $\begin{array}{c}T_{\text {ODT }} \\
\left({ }^{\circ} \mathrm{C}\right)\end{array}$ \\
\hline 12.8 & 0.54 & 1.02 & 168 \\
13.2 & 0.55 & 1.02 & 172 \\
13.6 & 0.54 & 1.02 & 180 \\
15.6 & 0.52 & 1.02 & 227 \\
16.2 & 0.53 & 1.02 & 234 \\
16.4 & 0.52 & 1.02 & 250 \\
16.9 & 0.53 & 1.03 & 263 \\
17.0 & 0.52 & 1.03 & 266 \\
\hline
\end{tabular}

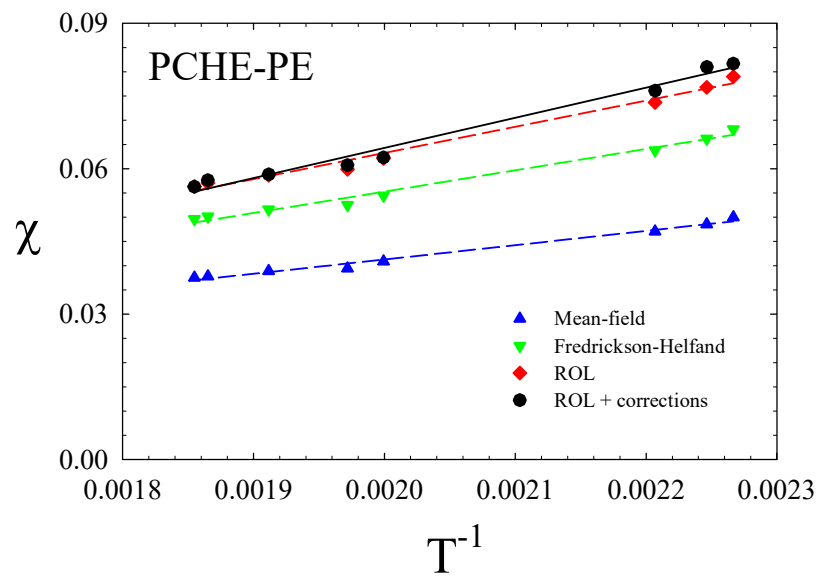

Fits to $\chi(T)=A / T+B$ for PCHE-PE

\begin{tabular}{|c|c|c|}
\hline $\begin{array}{l}\text { Prediction } \\
(\chi N)_{\text {ODT }}\end{array}$ & $A$ & $B \times 10^{3}$ \\
\hline Mean-field & 29.5 & -17.7 \\
\hline Fredrickson-Helfand & 44.0 & -32.7 \\
\hline ROL & 54.1 & -44.9 \\
\hline ROL + corrections & 62.1 & -60.0 \\
\hline
\end{tabular}




\section{Poly(styrene)-Poly(isoprene)}

\begin{tabular}{cccc}
\multicolumn{4}{c}{ PS-PI diblock polymers } \\
\hline $\begin{array}{c}M_{n} \\
(\mathrm{~kg} / \mathrm{mol})\end{array}$ & $f_{\mathrm{PS}}$ & $Đ$ & $\begin{array}{c}T_{\mathrm{ODT}} \\
\left({ }^{\circ} \mathrm{C}\right)\end{array}$ \\
\hline 12.4 & 0.46 & 1.05 & 78 \\
13.7 & 0.46 & 1.05 & 84 \\
20.1 & 0.54 & 1.03 & 117 \\
27.8 & 0.56 & 1.04 & 200 \\
\hline
\end{tabular}

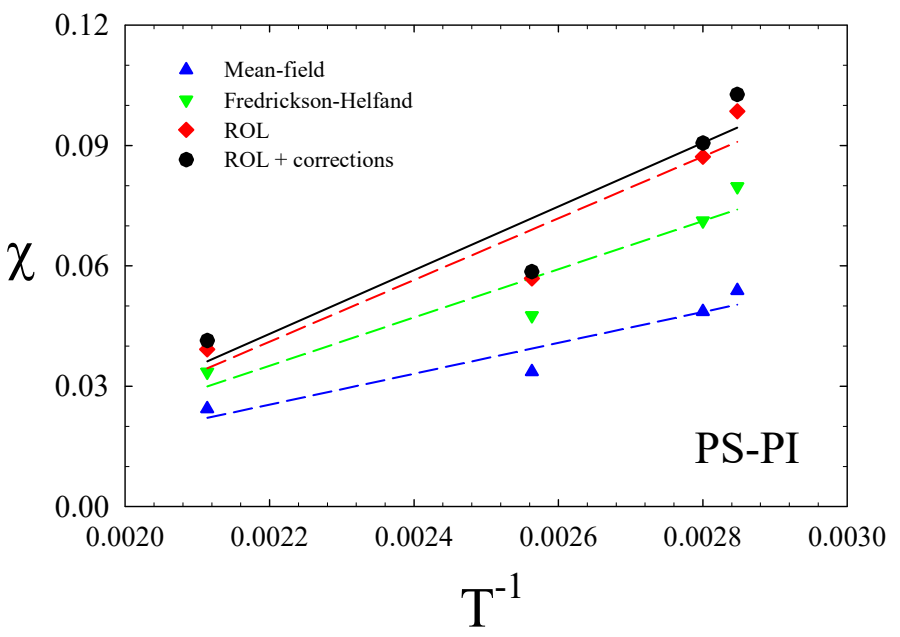

Fits to $\chi(T)=A / T+B$ for PS-PI

\begin{tabular}{|c|c|c|}
\hline $\begin{array}{l}\text { Prediction } \\
(\chi N)_{\mathrm{ODT}}\end{array}$ & $A$ & $B \times 10^{3}$ \\
\hline Mean-field & 38.5 & -59.2 \\
\hline Fredrickson-Helfand & 60.1 & -97.1 \\
\hline ROL & 77.0 & -128.2 \\
\hline ROL + corrections & 79.3 & -131.5 \\
\hline
\end{tabular}




\section{Poly(DL-lactide)-Poly(styrene)}

PLA-PS diblock polymers

\begin{tabular}{cccc}
\hline $\begin{array}{c}M_{n} \\
(\mathrm{~kg} / \mathrm{mol})\end{array}$ & $f_{\text {PLA }}$ & $Đ$ & $\begin{array}{c}T_{\text {ODT }} \\
\left({ }^{\circ} \mathrm{C}\right)\end{array}$ \\
\hline 9.5 & 0.45 & 1.11 & 116 \\
12 & 0.54 & 1.15 & 164 \\
15 & 0.43 & 1.07 & 213 \\
15 & 0.44 & 1.12 & 216 \\
18 & 0.52 & 1.09 & 268 \\
19 & 0.54 & 1.22 & 258 \\
\hline
\end{tabular}

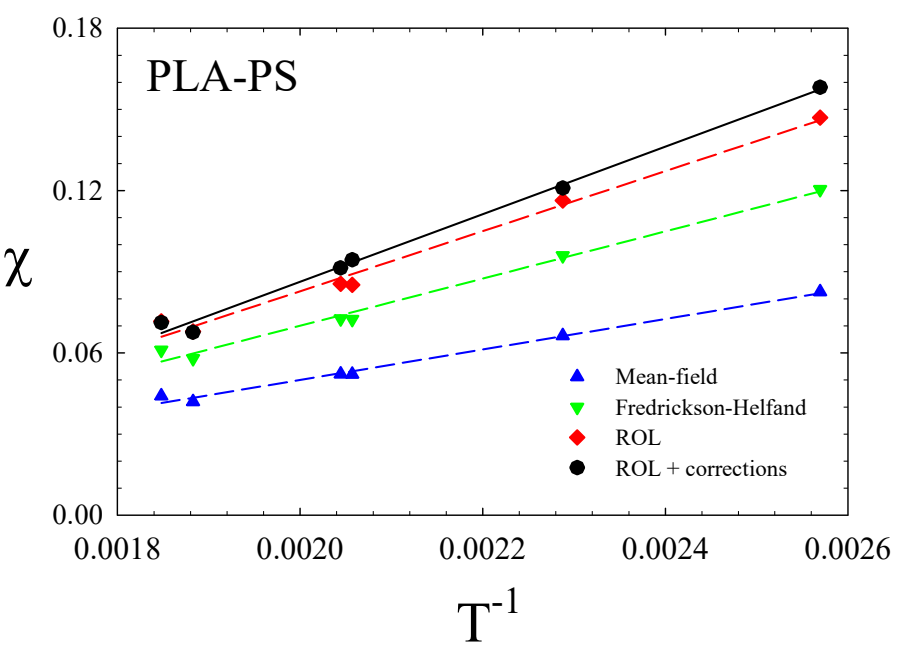

Fits to $\chi(T)=A / T+B$ for PLA-PS

\begin{tabular}{lcc}
\hline $\begin{array}{l}\text { Prediction } \\
(\chi N)_{\text {ODT }}\end{array}$ & $A$ & $B \times 10^{3}$ \\
\hline Mean-field & 56 & -62 \\
Fredrickson-Helfand & 87 & -104 \\
ROL & 111 & -139 \\
ROL + corrections & 125 & -163 \\
\hline
\end{tabular}




\section{Poly(ethylene)-Poly(dimethylsiloxane)}

PE-PDMS diblock polymers

\begin{tabular}{cccc}
\hline $\begin{array}{c}M_{n} \\
\mathrm{~kg} / \mathrm{mol})\end{array}$ & $f_{\mathrm{PE}}$ & $Đ$ & $\begin{array}{c}T_{\mathrm{ODT}} \\
\left({ }^{\circ} \mathrm{C}\right)\end{array}$ \\
\hline 6.8 & 0.49 & 1.09 & 225 \\
6.0 & 0.50 & 1.14 & 185 \\
\hline
\end{tabular}

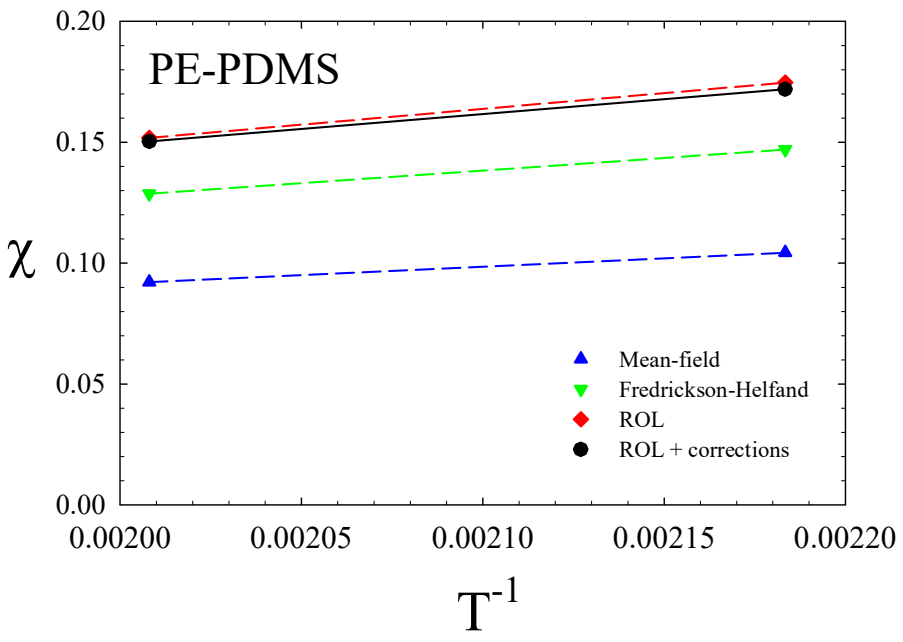

Fits to $\chi(T)=A / T+B$ for PE-PDMS

\begin{tabular}{|c|c|c|}
\hline $\begin{array}{l}\text { Prediction } \\
(\chi N)_{\mathrm{ODT}}\end{array}$ & $A$ & $B \times 10^{3}$ \\
\hline Mean-field & 69 & -47 \\
\hline Fredrickson-Helfand & 105 & -81 \\
\hline ROL & 131 & -110 \\
\hline ROL + corrections & 123 & -97 \\
\hline
\end{tabular}




\section{Poly(ethylenepropylene)-Poly(dimethylsiloxane)}

PEP-PDMS diblock polymers

\begin{tabular}{cccc}
\hline $\begin{array}{c}M_{n} \\
(\mathrm{~kg} / \mathrm{mol})\end{array}$ & $f_{\mathrm{PEP}}$ & $Đ$ & $\begin{array}{c}T_{\mathrm{ODT}} \\
\left({ }^{\circ} \mathrm{C}\right)\end{array}$ \\
\hline 6.3 & 0.48 & 1.07 & 64 \\
7.7 & 0.50 & 1.13 & 165 \\
\hline
\end{tabular}

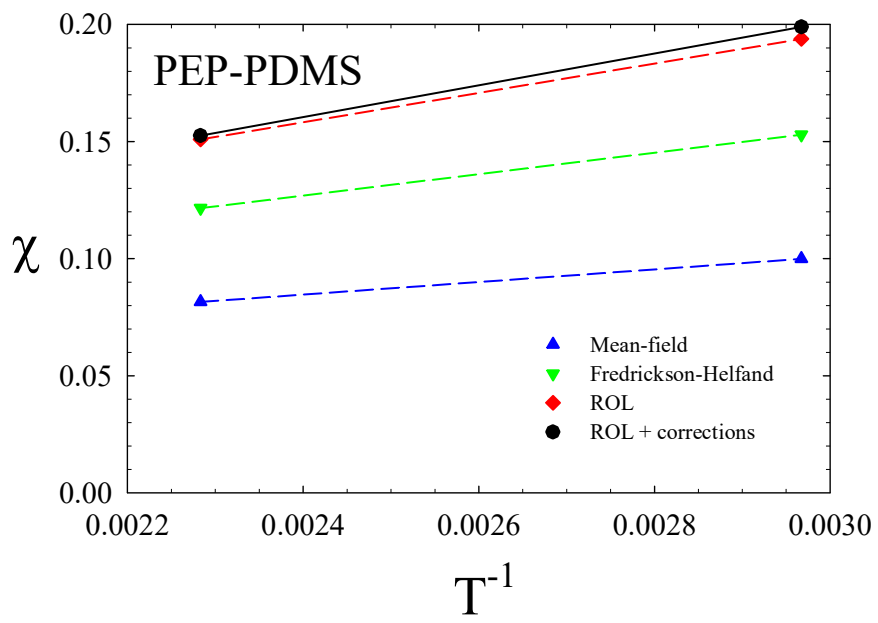

Fits to $\chi(T)=A / T+B$ for PEP-PDMS

\begin{tabular}{lcc}
\hline $\begin{array}{l}\text { Prediction for } \\
(\chi N)_{\text {ODT }}\end{array}$ & $A$ & $B \times 10^{3}$ \\
\hline Mean-field & 27 & -20 \\
Fredrickson-Helfand & 46 & -17 \\
ROL & 63 & -8 \\
ROL + corrections & 68 & -2 \\
\hline
\end{tabular}




\section{Poly(isoprene)-Poly(ethyleneoxide)}

PI-PEO diblock polymers

\begin{tabular}{cccc}
\hline $\begin{array}{c}M_{n} \\
(\mathrm{~kg} / \mathrm{mol})\end{array}$ & $f_{\mathrm{PI}}$ & $Đ$ & $\begin{array}{c}T_{\mathrm{ODT}} \\
\left({ }^{\circ} \mathrm{C}\right)\end{array}$ \\
\hline 4.15 & 0.5 & 1.06 & 132 \\
6.73 & 0.49 & 1.10 & 247 \\
\hline
\end{tabular}

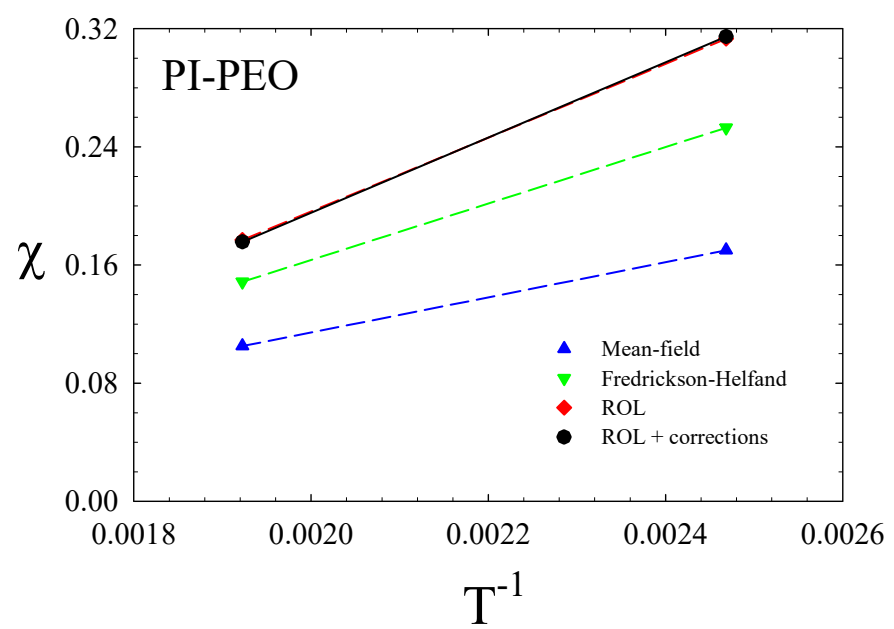

Fits for PI-PEO $\chi$ parameter

\begin{tabular}{lll}
\hline & $A$ & $B \times 10^{3}$ \\
\hline Mean-field & 119 & -123 \\
Fredrickson- & 191 & -219 \\
Helfand & & \\
ROL & 250 & -303 \\
ROL + corrections & 255 & -314 \\
\hline
\end{tabular}




\section{Poly(cyclohexylethylene)-Poly(methylmethacrylate)}

\begin{tabular}{cccc}
\multicolumn{4}{c}{ PCHE-PMMA diblock polymers } \\
\hline $\begin{array}{c}M_{n} \\
(\mathrm{~kg} / \mathrm{mol})\end{array}$ & $f_{\mathrm{PCHE}}$ & $Đ$ & $\begin{array}{c}T_{\mathrm{ODT}} \\
\left({ }^{\circ} \mathrm{C}\right)\end{array}$ \\
\hline 10.1 & 0.45 & 1.10 & 332 \\
9.3 & 0.48 & 1.11 & 325 \\
5.6 & 0.45 & 1.12 & 204 \\
4.8 & 0.46 & 1.11 & 173 \\
\hline
\end{tabular}

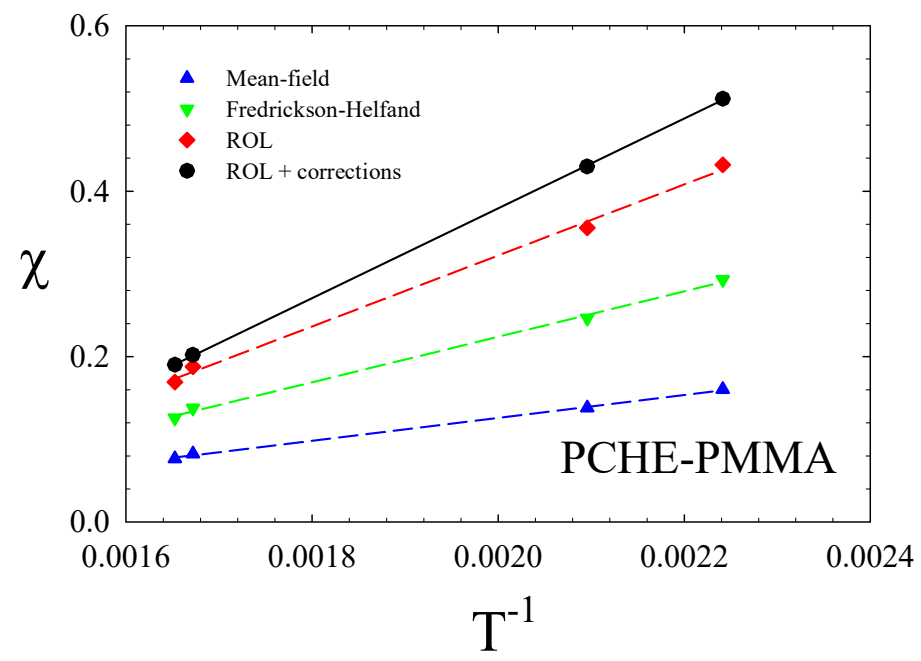

Fits to $\chi(T)=A / T+B$ for PCHE-PMMA

\begin{tabular}{|c|c|c|}
\hline $\begin{array}{l}\text { Prediction } \\
(\chi N)_{\text {ODT }}\end{array}$ & $A$ & $B \times 10^{3}$ \\
\hline Mean-field & 138 & -151 \\
\hline Fredrickson-Helfand & 275 & -325 \\
\hline ROL & 429 & -536 \\
\hline ROL + corrections & 543 & -707 \\
\hline
\end{tabular}




\section{Poly(DL-lactide)-Poly(ethylethylene)}

PLA-PEE diblock polymers

\begin{tabular}{cccc}
\hline $\begin{array}{c}M_{n} \\
(\mathrm{~kg} / \mathrm{mol})\end{array}$ & $f_{\mathrm{PLA}}$ & $\mathrm{Ð}$ & $\begin{array}{c}T_{\mathrm{ODT}} \\
\left({ }^{\circ} \mathrm{C}\right)\end{array}$ \\
\hline 3.3 & 0.52 & 1.06 & 165 \\
3.4 & 0.53 & 1.06 & 169 \\
3.8 & 0.48 & 1.05 & 195 \\
\hline
\end{tabular}

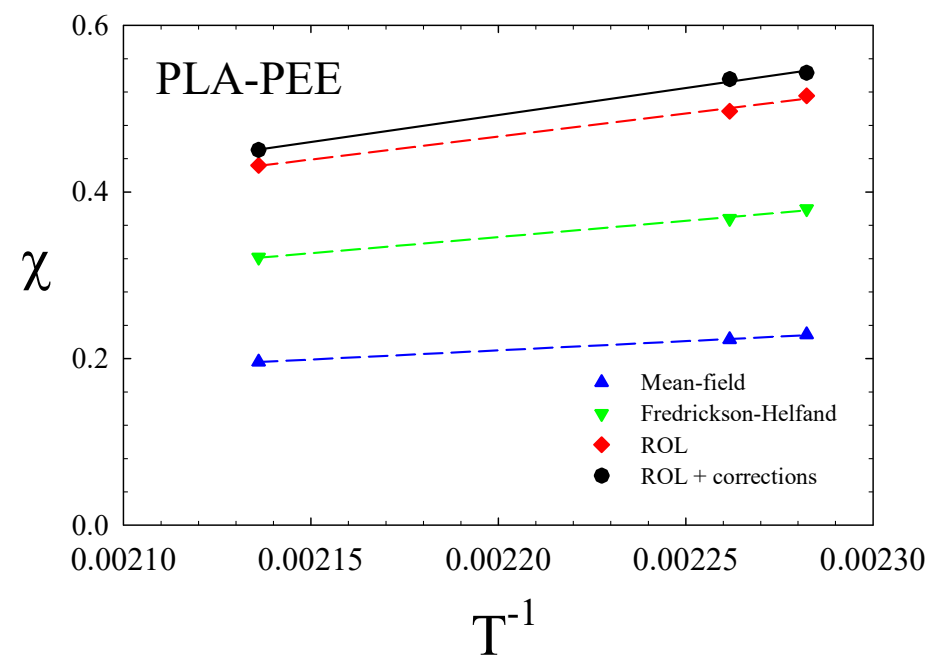

Fits to $\chi(T)=A / T+B$ for PLA-PEE

\begin{tabular}{|c|c|c|}
\hline $\begin{array}{l}\text { Prediction } \\
(\chi N)_{\mathrm{ODT}}\end{array}$ & $A$ & $B \times 10^{3}$ \\
\hline Mean-field & 220 & -275 \\
\hline Fredrickson-Helfand & 389 & -510 \\
\hline ROL & 553 & -751 \\
\hline ROL + corrections & 647 & -931 \\
\hline
\end{tabular}




\section{Poly(ethylene)-Poly(ethyleneoxide)}

PE-PEO diblock polymers

\begin{tabular}{cccc}
\hline $\begin{array}{c}M_{n} \\
(\mathrm{~kg} / \mathrm{mol})\end{array}$ & $f_{\mathrm{PE}}$ & $Đ$ & $\begin{array}{c}T_{\mathrm{ODT}} \\
\left({ }^{\circ} \mathrm{C}\right)\end{array}$ \\
\hline 2.1 & 0.48 & 1.10 & 112 \\
2.8 & 0.48 & 1.15 & 153 \\
\hline
\end{tabular}

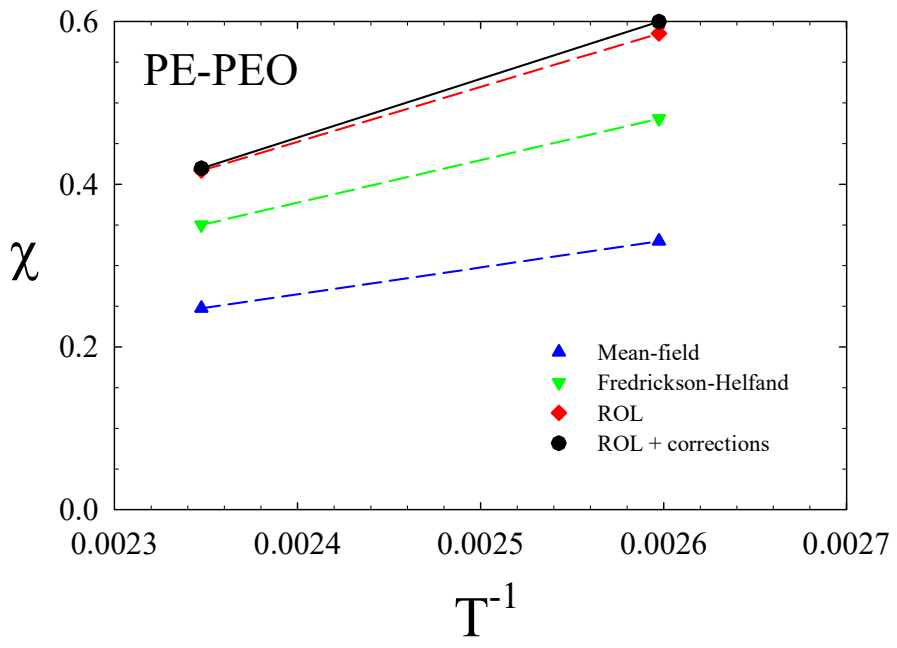

Fits to $\chi(T)=A / T+B$ for PE-PEO

\begin{tabular}{lcc}
\hline $\begin{array}{l}\text { Prediction for } \\
(\chi N)_{\text {ODT }}\end{array}$ & $A$ & $B \times 10^{3}$ \\
\hline Mean-field & 330 & -527 \\
Fredrickson-Helfand & 522 & -875 \\
ROL & 674 & -1164 \\
ROL + corrections & 722 & -1275 \\
\hline
\end{tabular}




\section{Poly(DL-lactide)-Poly(ethylenepropylene)}

PLA-PEP diblock polymers

\begin{tabular}{cccc}
\hline $\begin{array}{c}M_{n} \\
(\mathrm{~kg} / \mathrm{mol})\end{array}$ & $f_{\text {PLA }}$ & $Đ$ & $\begin{array}{c}T_{\mathrm{ODT}} \\
\left({ }^{\circ} \mathrm{C}\right)\end{array}$ \\
\hline 2.58 & 0.50 & 1.17 & 159 \\
3.28 & 0.58 & 1.15 & 170 \\
4.05 & 0.50 & 1.08 & 226 \\
4.55 & 0.55 & 1.08 & 236 \\
4.95 & 0.57 & 1.1 & 238 \\
\hline
\end{tabular}

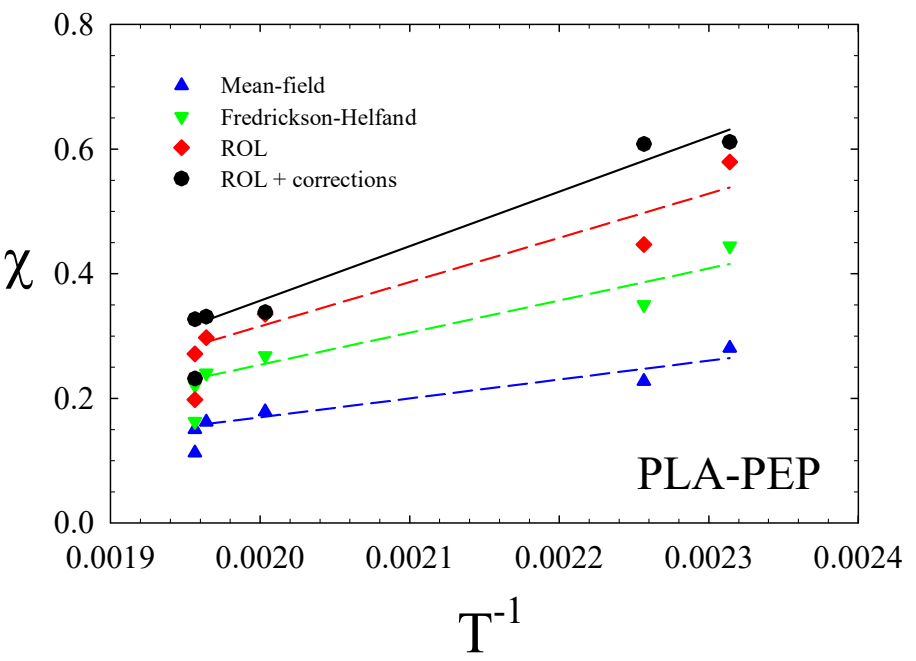

Fits to $\chi(T)=A / T+B$ for PLA-PEP

\begin{tabular}{lcc}
\hline $\begin{array}{l}\text { Prediction for } \\
(\chi N)_{\text {ODT }}\end{array}$ & $A$ & $B \times 10^{3}$ \\
\hline Mean-field & 302 & -435 \\
Fredrickson-Helfand & 516 & -778 \\
ROL & 709 & -1103 \\
ROL + corrections & 875 & -1393 \\
\hline
\end{tabular}




\section{Poly(DL-lactide)-Poly(isoprene)}

PLA-PI diblock polymers

\begin{tabular}{cccc}
\hline $\begin{array}{c}M_{n} \\
(\mathrm{~kg} / \mathrm{mol})\end{array}$ & $f_{\text {PLA }}$ & $Đ$ & $\begin{array}{c}T_{\mathrm{ODT}} \\
\left({ }^{\circ} \mathrm{C}\right)\end{array}$ \\
\hline 2.75 & 0.51 & 1.12 & 93 \\
3.55 & 0.54 & 1.09 & 110 \\
4.53 & 0.54 & 1.09 & 153 \\
3.69 & 0.56 & 1.11 & 111 \\
4.08 & 0.50 & 1.11 & 146 \\
\hline
\end{tabular}

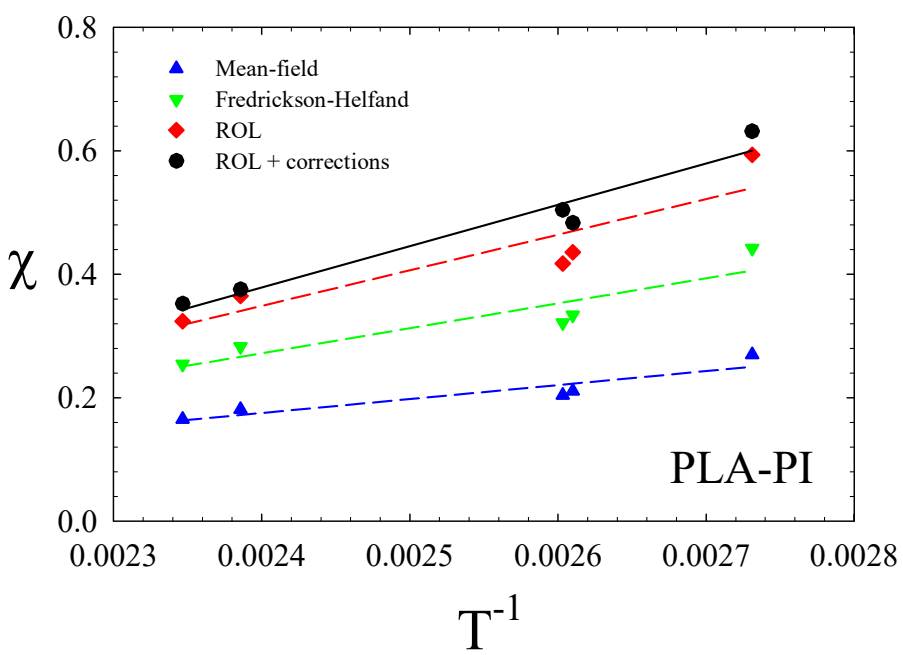

Fits to $\chi(T)=A / T+B$ for PLA-PI

\begin{tabular}{lcc}
\hline $\begin{array}{l}\text { Prediction for } \\
(\chi N)_{\text {ODT }}\end{array}$ & $A$ & $B \times 10^{3}$ \\
\hline Mean-field & 226 & -368 \\
Fredrickson-Helfand & 404 & -697 \\
ROL & 577 & -1034 \\
ROL + corrections & 669 & -1227 \\
\hline
\end{tabular}




\section{Poly(ethyleneoxide)-Poly(ethylenepropylene)}

\begin{tabular}{cccc}
\multicolumn{4}{c}{ PEO-PEP diblock polymers } \\
\hline $\begin{array}{c}M_{n} \\
\mathrm{~kg} / \mathrm{mol})\end{array}$ & $f_{\text {PEO }}$ & $Đ$ & $\begin{array}{c}T_{\mathrm{ODT}} \\
\left({ }^{\circ} \mathrm{C}\right)\end{array}$ \\
\hline 2.2 & 0.52 & 1.11 & 77 \\
3.0 & 0.51 & 1.08 & 156 \\
\hline
\end{tabular}

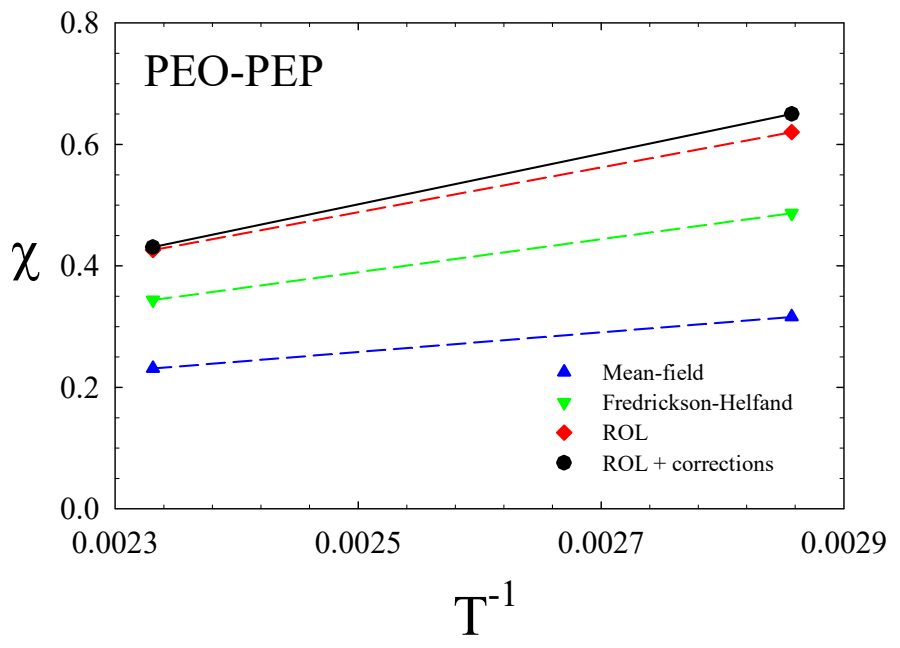

Fits to $\chi(T)=A / T+B$ for PEO-PEP

\begin{tabular}{|c|c|c|}
\hline $\begin{array}{l}\text { Prediction } \\
(\chi N)_{\mathrm{ODT}}\end{array}$ & $A$ & $B \times 10^{3}$ \\
\hline Mean-field & 162 & -145 \\
\hline Fredrickson-Helfand & 272 & -289 \\
\hline ROL & 369 & -434 \\
\hline ROL + corrections & 416 & -540 \\
\hline
\end{tabular}




\section{Poly(ethyleneoxide)-Poly(ethylethylene)}

\begin{tabular}{cccc}
\multicolumn{4}{c}{ PEO-PEE diblock polymers } \\
\hline $\begin{array}{c}M_{n} \\
(\mathrm{~kg} / \mathrm{mol})\end{array}$ & $f_{\text {PEO }}$ & $Đ$ & $\begin{array}{c}T_{\text {ODT }} \\
\left({ }^{\circ} \mathrm{C}\right)\end{array}$ \\
\hline 2.2 & 0.52 & 1.11 & 64 \\
3.6 & 0.48 & 1.12 & 177 \\
\hline
\end{tabular}

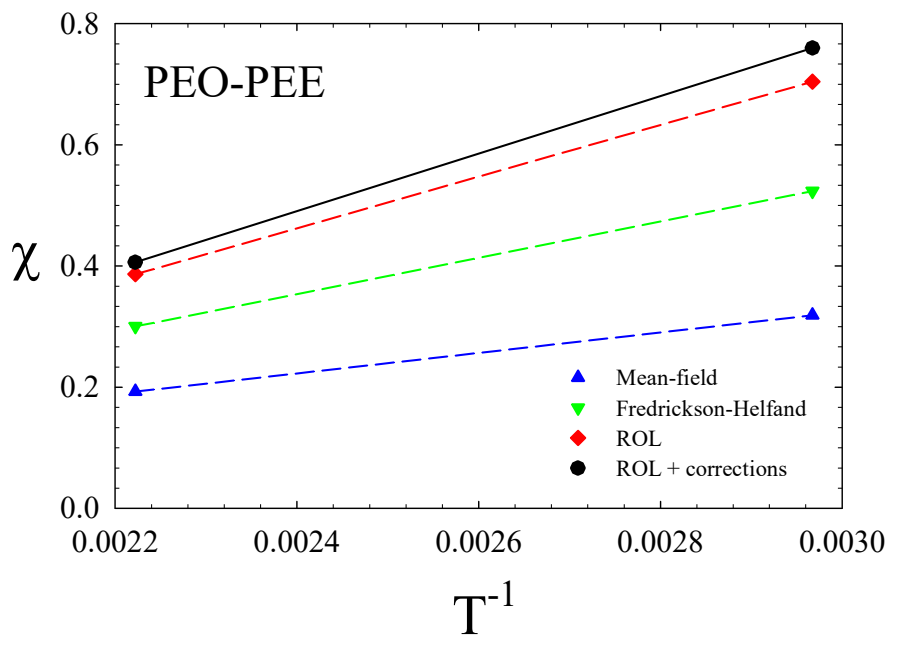

Fits to $\chi(T)=A / T+B$ for PEO-PEE

\begin{tabular}{lcc}
\hline $\begin{array}{l}\text { Prediction for } \\
(\chi N)_{\text {ODT }}\end{array}$ & $A$ & $B \times 10^{3}$ \\
\hline Mean-field & 169 & -183 \\
Fredrickson-Helfand & 300 & -366 \\
ROL & 426 & -560 \\
ROL + corrections & 475 & -649 \\
\hline
\end{tabular}




\section{Appendix B}

\section{Random Phase Approximation}

The random phase approximation was first applied to polymers by de Gennes [1]. It is an approximation to the mean-field theory of polymers (SCFT) that involves expanding the partition function in powers of fluctuations around a homogeneous state. This is called the weak inhomogeneity expansion. This method was applied to monodisperses diblocks by Leibler [12]. The fields $w_{A}$ and $w_{B}$, are expanded around the homogeneous fields $W_{A 0}$ and $W_{B 0}$, the rest of this section scales $s$ so it runs between 0 and 1

$$
W(\vec{r}, s)= \begin{cases}w_{A 0}+\xi \Delta w_{A}(\vec{r}) & 0 \leq s \leq f \\ w_{B 0}+\xi \Delta w_{B}(\vec{r}) & f<s \leq 1\end{cases}
$$

where $\xi$ is a arbitrary, small number representing the strength of the fluctuations of the field. By limiting the forward propagator to only the chain between $0 \leq s \leq N f$, and the backwards propagator between $N f<s \leq N$, the propagators are only influenced by one of the fields each. When $\xi$ is small, around zero, the field is constant and the forwards and backwards polymer propagators can be solved for from the propagator equations,

$$
\begin{gathered}
\frac{d q(\vec{r}, s)}{d s}=\frac{a^{2} N}{6} \nabla^{2} q(\vec{r}, s)-w_{A 0} q(\vec{r}, s) \\
\frac{d q^{\dagger}(\vec{r}, s)}{d s}=-\frac{a^{2} N}{6} \nabla^{2} q(\vec{r}, s)^{\dagger}+w_{B 0} q(\vec{r}, s)^{\dagger} .
\end{gathered}
$$

The solution to this starting from a uniform initial conditions is simply to have the propagators constant in space and vary in chain length. This gives $q(\vec{r}, s)=q(\overrightarrow{0}, 0) e^{-w_{A 0} s}$ and $q^{\dagger}(\vec{r}, s)=q^{\dagger}(\overrightarrow{0}, 0) e^{w_{B 0} s}$. Now, to solve for the propagators with the fields allowed to 
fluctuate, a multiplicative correction to the constant field propagators is added: $q(\vec{r}, s)=$ $e^{-w_{A 0} s} \Delta q(\vec{r}, s)$ and $q^{\dagger}(\vec{r}, s)=e^{w_{B 0} s} \Delta q^{\dagger}(\vec{r}, s)$. This is subbed into the differential equation for the propagators and simplified to get

$$
\frac{d \Delta q(\vec{r}, s)}{d s}=\frac{a^{2} N}{6} \nabla^{2} \Delta q(\vec{r}, s)-\xi \Delta w_{A}(\vec{r}) \Delta q(\vec{r}, s)
$$

and

$$
\frac{d \Delta q^{\dagger}(\vec{r}, s)}{d s}=-\frac{a^{2} N}{6} \nabla^{2} \Delta q^{\dagger}(\vec{r}, s)+\xi \Delta w_{B}(\vec{r}) \Delta q^{\dagger}(\vec{r}, s) .
$$

Now, to solve the above equations for small $\xi$, perturbation methods can be used. This works by expanding $\Delta q(\vec{r}, s)$ in terms of $\xi$ to get $\Delta q(\vec{r}, s)=1+\sum_{i=1}^{\infty} c^{(i)}(\vec{r}, s) \xi^{i}$. Similarly, $\Delta q^{\dagger}(\vec{r}, s)=1+\sum_{i=1}^{\infty}\left(c^{\dagger}(\vec{r}, s)\right)^{(i)} \xi^{i}$. Subbing these in and isolating by powers of $\xi$ gives

$$
0=\sum_{i=1}^{\infty}\left[\frac{c^{(i)}(\vec{r}, s)}{d s}-\frac{a^{2} N}{6} \nabla^{2} c^{(i)}(\vec{r}, s)+\Delta w_{A}(\vec{r}) c^{(i-1)}(\vec{r}, s)\right] \xi^{i}
$$

and

$$
0=\sum_{i=1}^{\infty}\left[\frac{d\left(c^{\dagger}\right)^{(i)}(\vec{r}, s)}{d s}+\frac{a^{2} N}{6} \nabla^{2}\left(c^{\dagger}\right)^{(i)}(\vec{r}, s)-\Delta w_{B}(\vec{r})\left(c^{\dagger}\right)^{(i-1)}(\vec{r}, s)\right] \xi^{i} .
$$

For this equation to be true for all values of $\xi$, it must be true for each order of $\xi$. This gives

$$
0=\frac{d c^{(i)}(\vec{r}, s)}{d s}-\frac{a^{2} N}{6} \nabla^{2} c^{(i)}(\vec{r}, s)+\Delta w_{A}(\vec{r}) c^{(i-1)}(\vec{r}, s)
$$

and

$$
0=\frac{d\left(c^{\dagger}\right)^{(i)}(\vec{r}, s)}{d s}+\frac{a^{2} N}{6} \nabla^{2}\left(c^{\dagger}\right)^{(i)}(\vec{r}, s)-\Delta w_{B}(\vec{r})\left(c^{\dagger}\right)^{(i-1)}(\vec{r}, s) .
$$

By Fourier transforming these equations, we can solve for the transformed quantities $\left(c^{*}\right)^{(i)}(\vec{k}, s)$ and $\left(c^{* \dagger}\right)^{(i)}(\vec{k}, s)$ in terms of the fields $\Delta w_{A}(\vec{k})$ and $\Delta w_{B}(\vec{k})$. Doing this for $i=1$ gives

$$
\left(c^{*}\right)^{(1)}(\vec{k}, s)=\frac{6}{a^{2} N k^{2}}\left(1-e^{\frac{-a^{2} k^{2}}{6} N s}\right) \Delta w_{A}(\vec{k})
$$

and

$$
\left(c^{* \dagger}\right)^{(1)}(\vec{k}, s)=\frac{6}{a^{2} N k^{2}}\left(1-e^{\frac{a^{2} k^{2}}{6} N s}\right) \Delta w_{B}(\vec{k}) .
$$

Solving for $i=2$ we have 
$\left(c^{*}\right)^{(2)}(\vec{k}, s)=\sum_{\overrightarrow{k^{\prime}}} \frac{36 \Delta w_{A}\left(\overrightarrow{k^{\prime}}\right) \Delta w_{A}\left(\vec{k}-\overrightarrow{k^{\prime}}\right)}{a^{4} N^{2}|\vec{k}|^{2}\left|\vec{k}-\overrightarrow{k^{\prime}}\right|^{2}}\left[1-e^{-\frac{a^{2} k^{2}}{6} N s}-\frac{k^{2}}{|\vec{k}|^{2}-\left|\vec{k}-\overrightarrow{k^{\prime}}\right|^{2}}\left(e^{-\frac{a^{2}\left|\vec{k}-\vec{k}^{\prime}\right|^{2}}{6} N s}-e^{-\frac{a^{2} k^{2}}{6} N s}\right)\right]$

and

$\left(c^{* \dagger}\right)^{(2)}(\vec{k}, s)=\sum_{\overrightarrow{k^{\prime}}} \frac{36 \Delta w_{B}\left(\overrightarrow{k^{\prime}}\right) \Delta w_{B}\left(\vec{k}-\overrightarrow{k^{\prime}}\right)}{a^{4} N^{2}|\vec{k}|^{2}\left|\vec{k}-\overrightarrow{k^{\prime}}\right|^{2}}\left[1-e^{\frac{a^{2} k^{2}}{6} N s}-\frac{k^{2}}{|\vec{k}|^{2}-\left|\vec{k}-\overrightarrow{k^{\prime}}\right|^{2}}\left(e^{\frac{a^{2}\left|\vec{k}-\vec{k}^{\prime}\right|^{2}}{6} N s}-e^{\frac{a^{2} k^{2}}{6} N s}\right)\right]$.

Then, simplifying $\left(c^{*}\right)^{(2)}(\overrightarrow{0}, s)$ and $\left(c^{* \dagger}\right)^{(2)}(\overrightarrow{0}, s)$ with the definition of the Deybe function, $g_{1}(x, f)=2\left[f x+e^{-f x}-1\right] / x^{2}$, we get the following form for the corrections,

$$
\left(c^{*}\right)^{(2)}(\overrightarrow{0}, s)=\sum_{\overrightarrow{k^{\prime}}} \frac{N^{2} s^{2}}{2} g_{1}\left(\frac{N a^{2}\left|\overrightarrow{k^{\prime}}\right|^{2}}{6}, s\right) \Delta w_{A}\left(\overrightarrow{k^{\prime}}\right) \Delta w_{A}\left(-\overrightarrow{k^{\prime}}\right)
$$

and

$$
\left(c^{* \dagger}\right)^{(2)}(\overrightarrow{0}, s)=\sum_{\overrightarrow{k^{\prime}}} \frac{N^{2} s^{2}}{2} g_{1}\left(\frac{N a^{2}\left|\overrightarrow{k^{\prime}}\right|^{2}}{6}, s\right) \Delta w_{B}\left(\overrightarrow{k^{\prime}}\right) \Delta w_{B}\left(-\overrightarrow{k^{\prime}}\right) .
$$

Subbing this into the definition of the partition function $Q=\int_{\mathbb{R}^{3}} d^{3} \vec{r} q^{\dagger}(\vec{r}, N-N f) q(\vec{r}, N f)$ inverting the Fourier transform and using the identity for Fourier transforms $f^{*}(\overrightarrow{0})=$ $\int_{\mathbb{R}^{3}} d^{3} \vec{r} f(\vec{r})$ to solve the integral for $Q$ gives

$Q=Q_{0}\left[1+\sum_{\vec{k}}\left(\left(c^{\dagger}\right)^{(1)}(\vec{k}, f)(c)^{(1)}(\vec{k}, 1-f)\right) \xi^{2}+\left(c^{* \dagger}\right)^{(2)}(\overrightarrow{0}, 1-f) \xi^{2}+\left(c^{*}\right)^{(2)}(\overrightarrow{0}, f) \xi^{2}+O\left(\xi^{3}\right)\right]$,

where $Q_{0}$ is the partition function in the constant fields. To write a simplified partition function $Q[\overrightarrow{\Delta w}]$ we make the following definitions using vector notation:

$$
\begin{gathered}
\overrightarrow{\Delta w}(\vec{k})=\left(\begin{array}{c}
\Delta w_{A}(\vec{k}) \\
\Delta w_{B}(\vec{k})
\end{array}\right), \\
s_{A, A}(k, f)=N g_{1}\left(\frac{N a^{2} k^{2}}{6}, f\right), \\
s_{B, B}(k, f)=N g_{1}\left(x \frac{N a^{2} k^{2}}{6}, 1-f\right),
\end{gathered}
$$




$$
s_{A, B}(k, f)=s_{B, A}(k, 1-f)=\frac{N}{2}\left[g_{1}\left(\frac{N a^{2} k^{2}}{6}, 1\right)-g_{1}\left(\frac{N a^{2} k^{2}}{6}, f\right)-g_{1}\left(\frac{N a^{2} k^{2}}{6}, 1-f\right)\right],
$$

and

$$
S(k, f)=\left(\begin{array}{ll}
s_{A, A}(k, f) & s_{A, B}(k, f) \\
s_{B, A}(k, f) & s_{B, B}(k, f)
\end{array}\right) .
$$

This gives the simplified expression for the partition function,

$$
Q[\overrightarrow{\Delta w}]=Q_{0}\left[1+\frac{\xi^{2}}{2 V} \sum_{\vec{k}} \overrightarrow{\Delta w}^{T}(\vec{k}) S(\|\vec{k}\|, f) \overrightarrow{\Delta w}(\vec{k})+O\left(\xi^{3}\right)\right] .
$$

Now we need to apply the mean-field conditions to find $\phi_{A}$ and $\phi_{B}$. Starting with the relationship between $\overrightarrow{\Delta \phi}$ and $\overrightarrow{\Delta w}$,

$$
\Delta \phi_{A}(\vec{k})=-s_{A, A}(k, f) \Delta w_{A}(\vec{k})-s_{A, B}(k, f) \Delta w_{B}(\vec{k})
$$

and

$$
\Delta \phi_{B}(\vec{k})=-s_{B, A}(k, f) \Delta w_{A}(\vec{k})-s_{B, B}(k, f) \Delta w_{B}(\vec{k}) .
$$

This gives $\overrightarrow{\Delta \phi}(\vec{k})=S(k, f) \overrightarrow{\Delta w}(\vec{k})$, so then we can find $\overrightarrow{\Delta w}(\vec{k})=S(k, f)^{-1} \overrightarrow{\Delta \phi}(\vec{k})$. Plugging this result, and the mean-field equations for $\phi_{A}(\vec{k})+\phi_{B}(\vec{k})=1$ and $\chi N\left(\phi_{B}(\vec{k})-\phi_{A}(\vec{k})\right)=$ $w_{A}(\vec{k})-w_{B}(\vec{k})$, into the partition function to second-order in $\xi$ gives,

$$
Q[\overrightarrow{\Delta w}]=Q_{0}\left[1+\frac{\xi^{2}}{2 V} \sum_{\vec{k}} S^{-1}(\|\vec{k}\|) \Delta \phi_{A}(\vec{k}) \Delta \phi_{A}(-\vec{k})\right] .
$$

where $S(k)$ is the structure funtion for a disordered melt

$$
S(k)=\frac{W(k)}{s(k)-2 \chi W(k)} .
$$

For simplicity, we define $\operatorname{Det}(S(k, f))=W(k)$ and $s(k)=s_{B, B}(|\vec{k}|, f)+s_{A, A}(k, f)+$ $2 s_{B, A}(k, f)$. Equation B.24 can be simplified by substituting $F(k)=N W(k) / s(k)$ to give

$$
S(k)=\frac{N}{F(k)-2 \chi N} .
$$

This gives the scattering function for monodispers diblock copolymers. Later the processes was generalized for polydisperse melts by Hong and Noolandi in 1984 [7]. This 
change results in replacing the Debye function $g_{1}(f, x)$ by the number-averaged Debye function

$$
\bar{g}_{1}(\bar{f}, \bar{x})=2\left[\bar{f} \bar{x}+(1+\bar{f} \bar{x} K)^{-K^{-1}}-1\right] / \bar{x}^{2},
$$

for Equations B.17, B.18, and B.19. The over bars represent number averages so $\bar{x}=$ $k^{2} a^{2}<N>_{N} / 6$ and $\bar{f}=\langle f\rangle_{N}$. The polydispersity index is related to $K=\frac{\langle N\rangle_{n}}{\langle N\rangle_{w}}-1$ by $K=\frac{1}{\mathrm{Ð}-1}$. With this, we have a polydisperse scattering function for diblock copolymers. However it can be simplified significantly for $\bar{f}=0.5$. In this case, $s_{A, A}(k)=s_{B, B}(k)$ so the function $F(k)$ can be written as

$$
F(k)=N \frac{s_{A, A}^{2}(k)-s_{A, B}^{2}(k)}{2 s_{A, A}(k)+2 s_{A, B}(k)} .
$$

Rewriting the numerator using difference of squares and canceling the $s_{A, A}(k)+s_{A, B}(k)$ factor gives

$$
F(k)=N \frac{s_{A, A}(k)-s_{A, B}(k)}{2} .
$$

Now, subbing in $s_{A, A}(k)$ and $s_{A, B}(k)$,

$$
F(k)=\frac{\bar{g}_{1}(0.5, x)-\frac{1}{2}\left[\bar{g}_{1}(1, x)-2 \bar{g}_{1}(0.5, x)\right]}{2} .
$$

Simplifying further gives the final simple form

$$
F(k)=\bar{g}_{1}(0.5, x)-\frac{1}{4} \bar{g}_{1}(1, x) .
$$

